\title{
Quinine Synthesis Studies: A Radical-Ionic Annulation via Mn-Mediated Addition to Chiral $\mathbf{N}$-Acylhydrazones
}

\author{
Chandra Sekhar Korapala, Jun Qin, and Gregory K. Friestad*
}

Department of Chemistry, University of Iowa, Iowa City, Iowa, 52242

gregory-friestad@uiowa.edu

\section{Supporting Information}

\author{
Table of Contents \\ Procedures and Characterization Data for New Compounds \\ pp. S1-S14 \\ NMR Spectra for Compounds 2, 3a-3d, 8d, 10-12, 16, 18. \\ pp. $\mathrm{S} 15-\mathrm{S} 27$ \\ COSY and NOESY Spectra of Compound $\mathbf{1 8 .}$ \\ pp. S28-S30 \\ NMR Spectra for Compounds S4, S6, S7, S7', S11. \\ pp. S31-S35 \\ Crystallographic Data for Compound $\mathbf{S 9}$. \\ pp. S36-S38
}

Materials and Methods. Reactions employed oven- or flame-dried glassware under nitrogen unless otherwise noted. THF, diethyl ether, benzene and toluene were distilled from sodium/benzophenone ketyl under argon. $\mathrm{CH}_{2} \mathrm{Cl}_{2}$ was distilled from $\mathrm{CaH}_{2}$ under argon or nitrogen. Alternatively, these solvents were purchased inhibitor-free and were sparged with argon and passed through columns of activated alumina prior to use (dropwise addition of blue benzophenone ketyl solution revealed the THF purified in this manner sustained the blue color more readily than the control sample purified by distillation). Nitrogen was passed successively through columns of anhydrous $\mathrm{CaSO}_{4}$ and $\mathrm{R} 3-11$ catalyst for removal of water and oxygen, respectively. All other materials were used as received from commercial sources unless otherwise noted. Thin layer chromatography (TLC) employed glass $0.25 \mathrm{~mm}$ silica gel plates with UV indicator. Flash chromatography columns were packed with 230-400 mesh silica gel as a slurry in the initial elution solvent. Gradient flash chromatography was conducted by adsorption of product mixtures on silica gel, packing over a short pad of clean silica gel as a slurry in hexane, and eluting with a continuous gradient from hexane to the indicated solvent. Radial chromatography refers to centrifugally accelerated thin-layer chromatography performed with a Chromatotron using commercially supplied rotors. Melting points are uncorrected. Nuclear magnetic resonance (NMR) data were obtained at operating frequencies indicated in the text, and are reported in units of ppm. Infrared spectra were recorded using a single beam FT-IR spectrophotometer by standard transmission methods or by use of an attenuated total reflectance (ATR) probe. Optical rotations were determined using a digital polarimeter operating at ambient 
temperature. Low resolution mass spectra were obtained using sample introduction by dip, liquid chromatography or gas chromatography. High resolution mass spectra and combustion analyses were obtained from external commercial and institutional services. Chromatographic diastereomer ratio analyses employed GCMS with $15 \mathrm{~m}$ x $0.25 \mathrm{~mm}$ x $0.25 \mu \mathrm{m}$ (1 x i.d. x f.t.) $5 \%$ phenyl-95\%-dimethylsiloxane column and helium as mobile phase or HPLC with Microsorb-MV Si 8um 100A or Chiralcel OD columns (2-propanol/hexane as mobile phase) or Chirex 3014 column (chloroform/hexane as mobile phase).

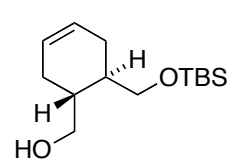

((1R,6R)-6-((tert-butyldimethylsilyloxy)methyl)cyclohex-3-enyl)methanol $((-)-2)$. To a solution of $\left((1 R, 6 R)-6\right.$-(hydroxymethyl)cyclohex-3-enyl)methanol ${ }^{1}$ $(891 \mathrm{mg}, 6.28 \mathrm{mmol})$ in THF (40 mL) was added $n$-BuLi (2 M in hexane, 3.50 $\mathrm{mL}, 7.0 \mathrm{mmol}$ ) at $0^{\circ} \mathrm{C}$. After warming to room temperature for 80 min under $\mathrm{N}_{2}$, TBSCl (947 mg, $6.28 \mathrm{mmol}$ ) was added and the mixture was stirred overnight. The reaction was partitioned between EtOAc and saturated aqueous $\mathrm{NaHCO}_{3}$. The aqueous layer was extracted with $\mathrm{CH}_{2} \mathrm{Cl}_{2}$ (3 times). The combined organic phase was dried $\left(\mathrm{Na}_{2} \mathrm{SO}_{4}\right)$, concentrated and purified by flash chromatography (hexane/EtOAc 7:1 to 1:1) to afford alcohol (-)-2 (1.60 g, 99\% yield) as a colorless oil. $[\alpha]_{\mathrm{D}}^{30}-30.2\left(c 4.6, \mathrm{CHCl}_{3}\right)$; IR (film) 3371, 3024, 2955, 2929, 2899, 2857, 1472, 1436, 1361, 1256, 1102, $998 \mathrm{~cm}^{-1} ;{ }^{1} \mathrm{H}$ NMR (500 MHz, $\left.\mathrm{CDCl}_{3}\right) \delta$ 5.75-5.55 (m, 2H), 3.80-3.43 (m, 4H), 3.42-2.87 (br s, 1H), 2.06-1.88 (m, 3H), 1.82-1.56 (m, 3H), $0.90(\mathrm{~s}, 9 \mathrm{H}), 0.08$ $(\mathrm{s}, 6 \mathrm{H}) ;{ }^{13} \mathrm{C} \mathrm{NMR}\left(125 \mathrm{MHz}, \mathrm{CDCl}_{3}\right) \delta 126.6,125.6,67.0,65.7,40.5,39.1,28.7,28.5,25.8$, 18.1, -5.6, -5.5; MS (CI) $\mathrm{m} / z$ (relative intensity) $257\left([\mathrm{M}+\mathrm{H}]^{+}, 66 \%\right), 107(100 \%)$. The antipode $(+)-2$ was prepared by the same procedure from $((1 S, 6 S)$-6-(hydroxymethyl)cyclohex-3enyl)methanol in $93 \%$ yield; $[\alpha]_{\mathrm{D}}{ }^{21}+31.6\left(c 3.22, \mathrm{CHCl}_{3}\right)$.

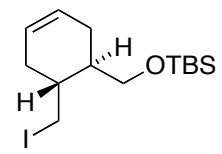

$((1 R, 6 R)-6-(($ tert-butyldimethylsilyloxy)methyl)cyclohex-3-enyl)methanol ((-)-3a). To a solution of alcohol (-)-2 (150 mg, $0.59 \mathrm{mmol})$ in $\mathrm{CH}_{2} \mathrm{Cl}_{2}$ (6 mL) was added imidazole (100 mg, $1.46 \mathrm{mmol})$ and $\mathrm{PPh}_{3}(309 \mathrm{mg}, 1.18 \mathrm{mmol})$. After $10 \mathrm{~min}, \mathrm{I}_{2}(300 \mathrm{mg}, 1.18 \mathrm{mmol})$ was added in two portions over $10 \mathrm{~min}$. After $15 \mathrm{~min}$, the reaction mixture was partitioned between $\mathrm{CH}_{2} \mathrm{Cl}_{2}$ and saturated aqueous $\mathrm{Na}_{2} \mathrm{~S}_{2} \mathrm{O}_{3}$. The organic phase was dried $\left(\mathrm{Na}_{2} \mathrm{SO}_{4}\right)$, concentrated and purified by flash chromatography (hexane/EtOAc $10: 1$ to $3: 1)$ to afford iodide (-)-3a as a colorless liquid (186 mg, 86\% yield). $[\alpha]_{\mathrm{D}}{ }^{27}-95.0$ (c 4.4, $\mathrm{CHCl}_{3}$ ); IR (film) 3025, 2954, 2928, 2897, 2856, 1471, 1462, 1256, 1107, $982 \mathrm{~cm}^{-1}$; ${ }^{1} \mathrm{H}$ NMR $\left(500 \mathrm{MHz}, \mathrm{CDCl}_{3}\right) \delta 5.68-5.54(\mathrm{~m}, 2 \mathrm{H}), 3.67(\mathrm{dd}, J=9.8,4.1 \mathrm{~Hz}, 1 \mathrm{H}), 3.54(\mathrm{dd}, J=9.8,5.3 \mathrm{~Hz}$, $1 \mathrm{H}), 3.39$ (dd, $J=9.8,6.4 \mathrm{~Hz}, 1 \mathrm{H}), 3.32(\mathrm{dd}, J=9.8,6.4 \mathrm{~Hz}, 1 \mathrm{H}), 2.18-1.92(\mathrm{~m}, 4 \mathrm{H}), 1.76-1.66$ $(\mathrm{m}, 1 \mathrm{H}), 1.66-1.56(\mathrm{~m}, 1 \mathrm{H}), 0.90(\mathrm{~s}, 9 \mathrm{H}), 0.05(\mathrm{~d}, J=2.7 \mathrm{~Hz}, 6 \mathrm{H}) ;{ }^{13} \mathrm{C}$ NMR $\left(125 \mathrm{MHz}, \mathrm{CDCl}_{3}\right)$ $\delta$ 126.1, 125.0, 64.2 , 39.6, 35.2, 31.1, 27.0, 25.9, 18.2, 14.9, -5.5; MS (CI) $\mathrm{m} / \mathrm{z}$ (relative intensity) $367\left([\mathrm{M}+\mathrm{H}]^{+}, 26 \%\right), 239(80 \%), 107(100 \%)$. The antipode (+)-3a was prepared by the same procedure from $(+)-2$ in $81 \%$ yield; $[\alpha]_{\mathrm{D}}^{21}+54\left(c 5.1, \mathrm{CHCl}_{3}\right)$.

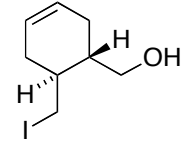

((1S,6S)-6-(iodomethyl)cyclohex-3-enyl)methanol (3b). A solution of (+)-3a (160 $\mathrm{mg}, 0.43 \mathrm{mmol})$ and camphorsulfonic acid $(9 \mathrm{mg}, 0.04 \mathrm{mmol})$ in methanol (3 $\mathrm{mL}$ ) was stirred for $4 \mathrm{~h}$ at room temperature. Concentration and flash chromatography (hexane/EtOAc 20:1 to 2:1) yielded $\mathbf{3 b}$ (92 $\mathrm{mg}, 85 \%$ yield) as a colorless oil; $[\alpha]_{\mathrm{D}}{ }^{23}+48.28\left(c 1.11, \mathrm{CHCl}_{3}\right) ; 3553,3022,2895,2837,1432,1182,1061,1034 \mathrm{~cm}^{-1} ;{ }^{1} \mathrm{H} \mathrm{NMR}$ $\left(300 \mathrm{MHz}, \mathrm{CDCl}_{3}\right) \delta$ 5.65-5.55 (m, 2H), 3.74-3.62 (m, 2H), 3.45-3.35 (m, 2H), 2.87 (br s, 1H), 2.16-1.92 (m, 4H), 1.85-1.77 (m, 1H), 1.67-1.61 (m, 1H); $\left.{ }^{13} \mathrm{C} \mathrm{NMR} \mathrm{(75} \mathrm{MHz,} \mathrm{CDCl}_{3}\right) \delta 125.6$, 
125.2, 64.9, 39.4, 35.4, 30.7, 26.5, 14.8; MS (EI) $\mathrm{m} / z$ (relative intensity) $252\left(\mathrm{M}^{+}, 1 \%\right), 234$ $\left(\left[\mathrm{M}-\mathrm{H}_{2} \mathrm{O}\right]^{+}, 4 \%\right), 125\left([\mathrm{M}-\mathrm{I}]^{+}, 20 \%\right)$.

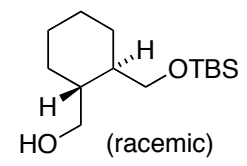

( \pm )-trans-(2-((tert-butyldimethylsilyloxy)methyl)cyclohexyl)methanol $\left(( \pm)-\right.$ S1). To a solution of $( \pm)$-trans-2-(hydroxymethyl)cyclohexylmethanol ${ }^{2}$ (533 $\mathrm{mg}, 3.69 \mathrm{mmol})$ in THF $(16 \mathrm{~mL})$ was added $n$-BuLi $(1.6 \mathrm{M}$ in hexane, $2.4 \mathrm{~mL}$, $3.87 \mathrm{mmol}$ ) at $0{ }^{\circ} \mathrm{C}$. After warming to room temperature for 80 min under $\mathrm{N}_{2}$, tert-butyldimethylsilyl chloride $(556 \mathrm{mg}, 3.69 \mathrm{mmol}$ ) was added. After ca. $12 \mathrm{~h}$, the reaction was partitioned between EtOAc and saturated aqueous $\mathrm{NaHCO}_{3}$. The aqueous phase was extracted with $\mathrm{CH}_{2} \mathrm{Cl}_{2}(3 \times 5 \mathrm{~mL})$. The combined organic phase was dried $\left(\mathrm{Na}_{2} \mathrm{SO}_{4}\right)$ and concentrated. Flash chromatography (hexane/EtOAc 10:1 to 2:1) afforded alcohol ( \pm )-S1 ${ }^{3}$ (955 $\mathrm{mg},>99 \%$ yield) as a colorless oil; IR (film) 3362, 2927, 2856, 1471, 1388, 1255, 1110, 1066, $1005 \mathrm{~cm}^{-1}$; ${ }^{1} \mathrm{H}$ NMR $\left(300 \mathrm{MHz}, \mathrm{CDCl}_{3}\right) \delta 3.78$ (br s, 1H), 3.51-3.35 (m, 4H), 1.69-1.65 (m, 2H), 1.59-1.51 (m, 2H), 1.26-0.90 (m, 6H), $0.84(\mathrm{~s}, 9 \mathrm{H}), 0.02(\mathrm{~s}, 6 \mathrm{H}) ;{ }^{13} \mathrm{C} \mathrm{NMR}\left(75 \mathrm{MHz}, \mathrm{CDCl}_{3}\right) \delta$ $68.6,67.4,45.5,44.1,30.2,29.9,26.28,26.26,25.9,18.3,-5.3,-5.4$; Anal. Calcd for $\mathrm{C}_{14} \mathrm{H}_{30} \mathrm{O}_{2} \mathrm{Si}: \mathrm{C}, 65.06 ; \mathrm{H}, 11.70$. Found: C, 65.07; H, 11.80 .

tert-butyl((2-(iodomethyl)cyclohexyl)methoxy)dimethylsilane (( \pm$)-\mathrm{S} 2)$. To

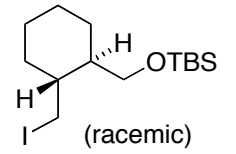
a solution of alcohol $( \pm)-\mathrm{S1}(955 \mathrm{mg}, 3.69 \mathrm{mmol})$ in $\mathrm{CH}_{2} \mathrm{Cl}_{2}$ was added imidazole $(628 \mathrm{mg}, 9.22 \mathrm{mmol})$ and triphenylphosphine $(1.94 \mathrm{~g}, 7.39 \mathrm{mmol})$. After $10 \mathrm{~min}$, iodine $(1.87 \mathrm{~g}, 7.39 \mathrm{mmol})$ was added in two portions over $10 \mathrm{~min}$. After $15 \mathrm{~min}$, the reaction mixture was partitioned between $\mathrm{CH}_{2} \mathrm{Cl}_{2}$ and saturated aqueous $\mathrm{Na}_{2} \mathrm{~S}_{2} \mathrm{O}_{3}$. The organic phase was dried $\left(\mathrm{Na}_{2} \mathrm{SO}_{4}\right)$, concentrated and purified by gradient flash chromatography (hexane to 10:1 hexane/EtOAc) to afford iodide ( \pm )-S2 (972 mg, 72\% yield) as a colorless oil; IR (film) 2953, 2925, 2854, 1470, 1386, 1255, 1107, $1003 \mathrm{~cm}^{-1} ;{ }^{1} \mathrm{H}$ NMR (300 MHz, CDCl 3 ) $\delta .59$ $(\mathrm{dd}, J=10.2,3.8 \mathrm{~Hz}, 1 \mathrm{H}), 3.48(\mathrm{dd}, J=10.1,1.9,1 \mathrm{H}), 3.39(\mathrm{dd}, J=9.8,2.5,1 \mathrm{H}), 3.29(\mathrm{dd}, J=$ 9.7, 5.4, 1H), 1.78-1.60 (m, 4H), 1.27-1.09 (m, 6H), $0.88(\mathrm{~s}, 9 \mathrm{H}), 0.03(\mathrm{~s}, 6 \mathrm{H}) ;{ }^{13} \mathrm{C} \mathrm{NMR}(75$ $\mathrm{MHz}_{\mathrm{CDCl}}$ ) $\delta$ 65.3, 44.0, 39.3, 33.4, 29.5, 26.3, 26.1, 25.9, 18.5, 17.1, -5.24, -5.25; MS (EI) $\mathrm{m} / z$ (relative intensity) $311\left([\mathrm{M}-t-\mathrm{Bu}]^{+}, 93 \%\right), 241\left([\mathrm{M}-\mathrm{I}]^{+}, 10 \%\right)$; Anal. Calcd for $\mathrm{C}_{14} \mathrm{H}_{29} \mathrm{IOSi}: \mathrm{C}$, 45.65; H, 7.94. Found: C, 45.91; H, 8.13.

(2-(iodomethyl)cyclohexyl)methanol (( \pm$)-3 c)$. A solution of $( \pm)-\mathbf{S 2}$ (410 $\mathrm{mg}$,

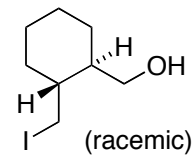
$1.12 \mathrm{mmol})$ and camphorsulfonic acid (30 mg, $0.1 \mathrm{mmol})$ in methanol $(5 \mathrm{~mL})$ was stirred for $4 \mathrm{~h}$ at room temperature. Concentration and flash chromatography (hexane/EtOAc 9:1 to 2:1) yielded ( \pm )-3c (265 mg, 93\% yield) as a colorless oil; IR (film) 3338, 2921, 2852, 1446, 1292, 1240, 1183, $1083 \mathrm{~cm}^{-1} ;{ }^{1} \mathrm{H}$ NMR $\left(300 \mathrm{MHz}, \mathrm{CDCl}_{3}\right) \delta$ 3.59-3.49 (m, 2H), 3.35-3.26 (m, 2H), $3.19(\mathrm{~s}, 1 \mathrm{H}), 1.75-1.60(\mathrm{~m}, 4 \mathrm{H}), 1.29-1.10(\mathrm{~m}, 5 \mathrm{H}), 1.10-$ $0.95(\mathrm{~m}, 1 \mathrm{H}) ;{ }^{13} \mathrm{C} \mathrm{NMR}\left(75 \mathrm{MHz}, \mathrm{CDCl}_{3}\right) \delta 65.1,43.6,39.0,33.2,29.0,25.9,25.6,16.9 ; \mathrm{MS}$ (EI) $\mathrm{m} / z$ (relative intensity) $254\left(\mathrm{M}^{+}, 1 \%\right), 127\left([\mathrm{M}-\mathrm{I}]^{+}, 38 \%\right)$.

2-((1R,6S)-6-((tert-Butyldimethylsilyloxy)methyl)cyclohex-3-enyl)aceto-

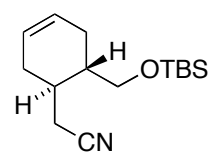

nitrile (4). A solution of (+)-3a (1.409 g, $3.85 \mathrm{mmol})$ and potassium cyanide (501 $\mathrm{mg}, 7.7 \mathrm{mmol})$ in dimethyl sulfoxide $(25 \mathrm{~mL})$ was heated at $40{ }^{\circ} \mathrm{C}$ for ca. $12 \mathrm{~h}$.

The reaction mixture was partitioned between water and diethyl ether. The organic phase was washed successively with brine and water, dried $\left(\mathrm{Na}_{2} \mathrm{SO}_{4}\right)$, and concentrated. Flash chromatography (hexane/EtOAc 20:1 to 9:1) furnished 4 (980 $\mathrm{mg}, 96 \%$ yield) as a 
colorless oil; $[\alpha]_{\mathrm{D}}{ }^{25}+60.5$ (c 1.55, $\mathrm{CHCl}_{3}$ ); IR (film) 3027, 2954, 2927, 2856, 2238, 1411, 1437 , $1254 \mathrm{~cm}^{-1}$; ${ }^{1} \mathrm{H}$ NMR $\left(300 \mathrm{MHz}, \mathrm{CDCl}_{3}\right) \delta 5.67-5.58(\mathrm{~m}, 2 \mathrm{H}), 3.65(\mathrm{dd}, J=10.4,4.7 \mathrm{~Hz}, 1 \mathrm{H})$, $3.51(\mathrm{dd}, J=10.4,5.1 \mathrm{~Hz}, 1 \mathrm{H}), 2.48(\mathrm{~d}, J=5.00 \mathrm{~Hz}, 2 \mathrm{H}), 2.28-2.21(\mathrm{~m}, 1 \mathrm{H}), 2.10-1.95(\mathrm{~m}, 4 \mathrm{H})$, $1.80-1.71(\mathrm{~m}, 1 \mathrm{H}), 0.88(\mathrm{~s}, 9 \mathrm{H}), 0.04(\mathrm{~s}, 6 \mathrm{H}) ;{ }^{13} \mathrm{C} \mathrm{NMR}\left(75 \mathrm{MHz}, \mathrm{CDCl}_{3}\right) \delta 126.3,124.7,119.1$, 64.5, 38.7, 31.9, 29.9, 27.2, 25.9, 21.4, 18.3, -5.3, -5.4; MS (EI) $\mathrm{m} / z$ (relative intensity) 266 $\left([\mathrm{M}+1]^{+}, 1 \%\right)$; Anal. Calcd for $\mathrm{C}_{15} \mathrm{H}_{27} \mathrm{NOSi}$; C, 67.87; H, 10.25; N, 5.28. Found: C, 67.83; H, $10.34 ; \mathrm{N}, 5.38$.

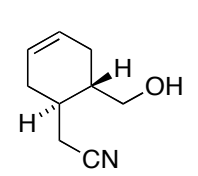

2-((1R,6S)-6-(hydroxymethyl)cyclohex-3-enyl)acetonitrile (5). A solution of $4(855 \mathrm{mg}, 3.22 \mathrm{mmol})$ and camphorsulfonic acid $(0.1 \mathrm{~g}, 0.5 \mathrm{mmol})$ in methanol (10 $\mathrm{mL}$ ) was stirred for $4 \mathrm{~h}$ at room temperature. Concentration and flash chromatography (hexane/EtOAc $10: 1$ to $1: 1)$ yielded 5 (420 $\mathrm{mg}, 85 \%$ yield) as a colorless oil; $[\alpha]_{\mathrm{D}}{ }^{25}+88.5\left(c 3.19, \mathrm{CHCl}_{3}\right)$; IR (film) 3423, 3023, 2904, 2251, 1426, $1045 \mathrm{~cm}^{-1} ;{ }^{1} \mathrm{H}$

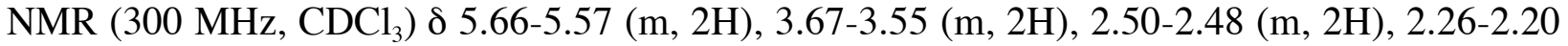
$(\mathrm{m}, 1 \mathrm{H}), 2.20-1.91(\mathrm{~m}, 5 \mathrm{H}), 1.81-1.76(\mathrm{~m}, 1 \mathrm{H}) ;{ }^{13} \mathrm{C} \mathrm{NMR}\left(75 \mathrm{MHz}, \mathrm{CDCl}_{3}\right) \delta 125.9,124.6$, 119.2, 64.5, 38.6, 31.9, 29.6, 26.7, 21.5; MS (EI) $\mathrm{m} / z$ (relative intensity) $151\left(\mathrm{M}^{+}, 1 \%\right) ; 133$ $\left(\left[\mathrm{M}-\mathrm{H}_{2} \mathrm{O}\right]^{+}, 20 \%\right)$; Anal. Calcd for $\mathrm{C}_{9} \mathrm{H}_{13} \mathrm{NO} ; \mathrm{C}, 71.49 ; \mathrm{H}, 8.67 ; \mathrm{N}, 9.26$. Found: C, 71.08; H, $8.77 ; \mathrm{N}, 8.93$.

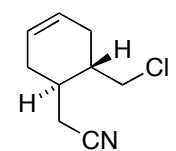

2-((1R,6S)-6-(chloromethyl)cyclohex-3-enyl)acetonitrile (6). A solution of 5 (378 $\mathrm{mg}, 2.47 \mathrm{mmol})$ and triphenylphosphine $(1.29 \mathrm{~g}, 4.94 \mathrm{mmol})$ in carbon tetrachloride $(10 \mathrm{~mL})$ was heated under reflux for $10 \mathrm{~h}$. Concentration and flash chromatography (hexane/EtOAc $20: 1$ to $5: 1$ ) yielded 6 (405 $\mathrm{mg}, 96 \%$ yield) as a colorless oil; $[\alpha]_{\mathrm{D}}{ }^{23}+83.7$ (c 1.06, $\mathrm{CHCl}_{3}$ ); IR (film) 3030, 2959, 2916, 2844, 2244, 1472, 1426, 1298, $1155 \mathrm{~cm}^{-1} ;{ }^{1} \mathrm{H} \mathrm{NMR}\left(300 \mathrm{MHz}, \mathrm{CDCl}_{3}\right) \delta 5.70-5.58(\mathrm{~m}, 2 \mathrm{H}), 3.66(\mathrm{dd}, J=11.4,4.9 \mathrm{~Hz}$, $1 \mathrm{H}), 3.56(\mathrm{dd}, J=11.4,4.1 \mathrm{~Hz}, 1 \mathrm{H}), 2.47\left(\mathrm{ABX}, \Delta v_{\mathrm{AB}}=10.6, J_{\mathrm{AB}}=16.9, J_{\mathrm{AX}}=7.2, J_{\mathrm{BX}}=4.2 \mathrm{~Hz}\right.$, 2H), 2.30-1.99 (m, 6H); ${ }^{13} \mathrm{C} \mathrm{NMR}\left(75 \mathrm{MHz}, \mathrm{CDCl}_{3}\right) \delta 126.1,125.2,118.9,47.8,38.3,32.0,29.7$, 28.0, 21.6; MS (EI) $m / z$ (relative intensity) $169\left(\mathrm{M}^{+},{ }^{35} \mathrm{Cl}, 11 \%\right), 171\left(\mathrm{M}^{+},{ }^{37} \mathrm{Cl}, 3 \%\right)$; Anal. Calcd for $\mathrm{C}_{9} \mathrm{H}_{12} \mathrm{ClN}$ : C, 63.72; H, 7.13; N, 8.26. Found: C, 63.95; H, 7.22; N, 8.09.

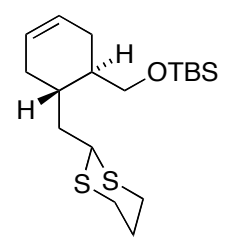

Dithiane (-)-S3. To a solution of 1,3-dithiane (409 $\mathrm{mg}, 3.41 \mathrm{mmol}$ ) in THF/HMPA $(10: 1 \mathrm{v} / \mathrm{v}, 8.8 \mathrm{~mL})$ was added $n$-BuLi $(1.6 \mathrm{M}$ in hexane, $2.20 \mathrm{~mL}$, $3.51 \mathrm{mmol})$ at $-78{ }^{\circ} \mathrm{C}$ under $\mathrm{N}_{2}$. After $1 \mathrm{~h}$, iodide (-)-3a $(220 \mathrm{mg}, 0.60 \mathrm{mmol})$ was added and the mixture was allowed to warm to room temperature over ca. $12 \mathrm{~h}$. The reaction mixture was partitioned between water and EtOAc, and the organic phase was dried $\left(\mathrm{Na}_{2} \mathrm{SO}_{4}\right)$ and concentrated. Flash chromatography (20:1 hexane/EtOAc) afforded dithiane (-)-S3 as a light brown oil $\left(210 \mathrm{mg}, 97 \%\right.$ yield). $[\alpha]_{\mathrm{D}}{ }^{28}-54.6$ (c 4.5, $\mathrm{CHCl}_{3}$ ); IR (film) 3022, 2952, 2927, 2855, 2897, 1597, 1471, 1422, 1251, $1105 \mathrm{~cm}^{-1} ;{ }^{1} \mathrm{H}$ NMR $\left(500 \mathrm{MHz}, \mathrm{CDCl}_{3}\right) \delta 5.58(\mathrm{ddd}, J=13.6,13.6,13.6 \mathrm{~Hz}, 2 \mathrm{H}), 4.10(\mathrm{dd}, J=9.0,5.7 \mathrm{~Hz}$, $1 \mathrm{H}), 3.56(\mathrm{~d}, J=6.0 \mathrm{~Hz}, 2 \mathrm{H}), 2.94-2.72(\mathrm{~m}, 4 \mathrm{H}), 2.26-2.15(\mathrm{~m}, 1 \mathrm{H}), 2.15-1.79(\mathrm{~m}, 6 \mathrm{H}), 1.77-$ $1.67(\mathrm{~m}, 1 \mathrm{H}), 1.67-1.53(\mathrm{~m}, 2 \mathrm{H}), 0.89(\mathrm{~s}, 9 \mathrm{H}), 0.04(\mathrm{~d}, J=3.8 \mathrm{~Hz}, 6 \mathrm{H}) ;{ }^{13} \mathrm{C} \mathrm{NMR}(125 \mathrm{MHz}$, $\left.\mathrm{CDCl}_{3}\right) \delta 125.8,124.9,64.9,45.3,39.5,39.0,30.6,30.5,30.2,28.8,26.3,26.1,25.9,18.2$, -5.4, 5.5; MS (CI) $\mathrm{m} / z$ (relative intensity) $359\left([\mathrm{M}+\mathrm{H}]^{+}, 100 \%\right)$; Anal. Calcd for $\mathrm{C}_{18} \mathrm{H}_{34} \mathrm{OS}_{2} \mathrm{Si}$ : C, 60.27; H, 9.55. Found: C, 60.53; H, 9.56. The antipode (+)-S3 was prepared by silylation of (+)S4 (TBSCl, imidazole, $\left.\mathrm{CH}_{2} \mathrm{Cl}_{2}\right)$ in $94 \%$ yield; $[\alpha]_{\mathrm{D}}^{25}+54.8\left(c 0.95, \mathrm{CHCl}_{3}\right)$. 


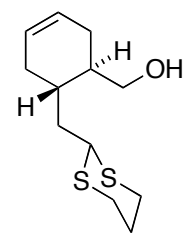

Alcohol (-)-S4. To a solution of dithiane (-)-S3 (30 mg, $0.083 \mathrm{mmol})$ in THF $(1.5 \mathrm{~mL})$ was added tetrabutylammonium fluoride $(1.0 \mathrm{M}$ in THF, $0.175 \mathrm{~mL}, 0.175$ $\mathrm{mmol}$ ). After $3 \mathrm{~h}$, concentration and gradient flash chromatography (hexane/EtOAc 1:1) afforded alcohol (-)-S4 (17 mg, 83\% yield) as a colorless oil; $[\alpha]_{\mathrm{D}}^{28}-59.8(c$ 2.2, $\mathrm{CHCl}_{3}$ ); IR (film) 3400, 3020, 2897, 1432, 1422, 1275, 1187, 1052, 1033, 908 $\mathrm{cm}^{-1} ;{ }^{1} \mathrm{H}$ NMR $\left(500 \mathrm{MHz}, \mathrm{CDCl}_{3}\right) \delta 5.68-5.51(\mathrm{~m}, 2 \mathrm{H}), 4.10(\mathrm{dd}, J=9.0,5.7 \mathrm{~Hz}, 1 \mathrm{H}), 3.68-3.56$ (m, 2H), 2.98-2.72 (m, 4H), 2.31-1.59 (m, 10H), $1.54(\mathrm{~s}, 1 \mathrm{H}) ;{ }^{13} \mathrm{C} \mathrm{NMR}\left(125 \mathrm{MHz}, \mathrm{CDCl}_{3}\right) \delta$ $125.0,125.4,65.0,45.3,39.1,38.9,30.5,30.4,30.3,28.5,26.0,25.8$; MS (CI) $\mathrm{m} / \mathrm{z}$ (relative intensity) $245\left([\mathrm{M}+\mathrm{H}]^{+}, 8 \%\right), 137(100 \%)$. The antipode $(+)-\mathbf{S} 4$ was prepared by the same procedure in $32 \%$ yield over two steps from $(+)-3 \mathbf{3} ;[\alpha]_{D}^{24}+53.3\left(c 1.13, \mathrm{CHCl}_{3}\right)$.

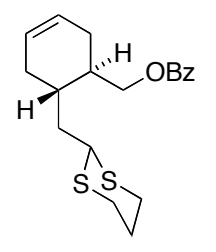

Benzoate (-)-S5. A solution of alcohol (-)-S4 (45mg, $0.183 \mathrm{mmol})$, benzoic anhydride ( $83 \mathrm{mg}, 0.366 \mathrm{mmol}$ ), 4-dimethylaminopyridine (DMAP) (10 mg, 0.08 mmol) and $\mathrm{Et}_{3} \mathrm{~N}(76 \mu \mathrm{L}, 0.55 \mathrm{mmol})$ in $\mathrm{CH}_{2} \mathrm{Cl}_{2}(2.0 \mathrm{~mL})$ was stirred for $1 \mathrm{~d}$. Concentration and flash chromatography (3:1 hexane/EtOAc) afforded benzoate $(-)$-S5 (56 mg, 87\% yield) as a colorless oil; $[\alpha]_{\mathrm{D}}^{30}-83.9\left(c 2.0, \mathrm{CHCl}_{3}\right)$; IR (film) 3063, 2899, 2842, 1789, 1718, 1451, 1314, 1274, 1175, 1026, $908 \mathrm{~cm}^{-1}$; ${ }^{1} \mathrm{H}$ NMR $\left(500 \mathrm{MHz}, \mathrm{CDCl}_{3}\right) \delta 8.05(\mathrm{~d}, J=7.2 \mathrm{~Hz}, 2 \mathrm{H}), 7.54(\mathrm{dd}, J=7.2,7.2 \mathrm{~Hz}, 1 \mathrm{H}), 7.43(\mathrm{dd}, J=8.3$, $8.3 \mathrm{~Hz}, 2 \mathrm{H}), 5.70-5.58(\mathrm{~m}, 2 \mathrm{H}), 4.40-4.25(\mathrm{~m}, 2 \mathrm{H}), 4.12(\mathrm{dd}, J=9.4,5.7 \mathrm{~Hz}, 1 \mathrm{H}), 2.94-2.74(\mathrm{~m}$, $4 \mathrm{H}), 2.37-2.27(\mathrm{~m}, 1 \mathrm{H}), 2.26-1.96(\mathrm{~m}, 6 \mathrm{H}), 1.92-1.76(\mathrm{~m}, 2 \mathrm{H}), 1.76-1.65(\mathrm{~m}, 1 \mathrm{H}) ;{ }^{13} \mathrm{C}$ NMR $(125$ $\left.\mathrm{MHz}, \mathrm{CDCl}_{3}\right) \delta 166.6,132.8,130.4,129.6,128.3,125.1,125.1,66.8,45.1,38.8,36.4,30.9$, 30.4, 30.2, 28.6, 26.4, 26.0; MS (CI) $\mathrm{m} / z$ (relative intensity) $349\left([\mathrm{M}+\mathrm{H}]^{+}, 92 \%\right), 227(100 \%)$; Anal. Calcd for $\mathrm{C}_{19} \mathrm{H}_{24} \mathrm{O}_{2} \mathrm{~S}_{2}$ : C, 65.48; H, 6.94. Found: C, 65.46; H, 7.04. The antipode was prepared by the same procedure from alcohol (+)-S4 in 98\% yield; $[\alpha]_{\mathrm{D}}^{24}+80.9\left(c 1.11, \mathrm{CHCl}_{3}\right)$.

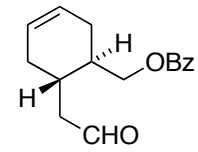

Aldehyde (-)-S6. A solution of dithiane (-)-S5 (44 mg, $0.130 \mathrm{mmol})$ and $\mathrm{Hg}\left(\mathrm{ClO}_{4}\right)_{2}\left(0.4 \mathrm{M}\right.$ in $\left.\mathrm{H}_{2} \mathrm{O}, 0.47 \mathrm{~mL}, 0.188 \mathrm{mmol}\right)$ in $\mathrm{THF}(3.0 \mathrm{~mL})$ was stirred for 3 h. Concentration and flash chromatography (1:1 hexane/EtOAc) afforded aldehyde (-)-S6 (30 mg, 89\% yield) as a colorless oil; $[\alpha]_{\mathrm{D}}{ }^{31}-41.9\left(c 1.6, \mathrm{CHCl}_{3}\right)$; IR (film) 3027, 2901, 2839, 2722, 1720, 1451, 1389, 1314, 1273, 1115, $1070 \mathrm{~cm}^{-1} ;{ }^{1} \mathrm{H}$ NMR (500 MHz, $\left.\mathrm{CDCl}_{3}\right) \delta 9.81(\mathrm{dd}, J=1.5,1.5 \mathrm{~Hz}, 1 \mathrm{H}), 8.03(\mathrm{~d}, J=8.3 \mathrm{~Hz}, 2 \mathrm{H}), 7.56(\mathrm{~m}, J=7.2,1.1 \mathrm{~Hz}, 1 \mathrm{H})$, $7.44(\mathrm{dd}, J=7.9,7.9 \mathrm{~Hz}, 2 \mathrm{H}), 5.72-5.60(\mathrm{~m}, 2 \mathrm{H}), 4.37-4.26(\mathrm{~m}, 2 \mathrm{H}), 2.64$ (ddd, $J=16.2,4.9,1.5$ $\mathrm{Hz}, 1 \mathrm{H}), 2.46$ (ddd, $J=16.6,8.3,2.6 \mathrm{~Hz}, 1 \mathrm{H}), 2.43-2.35(\mathrm{~m}, 1 \mathrm{H}), 2.33-2.17(\mathrm{~m}, 2 \mathrm{H}), 2.12-1.99$ (m, 2H) $1.93-1.81(\mathrm{~m}, 1 \mathrm{H}) ;{ }^{13} \mathrm{C}$ NMR $\left(125 \mathrm{MHz}, \mathrm{CDCl}_{3}\right) \delta$ 166.5, 133.0, 130.2, 129.5, 128.4, 125.2, 125.1, 66.7, 47.6, 36.4, 29.3, 29.2, 26.6; MS (CI) $\mathrm{m} / \mathrm{z}$ (relative intensity) 258 (M, 2\%), $256(75 \%), 137(100 \%) . \quad$ The antipode (+)-S6 was prepared from benzoate (+)-S5 (MeI, acetonitrile/water) in $45 \%$ yield; $[\alpha]_{\mathrm{D}}{ }^{25}+44.5\left(c 1.33, \mathrm{CHCl}_{3}\right)$.

Preparation of Hydrazones (General Procedure A). To a solution of 3-amino-4phenylmethyl-2-oxazolidone in $\mathrm{CH}_{2} \mathrm{Cl}_{2}$ was added $\mathrm{TsOH} \bullet \mathrm{H}_{2} \mathrm{O}(5 \mathrm{~mol} \%)$ and the appropriate aldehyde at room temperature. When the reaction was complete (TLC), concentration and flash chromatography (e.g., hexane/EtOAc 4:1 to 1:1) furnished the hydrazone. Only the (E)-isomer was detected. 


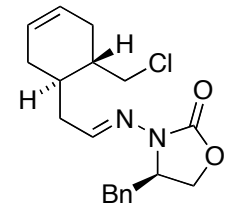

$(R)$-4-benzyl-3-((E)-2-((1R,6S)-6-(chloromethyl)cyclohex-3-enyl)ethylidene-amino) oxazolidin-2-one (8a). To a solution of 6 (339 $\mathrm{mg}, 1.99 \mathrm{mmol})$ in toluene $(16 \mathrm{~mL})$ was added DIBALH $(20 \% \mathrm{w} / \mathrm{w}$ solution in toluene, $1.63 \mathrm{~g}, 2.3$ $\mathrm{mmol})$ at $-78{ }^{\circ} \mathrm{C}$. After $30 \mathrm{~min}$, the reaction was quenched with saturated aqueous sodium potassium tartrate, and the mixture was filtered through celite. The filtrate was washed with brine, dried $\left(\mathrm{Na}_{2} \mathrm{SO}_{4}\right)$, concentrated and purified by gradient flash chromatography (hexane to hexane/EtOAc 20:1) to afford the corresponding aldehyde (253 $\mathrm{mg}$, $74 \%$ yield $)$ as a colorless oil; $[\alpha]_{\mathrm{D}}{ }^{23}+41.2\left(c 0.58, \mathrm{CHCl}_{3}\right)$; IR (film) 3027, 2922, 2847, 1723, $1425,1295 \mathrm{~cm}^{-1}$; ${ }^{1} \mathrm{H}$ NMR $\left(300 \mathrm{MHz}, \mathrm{CDCl}_{3}\right) \delta 9.79(\mathrm{t}, J=1.83,1 \mathrm{H}), 5.70-5.51(\mathrm{~m}, 2 \mathrm{H}), 3.56$ $\left(\mathrm{ABX}, \Delta v_{\mathrm{AB}}=8.2, J_{\mathrm{AB}}=11.1, J_{\mathrm{AX}}=6.1, J_{\mathrm{BX}}=5.1 \mathrm{~Hz}, 2 \mathrm{H}\right), 2.59-2.52(\mathrm{~m}, 1 \mathrm{H}), 2.42-2.37(\mathrm{~m}$, $2 \mathrm{H}), 2.25-2.10(\mathrm{~m}, 3 \mathrm{H}), 1.94-1.88(\mathrm{~m}, 2 \mathrm{H}) ;{ }^{13} \mathrm{C} \mathrm{NMR}\left(75 \mathrm{MHz}, \mathrm{CDCl}_{3}\right)$ \& 202.1, 125.2, 125.0, 47.8, 47.4, 38.8, 29.3, 28.9, 27.2; MS (EI) $\mathrm{m} / z$ (relative intensity) $172\left(\mathrm{M}^{+},<1 \%\right), 128$ ([M-44] $]^{+}$, $30 \%){ }^{4}$ From this aldehyde $(245 \mathrm{mg}, 1.41 \mathrm{mmol})$ and $(R)$-3-amino-4-phenylmethyl-2oxazolidone (272 mg, $1.41 \mathrm{mmol}$ ) by General Procedure A was obtained 8a $(462 \mathrm{mg}, 95 \%)$ as colorless liquid; $[\alpha]_{\mathrm{D}}{ }^{24}+47.8$ (c $1.45 \mathrm{CHCl}_{3}$ ); IR (film) 3012, 2905, 1775, 1764, 1755, 1432, $1402,1207 \mathrm{~cm}^{-1} ;{ }^{1} \mathrm{H}$ NMR $\left(300 \mathrm{MHz}, \mathrm{CDCl}_{3}\right) \delta 8.09(\mathrm{t}, J=5.8 \mathrm{~Hz}, 1 \mathrm{H}), 7.38-7.29(\mathrm{~m}, 3 \mathrm{H})$, 7.21-7.18(m, 2H), 5.70-5.60 (m, 2H), 4.45-4.38 (m, 1H), $4.26(\mathrm{dd}, J=8.7,8.1 \mathrm{~Hz}, 1 \mathrm{H}), 4.11(\mathrm{dd}$, $J=8.8,5.8 \mathrm{~Hz}, 1 \mathrm{H}), 3.68\left(\mathrm{ABX}, \Delta v_{\mathrm{AB}}=25.1, J_{\mathrm{AB}}=11.0, J_{\mathrm{AX}}=6.1, J_{\mathrm{BX}}=4.6 \mathrm{~Hz}, 2 \mathrm{H}\right), 3.23(\mathrm{dd}$, $J=13.8,3.7 \mathrm{~Hz}, 1 \mathrm{H}), 2.85(\mathrm{dd}, J=13.8,8.6 \mathrm{~Hz}, 1 \mathrm{H}), 2.60(\mathrm{ddd}, J=14.4,5.3,5.3, \mathrm{~Hz} 1 \mathrm{H}), 2.42$ (ddd, $J=14.3,7.6,6.4 \mathrm{~Hz}, 1 \mathrm{H}), 2.26-2.15(\mathrm{~m}, 4 \mathrm{H}), 1.99-1.93(\mathrm{~m}, 2 \mathrm{H}) ;{ }^{13} \mathrm{C}$ NMR $(75 \mathrm{MHz}$, $\mathrm{CDCl}_{3}$ ) $\delta 155.5,154.6,135.4,129.2,129.3,127.4,125.30,125.26,65.9,57.9,48.2,39.0,37.5$, 36.8, 32.4, 29.0, 27.4; MS (ESI) $\mathrm{m} / \mathrm{z}$ (relative intensity) $347.09\left([\mathrm{M}+\mathrm{H}]^{+}, 100 \%\right), 369.08$ $\left([\mathrm{M}+\mathrm{Na}]^{+}, 52 \%\right)$; Anal. Calcd for $\mathrm{C}_{19} \mathrm{H}_{23} \mathrm{ClN}_{2} \mathrm{O}_{2}: \mathrm{C}, 65.79 ; \mathrm{H}, 6.68 ; \mathrm{N}, 8.08$. Found: C, 65.90; H, $6.65 ; \mathrm{N}, 8.09$.

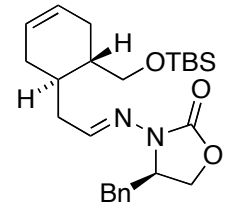

(R)-4-benzyl-3-((E)-2-((1R,6S)-6-((tert-butyldimethylsilyloxy)methyl)cyclohex-3-enyl)ethylideneamino)oxazolidin-2-one (8b). To a solution of 4 $(155 \mathrm{mg}, 0.58 \mathrm{mmol})$ in toluene $(2.5 \mathrm{~mL})$ was added DIBALH $(1 \mathrm{M}$ in hexane, $0.8 \mathrm{~mL}, 0.8 \mathrm{mmol})$ at $-78{ }^{\circ} \mathrm{C}$. After $30 \mathrm{~min}$, the reaction was quenched with saturated aqueous sodium potassium tartrate, and the mixture was filtered through celite. The filtrate was washed with brine, dried $\left(\mathrm{Na}_{2} \mathrm{SO}_{4}\right)$, concentrated and purified by gradient flash chromatography (hexane to 20:1 hexane/EtOAc) to afford the corresponding aldehyde (120 $\mathrm{mg}, 77 \%)$ as a colorless oil. From this aldehyde $(75 \mathrm{mg}, 0.27 \mathrm{mmol})$ and $(R)$-3-amino-4phenylmethyl-2-oxazolidinone $(53 \mathrm{mg}, 0.27 \mathrm{mmol}$ ) by General Procedure A was obtained $\mathbf{8 b}$ (100 mg, 81\% yield) as a colorless liquid; $[\alpha]_{\mathrm{D}}^{23}+41.7$ (c 2.46, $\mathrm{CHCl}_{3}$ ); IR (film) 3025, 2953, 2899, 2855, 1776, 1755, 1471, 1399, 1250, 1207, $1099 \mathrm{~cm}^{-1} ;{ }^{1} \mathrm{H}$ NMR $\left(300 \mathrm{MHz}, \mathrm{CDCl}_{3}\right) \delta 8.03$ $(\mathrm{dd}, J=6.2,5.6 \mathrm{~Hz}, 1 \mathrm{H}), 7.30-7.18(\mathrm{~m}, 3 \mathrm{H}), 7.12-7.10(\mathrm{~m}, 2 \mathrm{H}), 5.62-5.52(\mathrm{~m}, 2 \mathrm{H}), 4.31-4.28$ $(\mathrm{m}, 1 \mathrm{H}), 4.17(\mathrm{dd}, J=8.7,7.9 \mathrm{~Hz}, 1 \mathrm{H}), 4.02(\mathrm{dd}, J=8.7,5.7 \mathrm{~Hz}, 1 \mathrm{H}), 3.58\left(\mathrm{ABX}, \Delta v_{\mathrm{AB}}=7.1\right.$, $\left.J_{\mathrm{AB}}=10.1, J_{\mathrm{AX}}=6.2, J_{\mathrm{BX}}=5.0 \mathrm{~Hz}, 2 \mathrm{H}\right), 3.17(\mathrm{dd}, J=13.7,3.5 \mathrm{~Hz}, 1 \mathrm{H}), 2.74(\mathrm{dd}, J=13.7,8.8$ $\mathrm{Hz}, 1 \mathrm{H}), 2.55$ (ddd, $J=14.3,5.3,5.3 \mathrm{~Hz}, 1 \mathrm{H}), 2.32(\mathrm{ddd}, J=14.7,8.5,6.4 \mathrm{~Hz}, 1 \mathrm{H}), 2.20-1.75$ $(\mathrm{m}, 5 \mathrm{H}), 1.70-1.60(\mathrm{~m}, 1 \mathrm{H}), 0.83(\mathrm{~s}, 9 \mathrm{H}), 0.00(\mathrm{~s}, 6 \mathrm{H}) ;{ }^{13} \mathrm{C} \mathrm{NMR}\left(75 \mathrm{MHz}, \mathrm{CDCl}_{3}\right) \delta 157.0$, 154.7, 135.5, 129.4, 129.1, 127.4, 126.1, 125.3, 65.9, 64.9, 58.1, 39.5, 37.5, 37.2, 32.2, 29.4, 26.8, 26.1, 18.5, -5.20, -5.24; MS (ESI) $m / z$ (relative intensity) $442.97\left([\mathrm{M}+\mathrm{H}]^{+} 44 \%\right)$; Anal. Calcd for $\mathrm{C}_{25} \mathrm{H}_{38} \mathrm{~N}_{2} \mathrm{O}_{3} \mathrm{Si}$ : C, 67.83; H, 8.65; N, 6.33. Found: C, 67.80; H, 8.76; N, 6.20. 


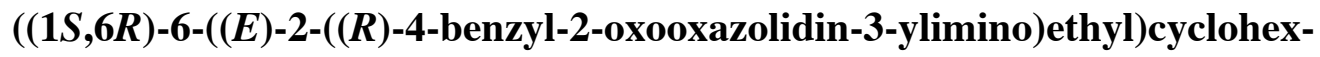
3-enyl)methyl benzoate (8c). From $(R)$-3-amino-4-phenylmethyl-2-oxazolidone (64 mg, $0.33 \mathrm{mmol})$, and aldehyde (+)-S6 $(86 \mathrm{mg}, 0.33 \mathrm{mmol})$ by General Procedure A was obtained $\mathbf{8 c}\left(137 \mathrm{mg}, 94 \%\right.$ yield) as a colorless liquid; $[\alpha]_{\mathrm{D}}{ }^{23}$ +45.6 (c 1.97, $\mathrm{CHCl}_{3}$ ); IR (film) 2904, 1776, 1755, 1720, 1402, 1273, 1209, 1113, $1099 \mathrm{~cm}^{-1} ;{ }^{1} \mathrm{H}$ NMR $\left(300 \mathrm{MHz}, \mathrm{CDCl}_{3}\right) \delta 8.1(\mathrm{t}, J=5.8,1 \mathrm{H}), 8.03-8.00(\mathrm{~m}, 2 \mathrm{H}), 7.55-7.50(\mathrm{~m}$, $1 \mathrm{H}), 7.43-7.38(\mathrm{~m}, 2 \mathrm{H}), 7.30-7.22(\mathrm{~m}, 3 \mathrm{H}), 7.13-7.11(\mathrm{~m}, 2 \mathrm{H}), 5.70-5.60(\mathrm{~m}, 2 \mathrm{H}), 4.41-4.26(\mathrm{~m}$, $3 \mathrm{H}), 4.18(\mathrm{dd}, J=8.7,8.7 \mathrm{~Hz}, 1 \mathrm{H}), 4.02(\mathrm{dd}, J=8.74,5.8 \mathrm{~Hz}, 1 \mathrm{H}), 3.18(\mathrm{dd}, J=13.7,3.6,1 \mathrm{H})$, $2.75(\mathrm{dd}, J=13.7,8.8 \mathrm{~Hz}, 1 \mathrm{H}), 2.61$ (ddd $J=14.5,5.3,5.3 \mathrm{~Hz}, 1 \mathrm{H}), 2.41$ (ddd, $J=14.4,7.8,6.3$ $\mathrm{Hz}, 1 \mathrm{H}), 2.28-1.87(\mathrm{~m}, 6 \mathrm{H}) ;{ }^{13} \mathrm{C}$ NMR $\left(75 \mathrm{MHz}, \mathrm{CDCl}_{3}\right) \delta 166.8,156.1,154.7,135.4,133.2$, 130.4, 129.7, 129.4, 129.1, 128.6, 127.4, 125.5, 125.4, 67.0, 65.9, 58.2, 37.6, 37.1, 36.6, 32.6, 29.3, 26.9; MS (ESI) $\mathrm{m} / z$ (relative intensity) $433.05\left([\mathrm{M}+1]^{+} 55 \%\right), 455.2\left([\mathrm{M}+\mathrm{Na}]^{+}, 100 \%\right)$; Anal. Calcd for $\mathrm{C}_{26} \mathrm{H}_{28} \mathrm{~N}_{2} \mathrm{O}_{4} ; \mathrm{C}, 72.20 ; \mathrm{H}, 6.53 ; \mathrm{N}, 6.48$. Found: C, 72.14; H, 6.65; N, 6.20.

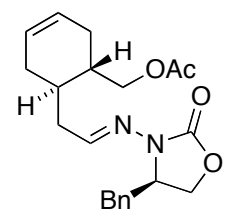

((1S,6R)-6-((E)-2-((R)-4-benzyl-2-oxooxazolidin-3-ylimino)ethyl)cyclohex3-enyl)methyl acetate (8d). A solution of alcohol (+)-S4 (160 mg. $0.65 \mathrm{mmol})$, acetic anhydride $(75 \mu \mathrm{L}, 0.78 \mathrm{mmol})$, triethylamine $(0.13 \mathrm{~mL}, 0.97 \mathrm{mmol})$, and DMAP (20 mg, $0.1 \mathrm{mmol})$ in $\mathrm{CH}_{2} \mathrm{Cl}_{2}(3 \mathrm{~mL})$ was stirred at room temperature for $1 \mathrm{~h}$, then partitioned between $\mathrm{CH}_{2} \mathrm{Cl}_{2}(10 \mathrm{~mL})$ and water. The organic phase was washed with brine, dried $\left(\mathrm{Na}_{2} \mathrm{SO}_{4}\right)$, and concentrated. Flash chromatography (hexane to hexane EtOAc 9:1) afforded the acetate derivative $(168 \mathrm{mg}, 92 \%)$ as a colorless oil, which was immediately subjected to dithiane hydrolysis. To a solution of this dithiane in acetonitrile/water (4:1, $5 \mathrm{~mL})$ was added methyl iodide $(75 \mu \mathrm{L}, 1.2 \mathrm{mmol})$. After $1 \mathrm{~d}$, concentration and flash chromatography (hexane to hexane EtOAc 20:1) afforded the corresponding aldehyde (62 mg, $53 \%)$ as a colorless oil. From this aldehyde and $(R)$-3-amino-4-phenylmethyl-2-oxazolidinone (62 mg, $0.32 \mathrm{mmol}$ ) by General Procedure A was obtained 8d (113 mg, 95\% yield) as colorless liquid. The overall yield was $46 \%$ for 3 steps from (+)-S4. $[\alpha]_{\mathrm{D}}{ }^{25}+47.4\left(c, 1.45 \mathrm{CHCl}_{3}\right)$; IR (film) 3026, 2903, 1776, 1754, 1742, 1730, 1401, 1241, $1031 \mathrm{~cm}^{-1} ;{ }^{1} \mathrm{H}$ NMR $\left(300 \mathrm{MHz}, \mathrm{CDCl}_{3}\right)$ $\delta 8.20(\mathrm{t}, J=5.8 \mathrm{~Hz}, 1 \mathrm{H}), 7.43-7.34(\mathrm{~m}, 3 \mathrm{H}), 7.26-7.23(\mathrm{~m}, 2 \mathrm{H}), 5.75-5.68(\mathrm{~m}, 2 \mathrm{H}), 4.48-4.40$ $(\mathrm{m}, 1 \mathrm{H}), 4.31(\mathrm{dd}, J=8.7,8.0 \mathrm{~Hz}, 1 \mathrm{H}), 4.25-4.11(\mathrm{~m}, 3 \mathrm{H}), 3.31(\mathrm{dd}, J=13.7,3.6 \mathrm{~Hz}, 1 \mathrm{H}), 2.89$ (dd, $J=13.7,8.7 \mathrm{~Hz}, 1 \mathrm{H}$ ), 2.64 (ddd, $J=14.5,5.4,5.4 \mathrm{~Hz}, 1 \mathrm{H}$ ), 2.47 (ddd, $J=14.4,8.0,6.2 \mathrm{~Hz}$, $1 \mathrm{H}), 2.30-2.25(\mathrm{~m}, 2 \mathrm{H}), 2.15(\mathrm{~s}, 3 \mathrm{H}), 2.14-1.96(\mathrm{~m}, 4 \mathrm{H}) ;{ }^{13} \mathrm{C} \mathrm{NMR}\left(75 \mathrm{MHz}, \mathrm{CDCl}_{3}\right) \delta 171.3$, 156.1, 154.6, 135.4, 129.4, 129.0, 127.3, 125.3, 125.2, 66.5, 65.9, 58.2, 37.6, 37.0, 36.2, 32.3, 28.9, 26.5, 21.1; MS (ESI) $\mathrm{m} / z$ (relative intensity) $371.07\left([\mathrm{M}+1]^{+}, 68 \%\right), 393.17\left([\mathrm{M}+\mathrm{Na}]^{+}\right.$, $100 \%)$.

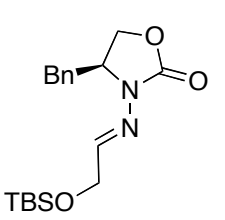

(S,E)-4-benzyl-3-(2-(tert-butyldimethylsilyloxy)ethylideneamino)oxazolidin-2-one (16). From $(S)$-3-amino-4-phenylmethyl-2-oxazolidinone $(760 \mathrm{mg}$, $3.95 \mathrm{mmol}$ ), and glycolaldehyde dimer (475 $\mathrm{mg}, 3.95 \mathrm{mmol})$ by General Procedure A was obtained the glycolaldehyde hydrazone $(825 \mathrm{mg}, 89 \%)$ as a colorless liquid. To this alcohol $(825 \mathrm{mg}, 3.52 \mathrm{mmol})$ and imidazole $(480 \mathrm{mg}$, $7.04 \mathrm{mmol})$ in a solution of $\mathrm{CH}_{2} \mathrm{Cl}_{2}(16 \mathrm{~mL})$ was added tert-butyldimethylsilyl chloride $(530 \mathrm{mg}$, $3.52 \mathrm{mmol}$ ) at room temperature. After $4 \mathrm{~h}$, the reaction mixture was diluted with $\mathrm{CH}_{2} \mathrm{Cl}_{2}$, washed successively with water and brine, and dried $\left(\mathrm{Na}_{2} \mathrm{SO}_{4}\right)$. Concentration and flash chromatography (hexane/EtOAc 9:1 to 1:1) afforded $\mathbf{1 6}(1.12 \mathrm{~g}, 92 \%$ yield) as a colorless liquid; $[\alpha]_{\mathrm{D}}^{23}+8.76\left(c 1.78, \mathrm{CHCl}_{3}\right)$; IR (film) 3029, 2953, 2928, 2856, 1777, 1764, 1472, 1406, 1361, 
1251, $1099 \mathrm{~cm}^{-1} ;{ }^{1} \mathrm{H}$ NMR $\left(300 \mathrm{MHz}, \mathrm{CDCl}_{3}\right) \delta 7.90(\mathrm{t}, J=4.7 \mathrm{~Hz}, 1 \mathrm{H}), 7.23-7.14(\mathrm{~m}, 3 \mathrm{H})$, $7.05-7.03(\mathrm{~m}, 2 \mathrm{H}), 4.29(\mathrm{~d}, J=4.6 \mathrm{~Hz}, 2 \mathrm{H}), 4.26-4.23(\mathrm{~m}, 1 \mathrm{H}), 4.12(\mathrm{t}, J=8.8 \mathrm{~Hz}, 1 \mathrm{H}), 3.99(\mathrm{dd}$, $J=8.8,5.0 \mathrm{~Hz}, 1 \mathrm{H}), 3.12(\mathrm{dd}, J=13.8,3.4 \mathrm{~Hz}, 1 \mathrm{H}), 2.68(\mathrm{dd}, J=13.8,8.8 \mathrm{~Hz}, 1 \mathrm{H}), 0.80(\mathrm{~s}$, 9H), 0.00 (s, 6H); ${ }^{13} \mathrm{C} \mathrm{NMR}\left(75 \mathrm{MHz}, \mathrm{CDCl}_{3}\right) \delta$ 154.1, 152.7, 135.2, 129.4, 129.1, 127.5, 65.9, 63.9, 57.4, 37.0, 26.0, 18.5, -5.0 (2C); MS (ESI) $\mathrm{m} / z$ (relative intensity) $348\left(\mathrm{M}^{+}, 34 \%\right)$.

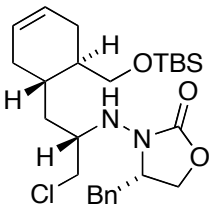

(S)-4-benzyl-3-((S)-3-chloro-1-((1R,6S)-6-(tert-butyldimethylsilyloxymethyl)cyclohex-3-enyl)propan-2-ylamino)oxazolidin-2-one (10). A mixture of hydrazone $9(75 \mathrm{mg}, 0.297 \mathrm{mmol})$ and $\mathrm{InCl}_{3}(106 \mathrm{mg}, 0.476 \mathrm{mmol})$ in benzene $/ \mathrm{CH}_{3} \mathrm{CN}(10: 1 \mathrm{v} / \mathrm{v}, 2.75 \mathrm{~mL})$ was stirred at room temperature under $\mathrm{N}_{2}$ for $40 \mathrm{~min}$. Then $\mathrm{Mn}_{2}(\mathrm{CO})_{10}(186 \mathrm{mg}, 0.476 \mathrm{mmol})$ and iodide (-)-3a (87 mg, 0.238 mmol) were added and the mixture was irradiated for $13 \mathrm{~h}$. The reaction mixture was partitioned between $\mathrm{CH}_{2} \mathrm{Cl}_{2}$ and aqueous $\mathrm{NH}_{4} \mathrm{OH}$. The organic phase was dried $\left(\mathrm{Na}_{2} \mathrm{SO}_{4}\right)$, concentrated and filtered through a short column of silica gel to remove most of the Mn residues. A mixture of the crude product and DBU $(50 \mu \mathrm{L})$ in EtOAc was stirred for $30 \mathrm{~min}$. Concentration and flash chromatography (3:1 hexane/EtOAc) afforded hydrazine $\mathbf{1 0}$ (30 mg, 26\% yield) as colorless oil; $[\alpha]_{\mathrm{D}}{ }^{31}-40.0\left(c 1.2, \mathrm{CHCl}_{3}\right)$; IR (film) 3301, 3026, 2954, 2928, 2856, 1760, 1471, 1401, 1361, 1252, $1099 \mathrm{~cm}^{-1} ;{ }^{1} \mathrm{H}$ NMR $\left(500 \mathrm{MHz}, \mathrm{CDCl}_{3}\right) \delta$ 7.35-7.15 (m, 5H), 5.68-5.61 (m, 2H), 4.22-4.09 $(\mathrm{m}, 2 \mathrm{H}), 4.05(\mathrm{dd}, J=8.7,4.5 \mathrm{~Hz}, 1 \mathrm{H}), 4.02-3.92(\mathrm{~m}, 1 \mathrm{H}), 3.74-3.52(\mathrm{~m}, 4 \mathrm{H}), 3.52-3.42$ (br s, $1 \mathrm{H}), 3.37(\mathrm{dd}, J=13.2,3.4 \mathrm{~Hz}, 1 \mathrm{H}), 2.58(\mathrm{dd}, J=13.6,9.8 \mathrm{~Hz}, 1 \mathrm{H}), 2.38(\mathrm{~m}$, apparent br d, $J=$ $18.1 \mathrm{~Hz}, 1 \mathrm{H}), 2.14-1.90(\mathrm{~m}, 3 \mathrm{H}), 1.85-1.71(\mathrm{~m}, 2 \mathrm{H}), 1.68-1.57(\mathrm{~m}, 1 \mathrm{H}), 1.56-1.44(\mathrm{~m}, 1 \mathrm{H}), 0.90$ (s, 9H), 0.05 (s, 3H), 0.04 (s, 3H); $\left.{ }^{13} \mathrm{C} \mathrm{NMR} \mathrm{(125} \mathrm{MHz,} \mathrm{CDCl}_{3}\right) \delta 158.6,135.8,129.1,128.8$, 127.0, 125.8, 125.1, 65.8, 64.6, 60.1, 57.9, 47.0, 39.9, 36.8, 34.4, 29.7, 29.1, 26.6, 25.9, 18.3, 5.5 (2C); MS (CI) $m / z$ (relative intensity) $494\left([\mathrm{M}+\mathrm{H}]^{+}, 100 \%\right)$.

Radical Addition (General Procedure B): Using standard pyrex glassware, a solution of the hydrazone in $\mathrm{CH}_{2} \mathrm{Cl}_{2}(0.02 \mathrm{M})$ was deoxygenated $\left(\mathrm{N}_{2}\right.$ or $\mathrm{Ar}$ was bubbled through the solution via a syringe needle for ca. $30 \mathrm{~min}$ ); then $\mathrm{InCl}_{3}$ was added (2.2 equiv). After $40 \mathrm{~min}$ at room temperature, the appropriate alkyl iodide (1.2-10 equiv) and $\mathrm{Mn}_{2}(\mathrm{CO})_{10}(1.2 \mathrm{eq})$ were added and the mixture was irradiated (300 $\mathrm{nm}$, Rayonet photochemical reactor) for 10-20 h. Triethylamine (5 equiv) was added, and the mixture was stirred for $40 \mathrm{~min}$ and concentrated. Flash chromatography (hexane/EtOAc $9: 1$ to $1: 1$ ) afforded $N$-acylhydrazines. The minor diastereomers were not detected $(<5 \%)$.

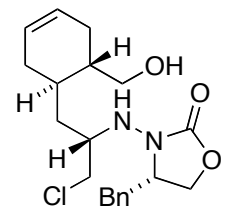

(S)-4-benzyl-3-((S)-3-chloro-1-((1R,6S)-6-(hydroxymethyl)cyclohex-3enyl)propan-2-ylamino)oxazolidin-2-one (11). From $9^{5}$ (47 mg, $0.18 \mathrm{mmol}$ ) and iodide $\mathbf{3 b}(85 \mathrm{mg}, 0.23 \mathrm{mmol})$ by General Procedure B was obtained 11 (21 $\mathrm{mg}, 30 \%$ yield) as a colorless oil; $[\alpha]_{\mathrm{D}}{ }^{25}+26.2\left(c 1.11, \mathrm{CHCl}_{3}\right)$; IR (film) 3436, $3289,3019,2910,1754,1744,1437,1402,1233,1097,1029 \mathrm{~cm}^{-1} ;{ }^{1} \mathrm{H}$ NMR $(400$ $\left.\mathrm{MHz}, \mathrm{DMSO}, 70{ }^{\circ} \mathrm{C}\right) \delta$ 7.32-7.29 (m, 2H), 7.24-7.21 (m, 3H), 5.63-5.58 (m, 2H), 4.24-4.17 (m, $1 \mathrm{H}), 4.06-3.98(\mathrm{~m}, 2 \mathrm{H}), 3.75-3.65(\mathrm{~m}, 2 \mathrm{H}), 3.47$ (dd, $J=10.4,5.6 \mathrm{~Hz}, 1 \mathrm{H}), 3.40-3.33(\mathrm{~m}, 2 \mathrm{H})$, $3.18(\mathrm{dd}, J=13.5,3.1 \mathrm{~Hz}, 1 \mathrm{H}), 2.72(\mathrm{dd}, J=13.6,8.7 \mathrm{~Hz}, 1 \mathrm{H}), 2.18(\mathrm{~m}$, apparent br d, $J=18$ $\mathrm{Hz}, 1 \mathrm{H}), 2.09$ (m, apparent br d, $J=17.4 \mathrm{~Hz}, 1 \mathrm{H}), 1.92-1.53(\mathrm{~m}, 5 \mathrm{H}), 1.40-1.33(\mathrm{~m}, 1 \mathrm{H}) ;{ }^{13} \mathrm{C}$ NMR $\left(100 \mathrm{MHz}, \mathrm{DMSO}, 70{ }^{\circ} \mathrm{C}\right) \delta 157.2,136.1,128.8,128.1,126.1,125.3,124.7,65.1,63.0$, 58.3, 57.1, 46.6, 38.8, 36.1, 33.8, 29.7, 28.5, 25.3; MS (ESI) $\mathrm{m} / z$ (relative intensity) 379.08 
$\left([\mathrm{M}+\mathrm{H}]^{+} 66 \%\right.$ ); HRMS (ESI) $\mathrm{m} / z$ calcd. for $\mathrm{C}_{20} \mathrm{H}_{27} \mathrm{~N}_{2} \mathrm{O}_{3} \mathrm{NaCl} 401.1608\left([\mathrm{M}+\mathrm{Na}]^{+}\right)$; found 401.1600 .

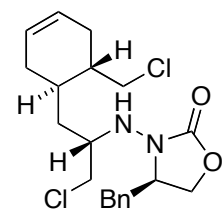

(R)-4-benzyl-3-((S)-3-chloro-1-((1R,6S)-6-(chloromethyl)cyclohex-3enyl)propan-2-ylamino)oxazolidin-2-one (12a). From hydrazone 8a (46 mg, $0.13 \mathrm{mmol})$ and chloroiodomethane $(0.09 \mathrm{~mL}, 1.3 \mathrm{mmol})$ by General Procedure B was obtained 12a (26 mg, 49\% yield) as a colorless oil; $[\alpha]_{\mathrm{D}}^{23}+6.0\left(c 0.9 \mathrm{CHCl}_{3}\right)$; IR (film) 3286, 3026, 2916, 1754, 1432, 1401, 1218, 1092, $1027 \mathrm{~cm}^{-1} ;{ }^{1} \mathrm{H}$ NMR $\left(300 \mathrm{MHz}, \mathrm{CDCl}_{3}\right) \delta$ 7.35-7.23 (m, 3H), 7.18-7.16 (m, 2H), 5.67-5.59 (m, 2H), $4.15(\mathrm{dd}, J=8.8$, $7.5 \mathrm{~Hz}, 2 \mathrm{H}), 4.05(\mathrm{dd}, J=8.9,5.2 \mathrm{~Hz}, 1 \mathrm{H}), 3.96-3.88(\mathrm{~m}, 1 \mathrm{H}), 3.70-3.57(\mathrm{~m}, 4 \mathrm{H}), 3.44(\mathrm{dd}, J=$ $13.5,3.6 \mathrm{~Hz}, 1 \mathrm{H}), 3.41-3.34(\mathrm{~m}, 1 \mathrm{H}), 2.61(\mathrm{dd}, J=13.3,10.1 \mathrm{~Hz}, 1 \mathrm{H}), 2.26-2.08(\mathrm{~m}, 3 \mathrm{H}), 2.05-$ $1.76(\mathrm{~m}, 4 \mathrm{H}) 1.39(\mathrm{ddd}, J=14.1,7.7,6.6 \mathrm{~Hz}, 1 \mathrm{H}) ;{ }^{13} \mathrm{C} \mathrm{NMR}\left(75 \mathrm{MHz}, \mathrm{CDCl}_{3}\right) \delta 159.4,135.9$, 129.3, 129.2, 127.3, 125.23, 125.15, 66.4, 60.0, 58.5, 48.3, 46.2, 39.0, 37.2, 34.4, 30.7, 29.1, 27.0; MS (ESI) $\mathrm{m} / \mathrm{z}$ (relative intensity) $397.05\left([\mathrm{M}+\mathrm{H}]^{+} 100 \%\right)$ HRMS (ESI) m/z: [M+Na] ${ }^{+}$ calcd. For $\mathrm{C}_{20} \mathrm{H}_{26} \mathrm{~N}_{2} \mathrm{O}_{2} \mathrm{NaCl}_{2}$ 419.1269; found 419.1294.

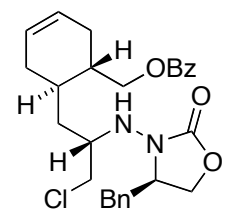

((1S,6R)-6-((S)-2-((R)-4-benzyl-2-oxazolidin-3-ylamino)-3-chloropropyl)cyclohex-3-enyl)methyl benzoate (12c). From 8c $(80 \mathrm{mg}, 0.18 \mathrm{mmol})$ and chloroiodomethane $(0.13 \mathrm{~mL}, 1.8 \mathrm{mmol})$ by General Procedure B was obtained 12c (32 mg, 36\% yield) as a colorless oil; $[\alpha]_{\mathrm{D}}^{23}+9.8\left(c 0.23 \mathrm{CHCl}_{3}\right)$; IR (film) 3276, 2917, 2851, 1752, 1724, 1711, 1270, $1096 \mathrm{~cm}^{-1} ;{ }^{1} \mathrm{H}$ NMR $(300 \mathrm{MHz}$, $\left.\mathrm{CDCl}_{3}\right) \delta 8.08-8.02(\mathrm{~m}, 2 \mathrm{H})$ 7.57-7.51 (m, 1H), 7.44-7.38 (m, 2H), 7.32-7.15 (m, 3H), 7.15-7.13 $(\mathrm{m}, 2 \mathrm{H}), 5.72-5.63(\mathrm{~m}, 2 \mathrm{H}), 4.38-4.35(\mathrm{~m}, 2 \mathrm{H}), 4.16-4.09(\mathrm{~m}, 2 \mathrm{H}), 4.02(\mathrm{dd}, J=8.8,5.6 \mathrm{~Hz}, 1 \mathrm{H}$ ), 3.93-3.85 (m, 1H), 3.64 (ABX, $\left.\Delta v_{\mathrm{AB}}=18.9, J_{\mathrm{AB}}=11.5, J_{\mathrm{AX}}=4.4, J_{\mathrm{BX}}=4.4 \mathrm{~Hz}, 2 \mathrm{H}\right), 3.47-3.40$ (m, 1H), 3.39 (dd, $J=13.2,3.6 \mathrm{~Hz}, 1 \mathrm{H}), 2.60$ (dd, $J=13.3,10.0, \mathrm{~Hz}, 1 \mathrm{H}), 2.34-2.21(\mathrm{~m}, 2 \mathrm{H})$, 2.09-1.80 (m, 5H), 1.50-1.40 (m, 1H); ${ }^{13} \mathrm{C} \mathrm{NMR}\left(75 \mathrm{MHz}, \mathrm{CDCl}_{3}\right) \delta 166.9,159.5,135.8,133.2$, 129.8, 129.3, 129.22, 129.16, 128.6, 127.4, 125.5, 125.3, 67.0, 66.5, 60.0, 58.0, 46.0, 37.3, 36.7, 34.3, 31.0, 29.5, 26.6; MS (ESI) $\mathrm{m} / z$ (relative intensity) $483.09\left([\mathrm{M}+\mathrm{H}]^{+} 57 \%\right.$ ); HRMS (ESI) $\mathrm{m} / z$ calcd. for $\mathrm{C}_{27} \mathrm{H}_{31} \mathrm{~N}_{2} \mathrm{O}_{4} \mathrm{NaCl} 505.1870\left([\mathrm{M}+\mathrm{Na}]^{+}\right)$; found 505.1886.

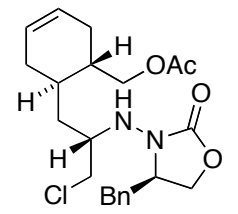

((1S,6R)-6-((S)-2-((R)-4-benzyl-2-oxazolidin-3-ylamino)-3-chloropropyl)cyclohex-3-enyl)methyl acetate (12d). From 8d (85 $\mathrm{mg}, 0.23 \mathrm{mmol})$ and chloroiodomethane $(0.16 \mathrm{~mL}, 2.3 \mathrm{mmol})$ by General Procedure B was obtained 12d (32 mg, 33\% yield) as a colorless oil; $[\alpha]_{\mathrm{D}}{ }^{23}+9.4\left(c 0.16 \mathrm{CHCl}_{3}\right)$; IR (film) $3285,3227,2916,2848,1755,1737,1729,1453,1365,1240,1030 \mathrm{~cm}^{-1} ;{ }^{1} \mathrm{H}$ NMR (300 MHz, $\left.\mathrm{CDCl}_{3}\right) \delta$ 7.35-7.26 (m, 3H), 7.18-7.15 (m, 2H), 5.68-5.58 (m, 2H), 4.18-4.02 $(\mathrm{m}, 5 \mathrm{H}), 3.95-3.86(\mathrm{~m}, 1 \mathrm{H}), 3.65\left(\mathrm{ABX}, \Delta \mathrm{v}_{\mathrm{AB}}=17.9, J_{\mathrm{AB}}=11.4, J_{\mathrm{AX}}=4.4, J_{\mathrm{BX}}=4.2 \mathrm{~Hz}, 2 \mathrm{H}\right)$, $3.43(\mathrm{dd}, J=13.4,3.2 \mathrm{~Hz}, 1 \mathrm{H}), 3.42-3.34(\mathrm{~m}, 1 \mathrm{H}), 2.62(\mathrm{dd}, J=13.2,10.1 \mathrm{~Hz}, 1 \mathrm{H}), 2.24-2.09$ $(\mathrm{m}, 2 \mathrm{H}), 2.08(\mathrm{~s}, 3 \mathrm{H}), 2.08-1.75(\mathrm{~m}, 5 \mathrm{H}), 1.46-1.35(\mathrm{~m}, 1 \mathrm{H}) ;{ }^{13} \mathrm{C} \mathrm{NMR}\left(75 \mathrm{MHz}, \mathrm{CDCl}_{3}\right) \delta$ $171.5,159.5,135.9,129.3,129.2$, 127.4, 125.4, 125.2, 66.61, 66.55, 60.1, 57.9, 46.0, 37.4, 36.2, 34.1, 30.7, 29.3, 26.4, 21.2; MS (ESI) $m / z$ (relative intensity) 421.07 ([M] ${ }^{+}, 53 \%$ ); HRMS (ESI) $\mathrm{m} / z$ calcd. for $\mathrm{C}_{22} \mathrm{H}_{29} \mathrm{~N}_{2} \mathrm{O}_{4} \mathrm{NaCl} 443.1714\left([\mathrm{M}+\mathrm{Na}]^{+}\right)$; found 443.1723. 

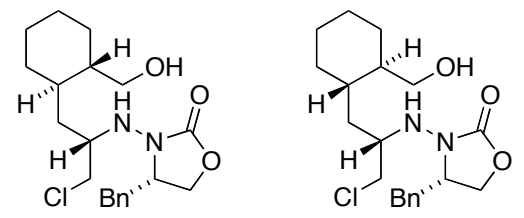

(S)-4-benzyl-3-((S)-3-chloro-1-((1R,2S)-2-(hydroxymethyl)cyclohexyl)propan-2-ylamino)oxazolidin-2-one and $(S)-4-b e n z y l-3-((S)-3-c h l o r o-1-((1 S, 2 R)-2-(h y d r o x y m e t h y l)-$ cyclohexyl)propan-2-ylamino)oxazolidin-2-one (13 and 13'). From 9 (95 mg, $0.37 \mathrm{mmol})$ and iodide $( \pm)-3 c(125 \mathrm{mg}, 0.49$ mmol) by General Procedure B was obtained 13 (95 mg, 67\% yield, dr 1:1); the diastereomers were inseparable, but were separated upon preparation of the $O$-tosylate derivative (see below). Data for diastereomer mixture: IR (film) 3335, 3291, 2921, 2851, 1754, 1402, 1218, $1094 \mathrm{~cm}^{-1}$; ${ }^{1} \mathrm{H}$ NMR $\left(400 \mathrm{MHz}, \mathrm{DMSO}, 70{ }^{\circ} \mathrm{C}\right) \delta$ 7.33-7.29 (m, 2H), 7.24-7.21 (m, 3H), 4.22-4.18 (m, 1H), 4.06-3.98 (m, 2H), 3.75-3.60 (m, 2H), 3.54-3.31 (m, 4H), 3.19-3.14 (m, 1H), 2.75-2.68 (m, 1H), 1.94-1.89 (m, 0.5H), 1.83-1.71 (m, 2H), 1.69-1.60 (m, 2H), 1.60-1.48 (m, 0.5H), 1.33-1.10 (m, 6H), 1.03-0.94 (m, 1H); ${ }^{13} \mathrm{C}$ NMR (100 MHz, DMSO, $\left.70{ }^{\circ} \mathrm{C}\right) \delta 157.2,157.1,136.11,136.09$, 128.8, 128.10, 128.09, 126.1 (2C), 65.1, 65.0, 63.4, 63.2, 58.5, 58.1, 57.3, 57.1, 47.4, 46.5, 44.2, 44.1, 36.1, 36.0, 34.6, 34.1, 34.0, 33.8, 31.5, 31.2, 28.8, 28.6, 25.0, 24.9 (2C), 24.7; MS (ESI) $\mathrm{m} / \mathrm{z}$ (relative intensity) $381.10\left([\mathrm{M}+\mathrm{H}]^{+} 81 \%\right), 403.16\left([\mathrm{M}+\mathrm{Na}]^{+}, 100 \%\right)$; Anal. Calcd for $\mathrm{C}_{20} \mathrm{H}_{29} \mathrm{ClN}_{2} \mathrm{O}_{3} ; \mathrm{C}, 63.06 ; \mathrm{H}, 7.67 ; \mathrm{N}, 7.35$. Found: C, 62.87; H, 7.70; N, 7.33.
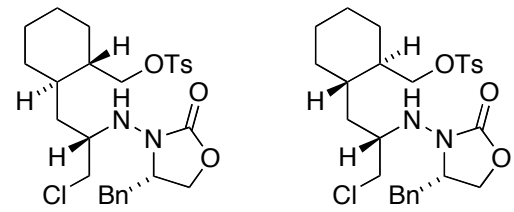

$((1 S, 2 R)-2-((S)-2-((S)-4-b e n z y l-2-o x a z o l i d i n-3-y l a m i n o)-3-$ chloropropyl)cyclohexyl)methyl 4-methylbenzenesulfonate and $((1 R, 2 S)-2-((S)-2-((S)-4-b e n z y l-2-o x a z o l i d i n-3-y l a m i n o)-$ 3-chloropropyl)cyclohexyl)methyl 4-methylbenzenesulfonate (S7 and S7'). To a solution of $\mathbf{1 3}$ ( $\mathrm{dr} 1: 1,70 \mathrm{mg}, 0.18 \mathrm{mmol})$, triethylamine $(0.1 \mathrm{~mL}, 0.27 \mathrm{mmol})$, and DMAP $(2 \mathrm{mg}, 0.02 \mathrm{mmol})$ in $\mathrm{CH}_{2} \mathrm{Cl}_{2}(2 \mathrm{~mL})$ was added p-toluenesulfonyl chloride $(42 \mathrm{mg}, 0.22 \mathrm{mmol}$ ) at room temperature. After $4 \mathrm{~h}$, the reaction mixture was partitioned between $\mathrm{CH}_{2} \mathrm{Cl}_{2}$ and brine, dried $\left(\mathrm{Na}_{2} \mathrm{SO}_{4}\right)$, and concentrated. Flash chromatography (hexane/EtOAc (9:1 to 2:1) afforded the separate diastereomers $\mathbf{S 7}$ and $\mathbf{S 7}$ ' (1:1 ratio, $80 \mathrm{mg}, 81 \%$ yield). Relative configuration was not determined. More polar diastereomer S7: $\mathrm{R}_{\mathrm{f}} 0.5\left(\mathrm{SiO}_{2}, 30 \% \mathrm{EtOAc} /\right.$ hexanes $) ;[\alpha]_{\mathrm{D}}{ }^{25}+24.4\left(c 1.60 \mathrm{CHCl}_{3}\right)$; IR (film) $3295,2925,2855$, $1755,1598,1448,1400,1358,1175,1096,1028 \mathrm{~cm}^{-1} ;{ }^{1} \mathrm{H}$ NMR $\left(300 \mathrm{MHz}, \mathrm{CDCl}_{3}\right) \delta 7.78(\mathrm{~d}, J=$ 8.4Hz, 2H), 7.34-7.23(m, 5H), 7.19-7.16 (m, 2H), 4.18-3.93 (m, 5H), $3.65(\mathrm{dd}, J=11.7,3.6 \mathrm{~Hz}$, $1 \mathrm{H}), 3.49$ (dd, $J=11.7,3.9 \mathrm{~Hz}, 1 \mathrm{H}), 3.37-3.32(\mathrm{~m}, 2 \mathrm{H}), 2.58(\mathrm{dd}, J=13.3,10.2 \mathrm{~Hz}, 1 \mathrm{H}), 2.43(\mathrm{~s}$, $3 \mathrm{H}), 1.83-1.68(\mathrm{~m}, 4 \mathrm{H}), 1.38-1.11(\mathrm{~m}, 8 \mathrm{H}), 1.10-0.90(\mathrm{~m}, 1 \mathrm{H}) ;{ }^{13} \mathrm{C}$ NMR $\left(75 \mathrm{MHz}, \mathrm{CDCl}_{3}\right) \delta$ 158.9, 145.0, 135.9, 133.1, 130.0, 129.5, 129.1, 128.1, 127.3, 73.0, 68.2, 59.9, 58.0, 45.9, 42.1, 37.1, 35.1, 34.3, 32.2, 29.5, 25.6, 25.5, 21.9; MS (ESI) $\mathrm{m} / z$ (relative intensity) $557.10\left([\mathrm{M}+\mathrm{Na}]^{+}\right.$ $100 \%), 535.06\left([\mathrm{M}+\mathrm{H}]^{+}, 47 \%\right)$. Less polar diastereomer $\mathbf{S 7}$ ': $\mathrm{R}_{\mathrm{f}} 0.6\left(\mathrm{SiO}_{2}, 30 \%\right.$ EtOAc/hexanes); $[\alpha]_{\mathrm{D}}{ }^{25}-25.3$ (c $1.62 \mathrm{CHCl}_{3}$ ); IR (film) 3318, 2924, 2854, 1755, 1598, 1448, 1400, 1358, 1188, 1175, 1097, $1029 \mathrm{~cm}^{-1} ;{ }^{1} \mathrm{H}$ NMR $\left(300 \mathrm{MHz}, \mathrm{CDCl}_{3}\right) \delta 7.79(\mathrm{~d}, J=8.3 \mathrm{~Hz}, 2 \mathrm{H})$, 7.36-7.23(m, 5H), 7.20-7.17 (m, 2H), 4.15-3.94 (m, 6H), $3.61(\mathrm{dd}, J=11.3,3.6 \mathrm{~Hz}, 1 \mathrm{H}), 3.49$ $(\mathrm{dd}, J=11.2,6.0 \mathrm{~Hz}, 1 \mathrm{H}), 3.34(\mathrm{dd}, J=13.4,3.3 \mathrm{~Hz}, 1 \mathrm{H}), 3.36-3.25(\mathrm{~m}, 1 \mathrm{H}), 2.60$ (dd, $J=13.3$, $10.0 \mathrm{~Hz}, 1 \mathrm{H}), 2.44$ (s, 3H), 2.04-1.98 (m, 1H), 1.75-1.49 (m, 5H), 1.36-1.18 (m, 6H), 1.03-0.90 $(\mathrm{m}, 1 \mathrm{H}) ;{ }^{13} \mathrm{C} \mathrm{NMR}\left(75 \mathrm{MHz}, \mathrm{CDCl}_{3}\right) \delta 159.2,145.1,135.9,133.0,130.1,129.3,129.2,128.1$, 127.3, 72.8, 66.2, 60.5, 58.5, 47.4, 42.3, 37.2, 34.6, 34.5, 31.6, 29.6, 25.7, 25.6, 21.9; MS (ESI) $\mathrm{m} / \mathrm{z}$ (relative intensity) $557.11\left([\mathrm{M}+\mathrm{Na}]^{+} 100 \%\right), 535.06\left([\mathrm{M}+\mathrm{H}]^{+}, 51 \%\right)$. 


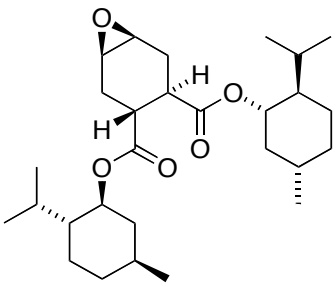

$(1 S, 3 R, 4 R, 6 R)-3-((1 S, 2 R, 5 S)-2$-isopropyl-5-methylcyclohexyl) 4 $((1 S, 2 R, 5 S)$-2-isopropyl-5-methylcyclohexyl) 7-oxa-bicyclo[4.1.0]heptane-3,4-dicarboxylate (S8). To a solution of $\mathbf{1}(13.4 \mathrm{~g}, 30.2 \mathrm{mmol})$ in $\mathrm{CH}_{2} \mathrm{Cl}_{2}(200 \mathrm{~mL})$ was added $m$-chloroperbenzoic acid $(50-60 \%, 15.6 \mathrm{~g}$, $45.3 \mathrm{mmol}$ ). After $3 \mathrm{~h}$, the reaction mixture was diluted with $\mathrm{CH}_{2} \mathrm{Cl}_{2}$, washed successively with saturated aqueous $\mathrm{NaHCO}_{3}$ and brine, dried $\left(\mathrm{Na}_{2} \mathrm{SO}_{4}\right)$, and concentrated. Flash chromatography (hexane/EtOAc 20:1 to 4:1) afforded $\mathbf{S 8}$ (12 g, 86\% yield) as a colorless oil; $[\alpha]_{\mathrm{D}}{ }^{24}+41.6\left(c 2.88 \mathrm{CHCl}_{3}\right)$; IR (film) 2954, 2869, 1937, 1726, 1453, 1387, 1370, 1307, 1242, 1171, $1021 \mathrm{~cm}^{-1} ;{ }^{1} \mathrm{H}$ NMR (300 MHz, $\mathrm{CDCl}_{3}$ ) ठ 4.67-4.57 (m, $2 \mathrm{H}), 3.22-3.17(\mathrm{~m}, 1 \mathrm{H}), 3.16(\mathrm{dd}, J=4.5,3.9 \mathrm{~Hz}, 1 \mathrm{H}), 2.80(\mathrm{ddd}, J=10.4,10.4,4.9 \mathrm{~Hz}, 1 \mathrm{H})$, 2.59 (ddd, $J=10.4,10.4,6.7,1 \mathrm{H}), 2.45$ (ddd, $J=14.7,4.8,1.7 \mathrm{~Hz}, 1 \mathrm{H}), 2.29$ (ddd, $J=15.4,6.6$, $4.7 \mathrm{~Hz}, 1 \mathrm{H},), 2.06-1.79(\mathrm{~m}, 6 \mathrm{H}), 1.66-1.62(\mathrm{~m}, 4 \mathrm{H}), 1.52-1.31(\mathrm{~m}, 4 \mathrm{H}), 1.08-0.81(\mathrm{~m}, 18 \mathrm{H})$, 0.73-0.69 (m, 6H) ${ }^{13} \mathrm{C}$ NMR (75 MHz, $\left.\mathrm{CDCl}_{3}\right) \delta$ 174.5, 173.5, 74.7 (2C), 52.0, 50.5, 47.1, 47.1, 40.8 (2C), 40.3, 37.9, 34.4, 34.4, 31.6, 31.5, 27.4, 26.6, 26.2, 26.1, 23.3 (2C), 22.2 (2C), 21.0, 21.0, 16.10, 16.08; MS (ESI) $\mathrm{m} / z$ (relative intensity) $485.25\left([\mathrm{M}+\mathrm{Na}]^{+} 100 \%\right), 947.3\left([2 \mathrm{M}+\mathrm{Na}]^{+}\right.$, $68 \%$ ); Anal. Calcd for $\mathrm{C}_{28} \mathrm{H}_{46} \mathrm{O}_{5}$ : C, 72.69; H, 10.02; Found: C, 72.91; H, 10.12.

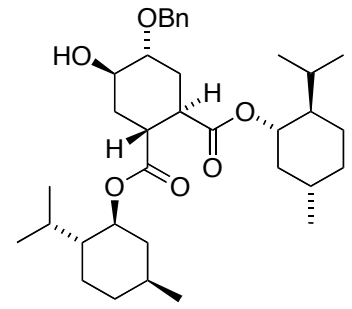

$(1 R, 2 R, 4 R, 5 R)$-bis $((1 S, 2 R, 5 S)$-2-isopropyl-5-methylcyclohexyl) 4(benzyloxy)-5-hydroxycyclohexane-1,2-dicarboxylate (S9). To a solution of $\mathbf{S 8}(11 \mathrm{~g}, 23.8 \mathrm{mmol})$ and benzyl alcohol $(12.8 \mathrm{~g}, 119 \mathrm{mmol})$ was added $\mathrm{Cu}(\mathrm{OTf})_{2}(1.72 \mathrm{~g}, 4.76 \mathrm{mmol})$. After $1 \mathrm{~d}$, the reaction mixture was diluted with $\mathrm{CH}_{2} \mathrm{Cl}_{2}$, washed successively with water and brine, dried $\left(\mathrm{Na}_{2} \mathrm{SO}_{4}\right)$, and concentrated. Flash chromatography (hexane/EtOAc 9:1 to 4:1) provided $\mathbf{S 9}(12.1 \mathrm{~g}, 89 \%$ yield $)$ as a colorless solid; mp $103-104{ }^{\circ} \mathrm{C} ;[\alpha]_{\mathrm{D}}{ }^{26}+48.7(c 1.5$, $\mathrm{CHCl}_{3}$ ); IR (film) 3491, 2955, 2927, 2869, 1736, 1726, 1710, 1452, 1369, 1241, 1165, 1071, $1028 \mathrm{~cm}^{-1}$; ${ }^{1} \mathrm{H}$ NMR $\left(300 \mathrm{MHz}, \mathrm{CDCl}_{3}\right) \delta$ 7.36-7.27 (m, 5H), 4.72-4.62 (m, 3H), $4.50(\mathrm{~d}, J=$ $11.8 \mathrm{~Hz}, 1 \mathrm{H}), 3.89-3.82(\mathrm{~m}, 1 \mathrm{H}), 3.51-3.46(\mathrm{~m}, 1 \mathrm{H}), 3.12-3.05(\mathrm{~m}, 2 \mathrm{H}), 2.20-1.80(9 \mathrm{H}), 1.69-$ $1.65(\mathrm{~m}, 2 \mathrm{H}), 1.55-1.39(\mathrm{~m}, 4 \mathrm{H}), 1.10-0.84(\mathrm{~m}, 20 \mathrm{H}), 0.75-0.71(\mathrm{~m}, 6 \mathrm{H}) ;{ }^{13} \mathrm{C}$ NMR $(75 \mathrm{MHz}$, $\left.\mathrm{CDCl}_{3}\right) \delta$ 174.1, 174.0, 138.5, 128.7, 128.0, 127.8, 77.2, 74.69, 74.68, 71.2, 68.3, 47.1, 47.1, 40.90, 40.88, 40.0, 39.6, 34.4 (2C), 31.6 (2C), 30.9, 27.4, 26.2, 26.1, 23.3, 23.2, 22.2 (2C), 21.09, 21.07, 16.2, 16.1; MS (EI) $\mathrm{m} / z$ (relative intensity) 570.47 ([M] $2 \%$ ) ; Anal. Calcd for $\mathrm{C}_{35} \mathrm{H}_{54} \mathrm{O}_{6}$; C, 73.65; H, 9.54; Found: C, 73.46; H, 9.45. Crystallography confirmed the structure.

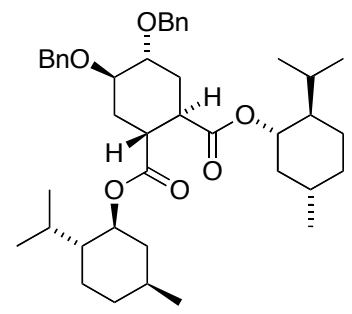

$(1 R, 2 R, 4 R, 5 R)-b i s((1 S, 2 R, 5 S)$-2-isopropyl-5-methylcyclohexyl) 4,5bis(benzyloxy)cyclohexane-1,2-dicarboxylate (14). To a solution of $\mathbf{S 9}$ $(12.1 \mathrm{~g}, 21.12 \mathrm{mmol})$ and benzyl trichloroacetamidate $(8.45 \mathrm{~g}, 42.2 \mathrm{mmol})$ in $\mathrm{CH}_{2} \mathrm{Cl}_{2}(30 \mathrm{~mL})$ and cyclohexane $(60 \mathrm{~mL})$ was added trifluoromethanesulfonic acid $(0.17 \mathrm{~mL}, 2 \mathrm{mmol})$ at room temperature. After $1 \mathrm{~d}$, the reaction mixture was diluted with $\mathrm{CH}_{2} \mathrm{Cl}_{2}$, washed successively with saturated aqueous $\mathrm{NaHCO}_{3}$ solution and brine, dried $\left(\mathrm{Na}_{2} \mathrm{SO}_{4}\right)$ and concentrated. Flash chromatography (hexane/EtOAc 30:1 to 9:1) afforded 14 (14 $\mathrm{g},>99 \%$ yield) as a colorless liquid; $[\alpha]_{\mathrm{D}}{ }^{24}+44.4\left(c 1.83, \mathrm{CHCl}_{3}\right)$; IR (film) $2954,2925,2864$, $1726,1450,1368,1249,1155,1086 \mathrm{~cm}^{-1}$; ${ }^{1} \mathrm{H}$ NMR $\left(300 \mathrm{MHz}, \mathrm{CDCl}_{3}\right) \delta$ 7.31-7.18 (m, 10H), 4.63-4.41 (m, 6H), 3.60 (s, 2H), 3.08-2.86 (m, 2H), 2.05-1.75 (m, 8H), 1.62-1.57 (m, 4H), 1.47$1.28(\mathrm{~m}, 4 \mathrm{H}), 1.04-0.78(\mathrm{~m}, 18 \mathrm{H}), 0.69-0.65(\mathrm{~m}, 3 \mathrm{H}) ;{ }^{13} \mathrm{C} \mathrm{NMR}\left(75 \mathrm{MHz}, \mathrm{CDCl}_{3}\right) \delta 174.6$, 138.6, 128.6, 127.8, 127.7, 74.4, 73.9, 71.1, 47.1, 40.9, 39.5, 34.5, 31.6, 28.0, 26.2, 23.3, 22.2, 
21.1, 16.2; MS (ESI) $\mathrm{m} / z$ (relative intensity) $661.11\left([\mathrm{M}+\mathrm{H}]^{+} 17 \%\right), 683.41\left([\mathrm{M}+\mathrm{Na}]^{+}, 100 \%\right)$; Anal. Calcd for $\mathrm{C}_{42} \mathrm{H}_{60} \mathrm{O}_{6}$; C, 76.33; H, 9.54; Found: C, 76.16; H, 9.20.

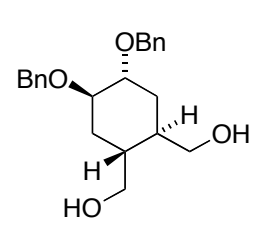

$((1 R, 2 R, 4 R, 5 R)-4,5-b i s($ benzyloxy)-2-(hydroxymethyl)cyclohexyl)methanol (S10). A solution of the diester $\mathbf{1 4}(14 \mathrm{~g}, 21 \mathrm{mmol})$ in ether $(40 \mathrm{~mL})$ was added dropwise to a mixture of lithium aluminum hydride $(1.66 \mathrm{~g}, 45 \mathrm{mmol})$ in ether $(60 \mathrm{~mL})$ at $-78{ }^{\circ} \mathrm{C}$. The mixture was allowed to warm to room temperature over $12 \mathrm{~h}$, then was cooled to $-20{ }^{\circ} \mathrm{C}$ and quenched by successive addition of water $(3 \mathrm{~mL})$, aqueous $\mathrm{NaOH}(3 \mathrm{M}, 3 \mathrm{~mL})$, and water $(6 \mathrm{~mL})$. Filtration through celite, concentration, and flash chromatography (hexane/EtOAc 3:1 to 1:2) furnished $\mathbf{S 1 0}$ (6.43 $\mathrm{g}, 85 \%$ yield) as a colorless liquid; $[\alpha]_{\mathrm{D}}{ }^{23}-20.7$ (c 1.14, $\mathrm{CHCl}_{3}$ ); IR (film) 3353, 3029, 2911, 2877, 1452, 1432, 1394, 1352, 1091, 1065, $1027 \mathrm{~cm}^{-1},{ }^{1} \mathrm{H}$ NMR (300 MHz, $\left.\mathrm{CDCl}_{3}\right) \delta$ 7.30-7.18 $(\mathrm{m}, 10 \mathrm{H}), 4.45\left(\mathrm{ABq}, \Delta v_{\mathrm{AB}}=24.5, J_{\mathrm{AB}}=12.0,4 \mathrm{H}\right), 3.99(\mathrm{br} \mathrm{s}, 2 \mathrm{H}), 3.64-3.60(\mathrm{~m}, 2 \mathrm{H}), 3.49-3.39$ (m, 4H), 1.70-1.42 (m, 6H); ${ }^{13} \mathrm{C}$ NMR $\left(75 \mathrm{MHz}, \mathrm{CDCl}_{3}\right) \delta$ 138.7, 128.5, 127.7, 127.6, 74.5, 70.8, 67.4, 38.5, 28.8; MS (ESI) $\mathrm{m} / z$ (relative intensity) 356.85 ([M] ${ }^{+} 84 \%$ ); Anal. Calcd for $\mathrm{C}_{22} \mathrm{H}_{28} \mathrm{O}_{4}$; C, 74.13; H, 7.92; Found: C, 74.34; H, 8.10.

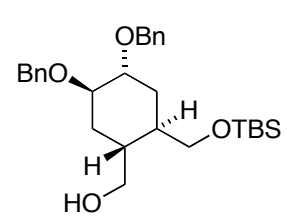

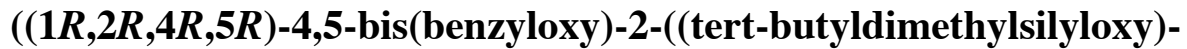
methyl)cyclohexyl)methanol (15). To a solution of diol S10 (6.35 g, 17.83 $\mathrm{mmol})$ in THF $(120 \mathrm{~mL})$ was added $n$-BuLi $(1.5 \mathrm{M}$ in hexane, $14.2 \mathrm{~mL}, 21.4$ $\mathrm{mmol})$ at $0{ }^{\circ} \mathrm{C}$. After warming to room temperature for 80 min under $\mathrm{N}_{2}$, tertbutyldimethylsilyl chloride ( $2.68 \mathrm{~g}, 17.83 \mathrm{mmol})$ was added. After ca. $12 \mathrm{~h}$, the reaction mixture was partitioned between ethyl acetate and saturated $\mathrm{NaHCO}_{3}$. The aqueous layer was extracted with $\mathrm{CH}_{2} \mathrm{Cl}_{2}(3 \times 15 \mathrm{~mL})$. The combined organic phase was dried $\left(\mathrm{Na}_{2} \mathrm{SO}_{4}\right)$ and concentrated. Flash chromatography (hexane/EtOAc 9:1 to 4:1) afforded alcohol 15 (7.86 g, 93\% yield) as colorless liquid; $[\alpha]_{\mathrm{D}}{ }^{25}-14.4\left(c \mathrm{1.55}, \mathrm{CHCl}_{3}\right.$ ); IR (film) 3437, 3029, 2952, 2927 , 2856, 1461, 1389, 1360, 1253, 1094, $1072 \mathrm{~cm}^{-1}$; ${ }^{1} \mathrm{H}$ NMR $\left(300 \mathrm{MHz}, \mathrm{CDCl}_{3}\right) \delta$ 7.30-7.18 (m, $10 \mathrm{H}), 4.54-4.37(\mathrm{~m}, 4 \mathrm{H}), 3.65-3.61(\mathrm{~m}, 2 \mathrm{H}), 3.53-3.44(\mathrm{~m}, 5 \mathrm{H}), 1.75-1.48(\mathrm{~m}, 6 \mathrm{H}), 0.86(\mathrm{~s}, 9 \mathrm{H})$, $0.04(\mathrm{~s}, 6 \mathrm{H}) ;{ }^{13} \mathrm{C}$ NMR $\left(75 \mathrm{MHz}, \mathrm{CDCl}_{3}\right) \delta 138.92,138.91,128.51,128.48,127.7,127.61$, 127.55, 127.5, 74.8, 74.7, 70.8, 70.7, 68.3, 67.2, 38.9, 37.8, 28.9 (2C), 26.0, 18.3, -5.2, -5.4; MS (ESI) $\mathrm{m} / z$ (relative intensity) $470.99\left(\mathrm{M}^{+}, 91 \%\right)$; Anal. Calcd for $\mathrm{C}_{28} \mathrm{H}_{42} \mathrm{O}_{4} \mathrm{Si}$; C, 71.44; H, 8.99; Found: C, 71.70; H, 9.12.

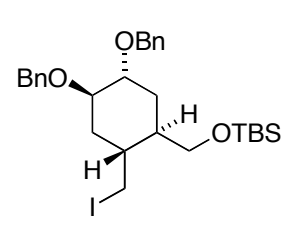

$((1 R, 2 R, 4 R, 5 R)-4,5$-bis(benzyloxy)-2-(iodomethyl)cyclohexyl)methoxy)(tert-butyl)dimethylsilane (S11). To a solution of alcohol $15(575 \mathrm{mg}$, $1.22 \mathrm{mmol})$ in THF $(6 \mathrm{~mL})$ was added imidazole $(210 \mathrm{mg}, 3.05 \mathrm{mmol})$ and triphenylphosphine $(640 \mathrm{mg}, 2.44 \mathrm{mmol})$. After $10 \mathrm{~min}$, iodine $(620 \mathrm{mg}, 2.44$ mmol) was added in two portions over $10 \mathrm{~min}$. After $15 \mathrm{~min}$, the reaction mixture was partitioned between EtOAc and saturated aqueous $\mathrm{Na}_{2} \mathrm{~S}_{2} \mathrm{O}_{3}$. The aqueous phase was extracted with $\mathrm{CH}_{2} \mathrm{Cl}_{2}(3 \times 10 \mathrm{~mL})$. The combined organic phase was dried $\left(\mathrm{Na}_{2} \mathrm{SO}_{4}\right)$ and concentrated. Flash chromatography (hexane to hexane/EtOAc 9:1) gave iodide $\mathbf{S 1 1}$ (580 $\mathrm{mg}$, $82.4 \%$ yield) as a colorless oil; $[\alpha]_{\mathrm{D}}{ }^{23}-38.5\left(c 1.57, \mathrm{CHCl}_{3}\right)$; IR (film) 3024, 2952, 2926, 2884, 2885, 1469, 1462, 1252, $1107 \mathrm{~cm}^{-1} ;{ }^{1} \mathrm{H}$ NMR (300 MHz, $\left.\mathrm{CDCl}_{3}\right) \delta$ 7.28-7.16 (m, 10H), 4.52 (d, $J$ $=12.0 \mathrm{~Hz}, 1 \mathrm{H}), 4.51(\mathrm{~d}, J=12.0 \mathrm{~Hz}, 1 \mathrm{H}), 4.41(\mathrm{~d}, J=12.0 \mathrm{~Hz}, 1 \mathrm{H}), 4.39(\mathrm{~d}, J=12.0 \mathrm{~Hz}, 1 \mathrm{H})$, $3.65-3.60(\mathrm{~m}, 2 \mathrm{H}), 3.57(\mathrm{dd}, J=10.2,4.4 \mathrm{~Hz}, 1 \mathrm{H}), 3.41(\mathrm{dd}, J=10.2,3.2 \mathrm{~Hz}, 1 \mathrm{H}), 3.33(\mathrm{dd}, J=$ 9.9, $2.7 \mathrm{~Hz}, 1 \mathrm{H}), 3.22(\mathrm{dd}, J=9.8,5.8 \mathrm{~Hz}, 1 \mathrm{H}), 1.83-1.50(\mathrm{~m}, 6 \mathrm{H}), 0.83(\mathrm{~s}, 9 \mathrm{H}), 0.0(\mathrm{~s}, 6 \mathrm{H}) ;{ }^{13} \mathrm{C}$ 
NMR $\left(75 \mathrm{MHz}, \mathrm{CDCl}_{3}\right) \delta 139.03,138.96,128.53,128.51,127.6,127.53,127.52$ (2C), 74.8 (2C), 70.7, 70.6, 64.8, 38.0, 33.3, 32.2, 28.4, 26.1, 18.4, 16.5, -5.2, -5.3; MS (EI) $\mathrm{m} / z$ (relative intensity); $523\left([\mathrm{M}-t-\mathrm{Bu}]^{+},<1 \%\right)$.

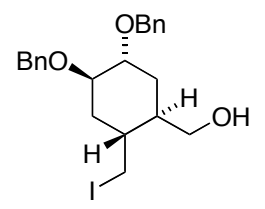

((1R,2R,4R,5R)-4,5-bis(benzyloxy)-2-(iodomethyl)cyclohexyl)methanol (3d). A solution of $\mathbf{S 1 1}$ (580 $\mathrm{mg}, 1 \mathrm{mmol}$ ) and camphorsulfonic acid (20 mg, $0.1 \mathrm{mmol})$ in methanol $(6 \mathrm{~mL})$ was stirred for $4 \mathrm{~h}$ at room temperature. Concentration and flash chromatography (hexane/EtOAc 9:1 to 2:1) afforded 3d (400 mg, 85\% yield) as a colorless oil; $[\alpha]_{\mathrm{D}}{ }^{24}-32.2\left(c 0.62, \mathrm{CHCl}_{3}\right)$; IR (film) 3425, 3028, 2917, 2874, 1452, 1325, 1179, 1090, 1072, $1027 \mathrm{~cm}^{-1} ;{ }^{1} \mathrm{H}$ NMR $\left(300 \mathrm{MHz}, \mathrm{CDCl}_{3}\right)$ $\delta 7.30-7.16(\mathrm{~m}, 10 \mathrm{H}), 4.50(\mathrm{~d}, J=11.8 \mathrm{~Hz}, 2 \mathrm{H}), 4.42(\mathrm{~d}, J=12.3 \mathrm{~Hz}, 1 \mathrm{H}), 4.40(\mathrm{~d}, J=12.0 \mathrm{~Hz}$, $1 \mathrm{H}), 3.65-3.61(\mathrm{~m}, 2 \mathrm{H}), 3.57-3.47(\mathrm{~m}, 2 \mathrm{H}), 3.34-3.24(\mathrm{~m}, 2 \mathrm{H}), 1.82-1.39(\mathrm{~m}, 6 \mathrm{H}) ;{ }^{13} \mathrm{C}$ NMR $(75$ $\left.\mathrm{MHz}, \mathrm{CDCl}_{3}\right) \delta 138.9,138.8,128.6,128.5,127.8,127.7$ (2C), 127.5, 74.60, 74.57, 70.9, 70.8, 65.2, 38.0, 33.6, 32.4, 28.2, 16.4; MS (EI) $\mathrm{m} / \mathrm{z}$ (relative intensity); 338 ([M-I] $]^{+},<1 \%$ ), 247 $\left([\mathrm{M}-\mathrm{I}-\mathrm{Bn}]^{+}, 92 \%\right)$.

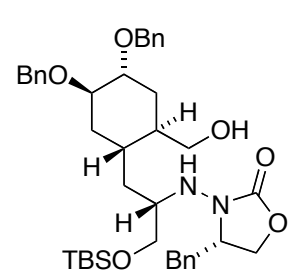

$(S)-3-((S)-1-((1 R, 2 R, 4 R, 5 R)-4,5-b i s(b e n z y l o x y)-2-(h y d r o x y m e t h y l)-$ cyclohexyl)-3-(tert-butyldimethylsilyloxy)propan-2-ylamino)-4benzyloxa-zolidin-2-one (17). From (S)-4-benzyl-3-(2-(tertbutyldimethylsilyloxy)-ethylideneamino)oxazolidin-2-one (356 mg, 1.02 mmol) and iodide 3d (600 mg, $1.28 \mathrm{mmol})$ by General Procedure B was obtained $17(658 \mathrm{mg}, 93 \%$ yield $)$ as a colorless oil; $[\alpha]_{\mathrm{D}}{ }^{24}-13.4(c \quad 0.29$, $\mathrm{CHCl}_{3}$ ); IR (film) 3474, 3028, 2926, 2856, 1775, 1764, 1452, 1251, 1094, $1027 \mathrm{~cm}^{-1} ;{ }^{1} \mathrm{H} \mathrm{NMR}$ $\left(300 \mathrm{MHz}, \mathrm{CDCl}_{3}\right) \delta$ 7.34-7.02 (m, 15H), 4.54-4.34 (m, 5H), 3.98-3.74 (m, 4H), 3.67-3.45 (m, $5 \mathrm{H}), 3.26(\mathrm{dd}, J=12.9,1.9 \mathrm{~Hz}, 1 \mathrm{H}), 3.15-3.05(\mathrm{~m}, 1 \mathrm{H}), 2.55-2.40(\mathrm{~m}, 1 \mathrm{H}), 2.00-1.69(\mathrm{~m}, 5 \mathrm{H})$, 1.55-1.47 (m, 2H), 1.21-1.06 (m, 1H), $0.86(\mathrm{~s}, 9 \mathrm{H}), 0.04(\mathrm{~s}, 3 \mathrm{H}), 0.03(\mathrm{~s}, 3 \mathrm{H}) ;{ }^{13} \mathrm{C}$ NMR $(75$ $\left.\mathrm{MHz}, \mathrm{CDCl}_{3}\right) \delta 159.0,139.02,138.95,135.8,129.2,129.2,129.1,128.4,127.62,127.55$, 127.53, 127.49, 127.2, 74.9 (2C), 70.90, 70.87, 66.1, 65.4, 64.7, 59.0, 58.9, 39.3, 37.3, 34.6, 32.1, 29.4, 28.9, 27.1, 18.5, -5.1, -5.2; MS (ESI) $\mathrm{m} / \mathrm{z}$ (relative intensity) $689.25\left([\mathrm{M}+1]^{+} 100 \%\right)$; Anal. Calcd for $\mathrm{C}_{40} \mathrm{H}_{56} \mathrm{~N}_{2} \mathrm{O}_{6} \mathrm{Si}$; C, 69.73; H, 8.19; N, 4.07; Found: C, 69.99; H, 8.13; N, 3.97.

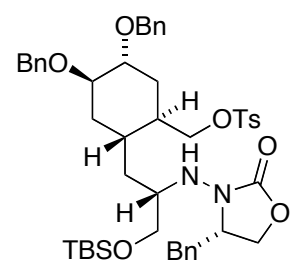

$((1 R, 2 R, 4 R, 5 R)-2-((S)-2-((S)-4-b e n z y l-2-0 x 0 o x a z o l i d i n-3-y l a m i n o)-3-$ (tert-butyldimethylsilyloxy)propyl)-4,5-bis(benzyloxy)cyclohexyl)methyl 4-methylbenzenesulfonate (S12). To a solution of 17 (195 mg, $0.28 \mathrm{mmol})$, triethylamine $(0.1 \mathrm{~mL}, 0.52 \mathrm{mmol})$, 4-dimethylaminopyridine $(7 \mathrm{mg}, 0.06$ mmol) in $\mathrm{CH}_{2} \mathrm{Cl}_{2}(2 \mathrm{~mL}$ ) was added $p$-toluenesulfonyl chloride (64 mg, 0.33 $\mathrm{mmol}$ ) at room temperature. After $4 \mathrm{~h}$, the reaction mixture was diluted with $\mathrm{CH}_{2} \mathrm{Cl}_{2}$, washed with brine, dried $\left(\mathrm{Na}_{2} \mathrm{SO}_{4}\right)$ and concentrated. Flash chromatography (hexane/EtOAc 9:1 to 2:1) gave $\mathbf{S 1 2}$ (223 mg, 94\% yield) as a colorless oil; $[\alpha]_{\mathrm{D}}{ }^{23}-17.5$ (c 3.8, $\mathrm{CHCl}_{3}$ ); IR (film) 3295, 3062, 3029, 2927, 2856, 1775, 1764, 1758, 1755, 1494, 1452, 1399, 1358, 1250, 1176, $1096 \mathrm{~cm}^{-1}$; ${ }^{1} \mathrm{H}$ NMR $\left(300 \mathrm{MHz}, \mathrm{CDCl}_{3}\right) \delta 7.78(\mathrm{~d}, J=8.3 \mathrm{~Hz}, 2 \mathrm{H}), 7.31-7.23$ $(\mathrm{m}, 15 \mathrm{H}), 7.16-7.13(\mathrm{~m}, 2 \mathrm{H}), 4.60(\mathrm{~d}, J=12.3 \mathrm{~Hz}, 1 \mathrm{H}), 4.53-4.41(\mathrm{~m}, 3 \mathrm{H}), 4.12-3.94(\mathrm{~m}, 6 \mathrm{H})$, $3.67-3.63(\mathrm{~m}, 3 \mathrm{H}), 3.53(\mathrm{dd}, J=10.0,5.4 \mathrm{~Hz}, 1 \mathrm{H}), 3.31(\mathrm{dd}, J=13.2,2.5 \mathrm{~Hz}, 1 \mathrm{H}), 3.25-3.15(\mathrm{~m}$, $1 \mathrm{H}), 2.60(\mathrm{dd}, J=13.2,9.1 \mathrm{~Hz}, 1 \mathrm{H}), 2.42$ (s, 3H), 2.10 (br d, $13.5 \mathrm{~Hz}, 1 \mathrm{H}), 1.89-1.69(\mathrm{~m}, 4 \mathrm{H})$, $1.61-1.43(\mathrm{~m}, 2 \mathrm{H}), 1.29-1.20(\mathrm{~m}, 1 \mathrm{H}), 0.91(\mathrm{~s}, 9 \mathrm{H}), 0.08(\mathrm{~s}, 3 \mathrm{H}), 0.07(\mathrm{~s}, 3 \mathrm{H}) ;{ }^{13} \mathrm{C}$ NMR $(75$ $\left.\mathrm{MHz}, \mathrm{CDCl}_{3}\right) \delta 158.8,144.8,138.8,138.7,135.9,133.1,130.0,129.2,129.0,128.9,128.5$, 
128.4, 128.0, 127.7, 127.6, 127.5, 127.1, 74.3, 74.0, 72.7, 70.8, 70.7, 65.9, 65.3, 59.5, 58.4, 37.0, 36.9, 33.7, 30.7, 28.9, 28.8, 26.1, 21.7, 18.4, -5.22, -5.24; MS (ESI) $\mathrm{m} / z$ (relative intensity) $843.28\left([\mathrm{M}]^{+} 100 \%\right)$; Anal. Calcd for $\mathrm{C}_{47} \mathrm{H}_{62} \mathrm{~N}_{2} \mathrm{O}_{8} \mathrm{SSi}$; C, 66.95; H, 7.41; N, 3.32; Found: C, 67.09; H, 7.29; N, 3.34.

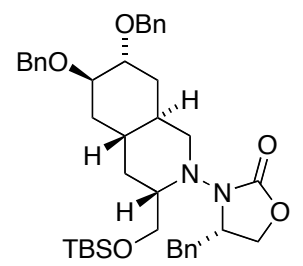

(S)-3-((3S,4aR,6R,7R,8aR)-6,7-bis(benzyloxy)-3-((tert-butyldimethylsilyloxy)methyl)-octahydroisoquinolin-2(1H)-yl)-4-benzyloxazolidin-2one (18). To a solution of $\mathbf{S 1 2}(42 \mathrm{mg}, 0.049 \mathrm{mmol})$ in acetonitrile was added $\mathrm{K}_{2} \mathrm{CO}_{3}(67 \mathrm{mg}, 0.49 \mathrm{mmol})$ and $\mathrm{NaI}(15 \mathrm{mg}, 0.1 \mathrm{mmol})$ and the mixture was heated under reflux for $6 \mathrm{~h}$. The reaction mixture was diluted with ethyl acetate, washed successively with water and brine, dried $\left(\mathrm{Na}_{2} \mathrm{SO}_{4}\right)$ and concentrated. Flash chromatography (hexane/EtOAc 20:1 to 9:1) afforded 18 (27 mg, 82\% yield) as a colorless oil; $[\alpha]_{\mathrm{D}}{ }^{23}-21.6\left(c 0.97, \mathrm{CHCl}_{3}\right)$; IR (film) 2926, 2855, 1755, 1453, 1399 , 1251, 1091, 1071, $1027 \mathrm{~cm}^{-1} ;{ }^{1} \mathrm{H}$ NMR $\left(600 \mathrm{MHz}\right.$, acetone- $\left.d_{6}\right) \delta$ 7.36-7.19 (m, 15H), $4.61(\mathrm{~d}, J=$ $12.1 \mathrm{~Hz}, 1 \mathrm{H}), 4.60(\mathrm{~d}, J=12.0 \mathrm{~Hz}, 1 \mathrm{H}), 4.54(\mathrm{~d}, J=12.0 \mathrm{~Hz}, 1 \mathrm{H}), 4.53(\mathrm{~d}, J=12.0 \mathrm{~Hz}, 1 \mathrm{H})$, $4.18(\mathrm{dd}, J=8.4,8.4 \mathrm{~Hz}, 1 \mathrm{H}), 4.12-4.06(\mathrm{~m}, 1 \mathrm{H}), 4.01(\mathrm{dd}, J=8.5,6.9 \mathrm{~Hz}, 1 \mathrm{H}), 3.77-3.75(\mathrm{~m}$, $2 \mathrm{H}), 3.74(\mathrm{dd}, J=10.8,4.6 \mathrm{~Hz}, 1 \mathrm{H}), 3.66(\mathrm{dd}, J=10.8,4.3 \mathrm{~Hz}, 1 \mathrm{H}), 3.58(\mathrm{dddd}, J=11.5,4.4$, 4.4, $2.8 \mathrm{~Hz}, 1 \mathrm{H}), 3.35$ (dd, $J=13.4,4.2 \mathrm{~Hz}, 1 \mathrm{H}), 3.28(\mathrm{dd}, J=10.9,10.9 \mathrm{~Hz}, 1 \mathrm{H}), 2.85$ (dd, $J=$ $10.45,3.76 \mathrm{~Hz}, 1 \mathrm{H}), 2.74(\mathrm{dd}, J=13.37,9.77 \mathrm{~Hz}, 1 \mathrm{H}), 1.85-1.77$ (m, apparent br q, $J=$ ca. 10 $\mathrm{Hz}, 1 \mathrm{H}), 1.74-1.70(\mathrm{~m}$, apparent br d, $J=$ ca. $13 \mathrm{~Hz}, 1 \mathrm{H}), 1.68-1.61(\mathrm{~m}, 1 \mathrm{H}), 1.63$ (ddd, $J=12.7$, 2.8, $2.8 \mathrm{~Hz}, 1 \mathrm{H}), 1.50-1.42(\mathrm{~m}, 3 \mathrm{H}), 1.10(\mathrm{ddd}$, apparent q, $J=$ ca. $12 \mathrm{~Hz}, 1 \mathrm{H}), 0.90(\mathrm{~s}, 9 \mathrm{H}), 0.08$ $(\mathrm{s}, 3 \mathrm{H}), 0.07(\mathrm{~s}, 3 \mathrm{H}) . ;{ }^{13} \mathrm{C} \mathrm{NMR}\left(75 \mathrm{MHz} \mathrm{CDCl}_{3}\right) \delta$ 155.5, 139.0, 138.9, 136.6, 129.2, 129.0, 128.57, 128.56, 127.73, 127.66, 127.6, 127.5, 127.0, 76.8, 74.6, 74.4, 71.0, 70.8, 67.2, 65.8, 61.1, 60.1, 59.1, 39.2, 36.0, 35.7, 33.8, 31.7, 29.9, 29.3, 26.2, 18.7, -5.2 (2C); MS (ESI) $\mathrm{m} / z$ (relative intensity) $671.33\left([\mathrm{M}+1]^{+} 100 \%\right)$; HRMS (ESI) $\mathrm{m} / z$ calcd. for $\mathrm{C}_{40} \mathrm{H}_{54} \mathrm{~N}_{2} \mathrm{O}_{5} \mathrm{NaSi} 693.3700$ $\left([\mathrm{M}+\mathrm{Na}]^{+}\right)$; found 693.3712 .

1. Walborsky, H. M.; Barash L.; Davis, T. C. Tetrahedron 1963, 19, 2333-2351.

2. Prepared by reduction of the diacid: Applequist, D. E.; Werner, N. D. J. Org. Chem. 1963, $28,48-54$.

3. Glossop, P.; Jones, D. W. J. Chem. Soc. Perkin Trans. 1 1996, 501-506.

4. Rearrangement and loss of $\mathrm{CH}_{2}=\mathrm{CHOH}$ gives $[\mathrm{M}-44]^{+}$, diagnostic for $\mathrm{RCH}_{2} \mathrm{CHO}$. Silverstein, R. M.; Bassler, G. C.; Morrill, T. C. Spectrometric Identification of Organic Compounds, 4th edition; Wiley: New York, 1981, p. 26.

5. Friestad, G. K.; Qin, J. J. Am. Chem. Soc. 2001, 123, 9922-9923. 


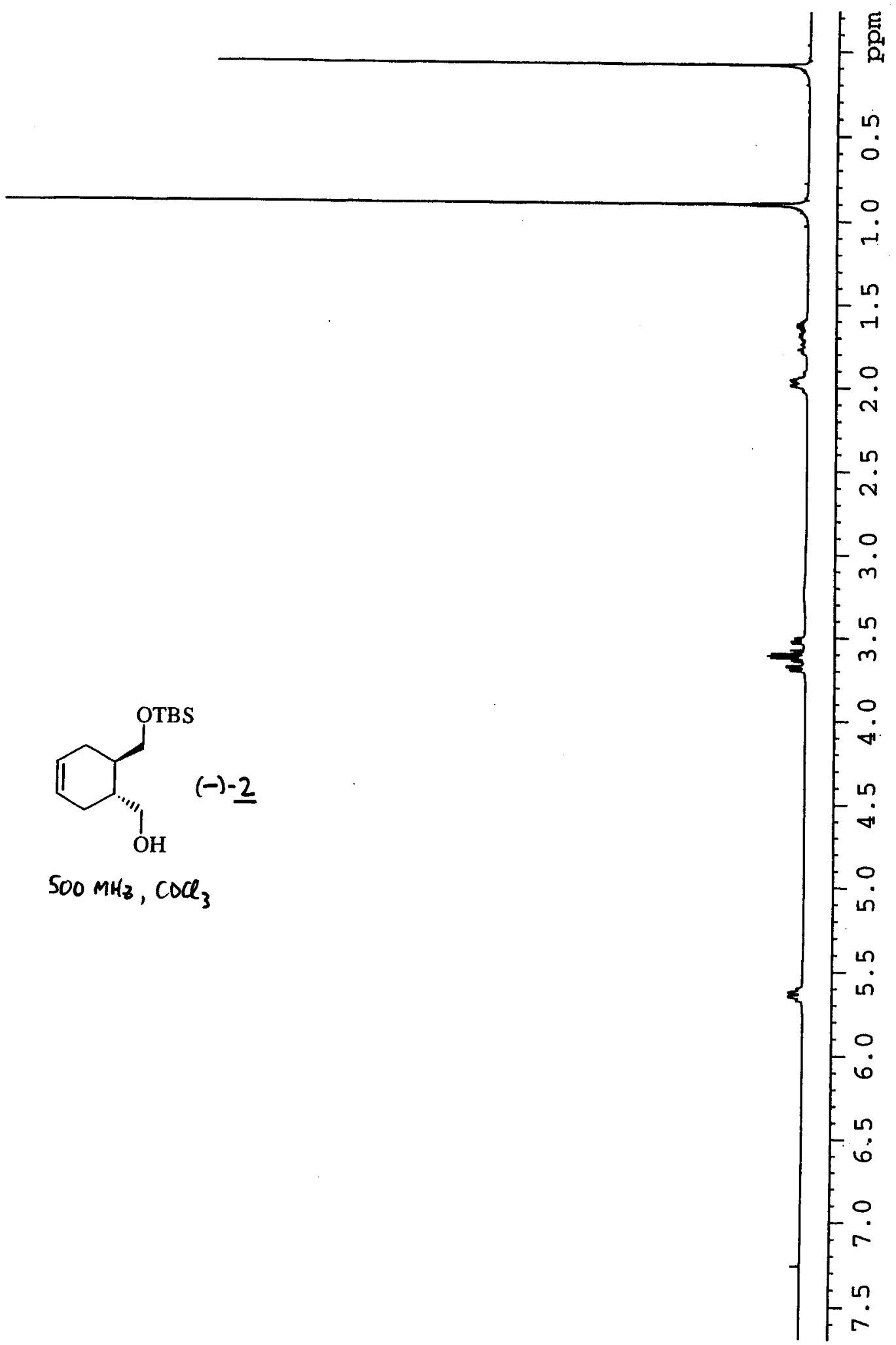




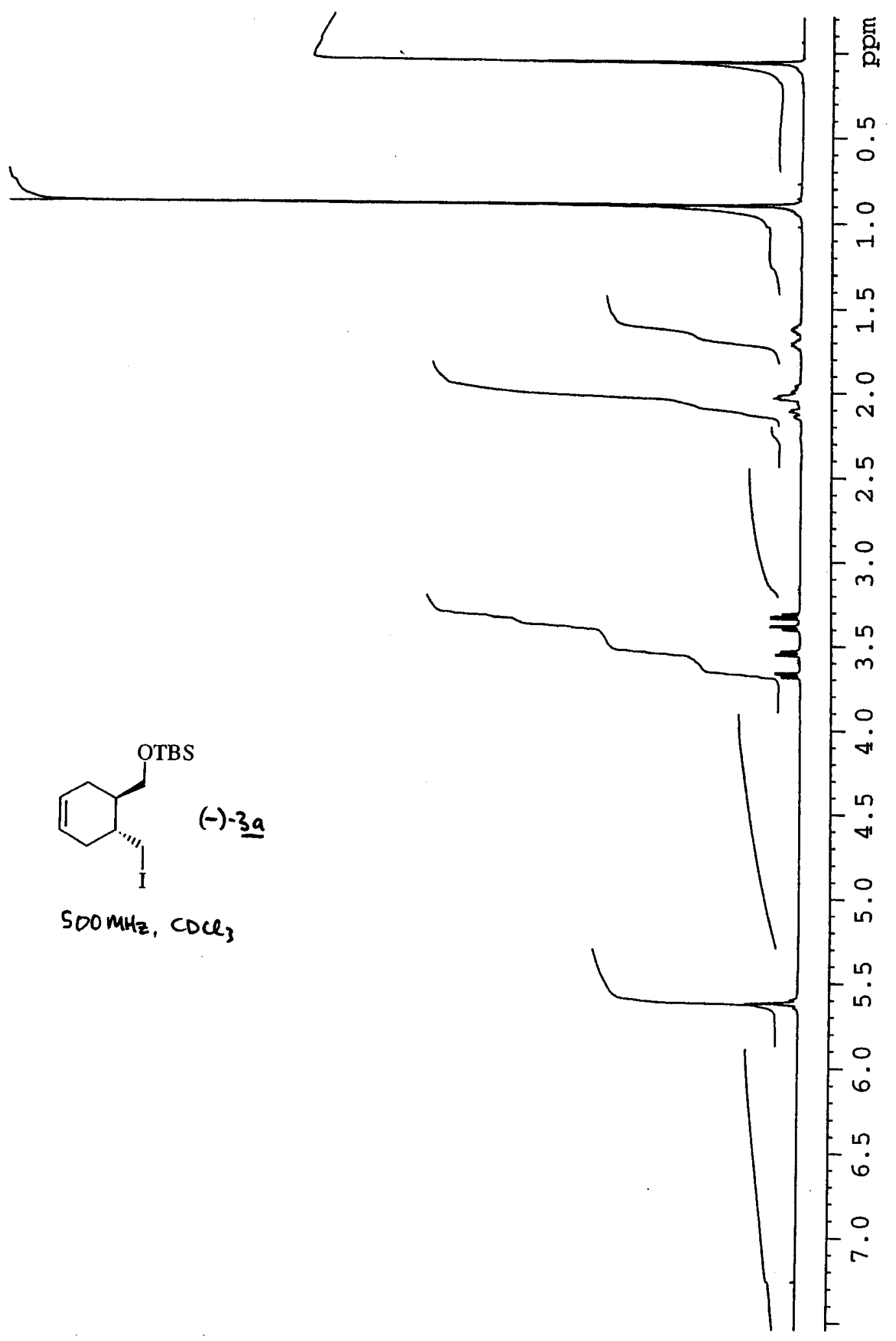




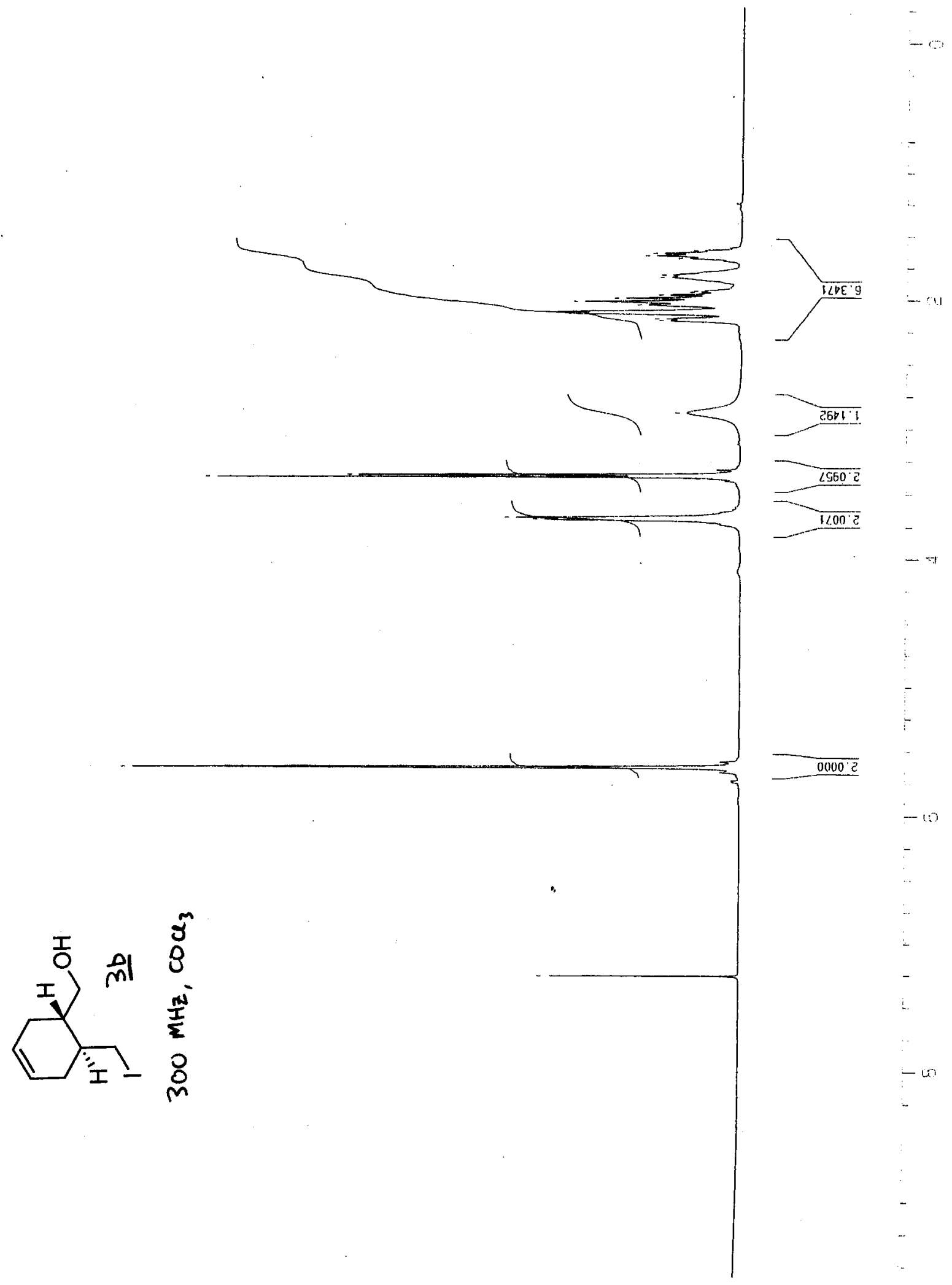




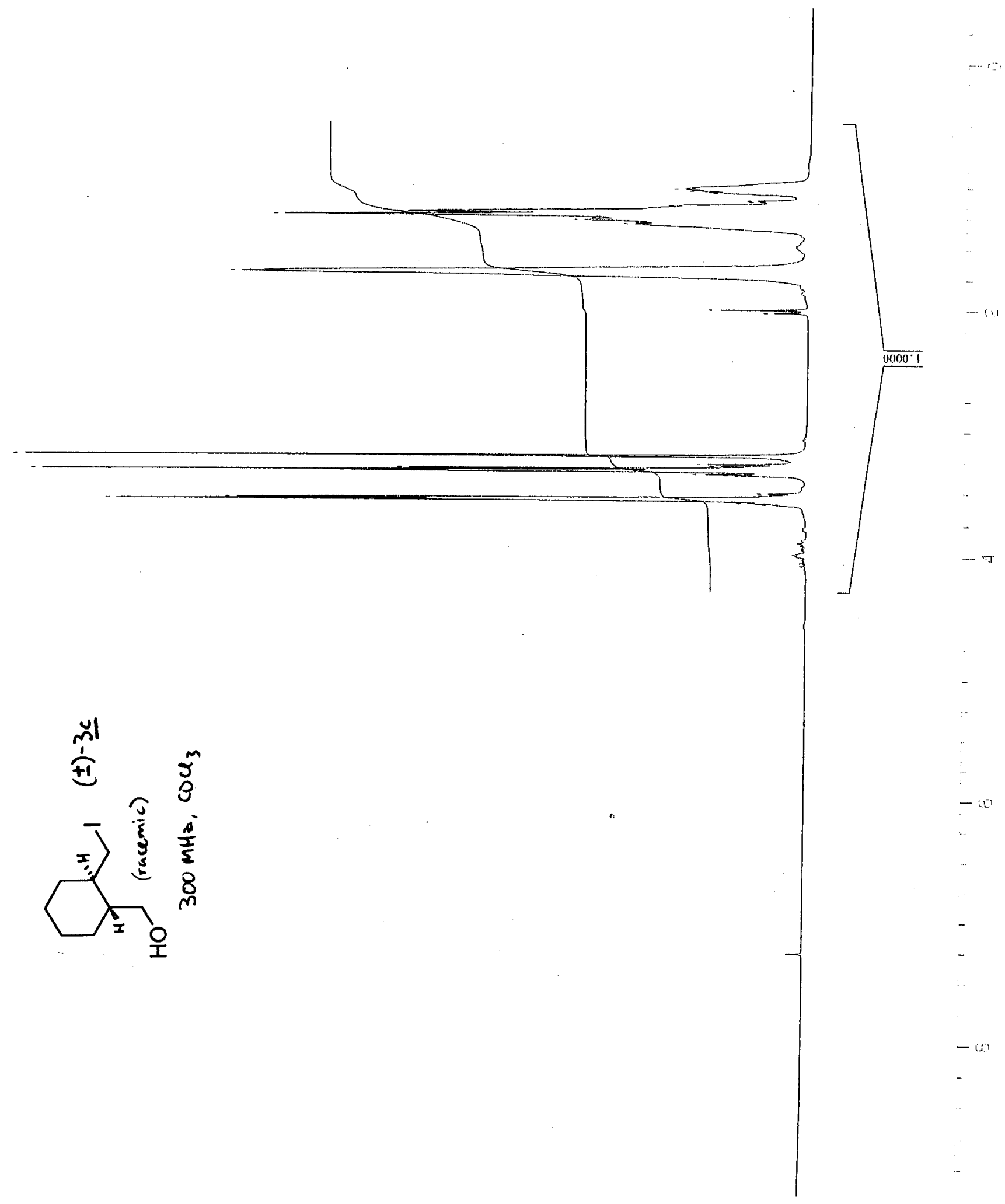




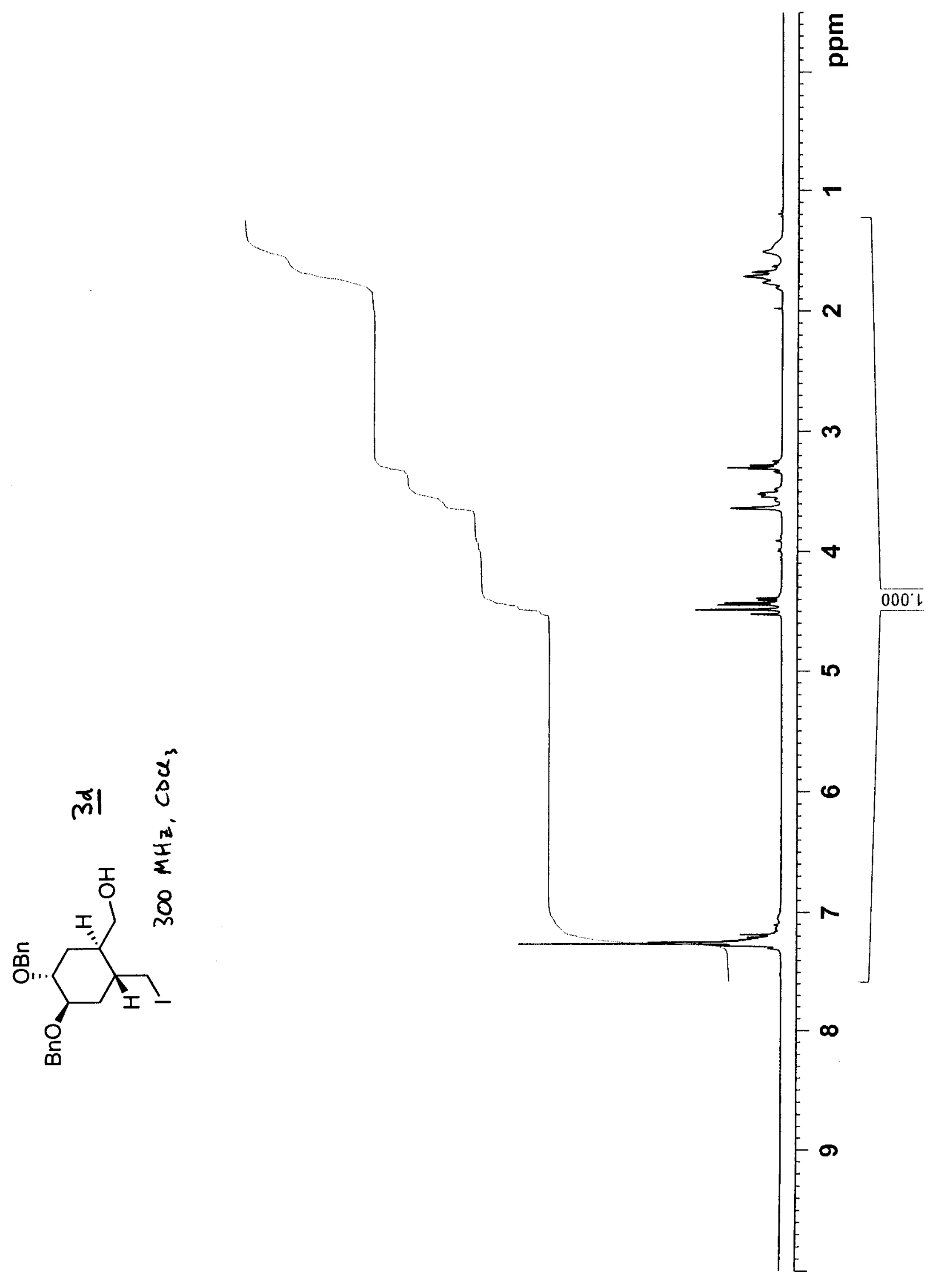




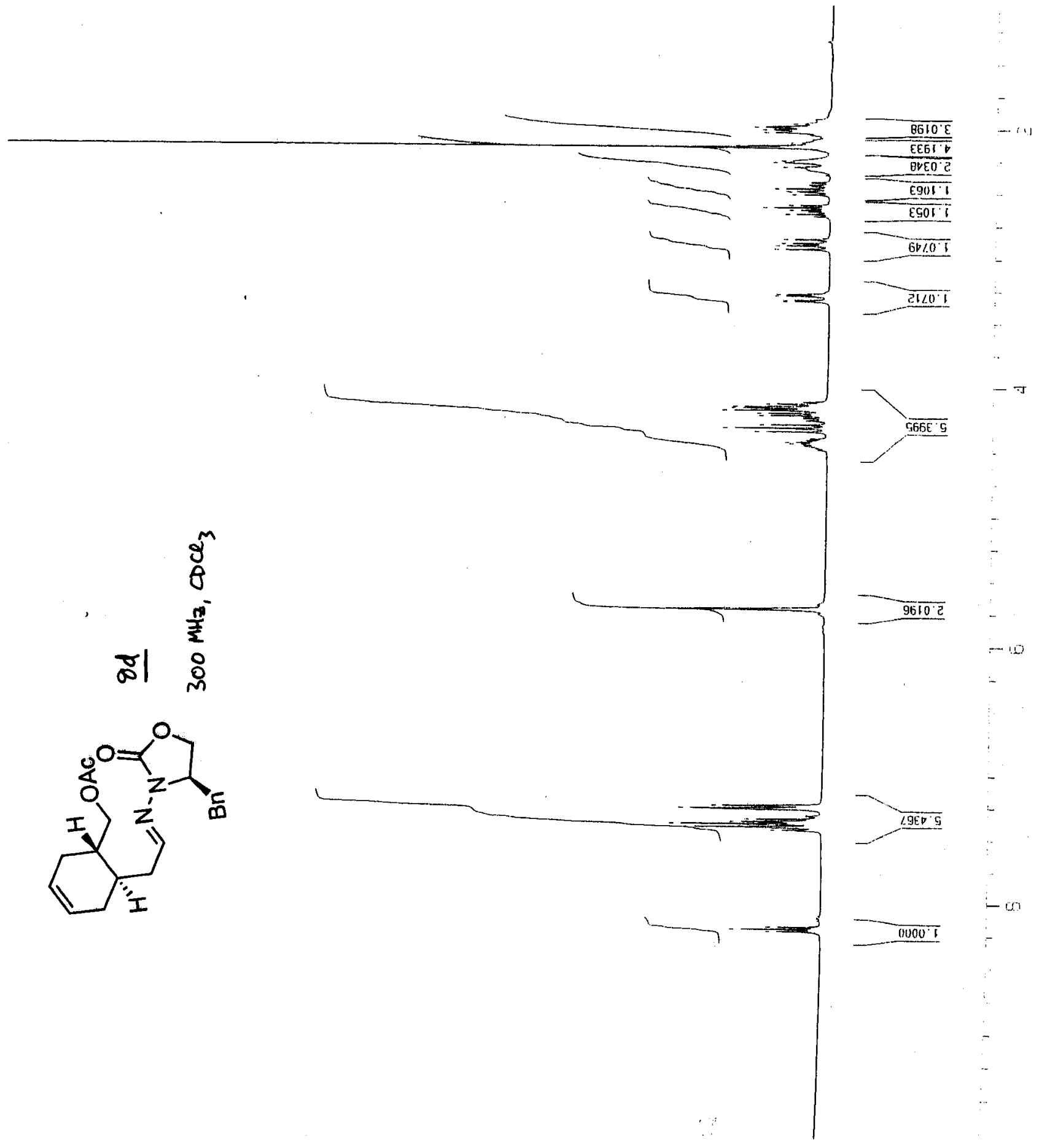




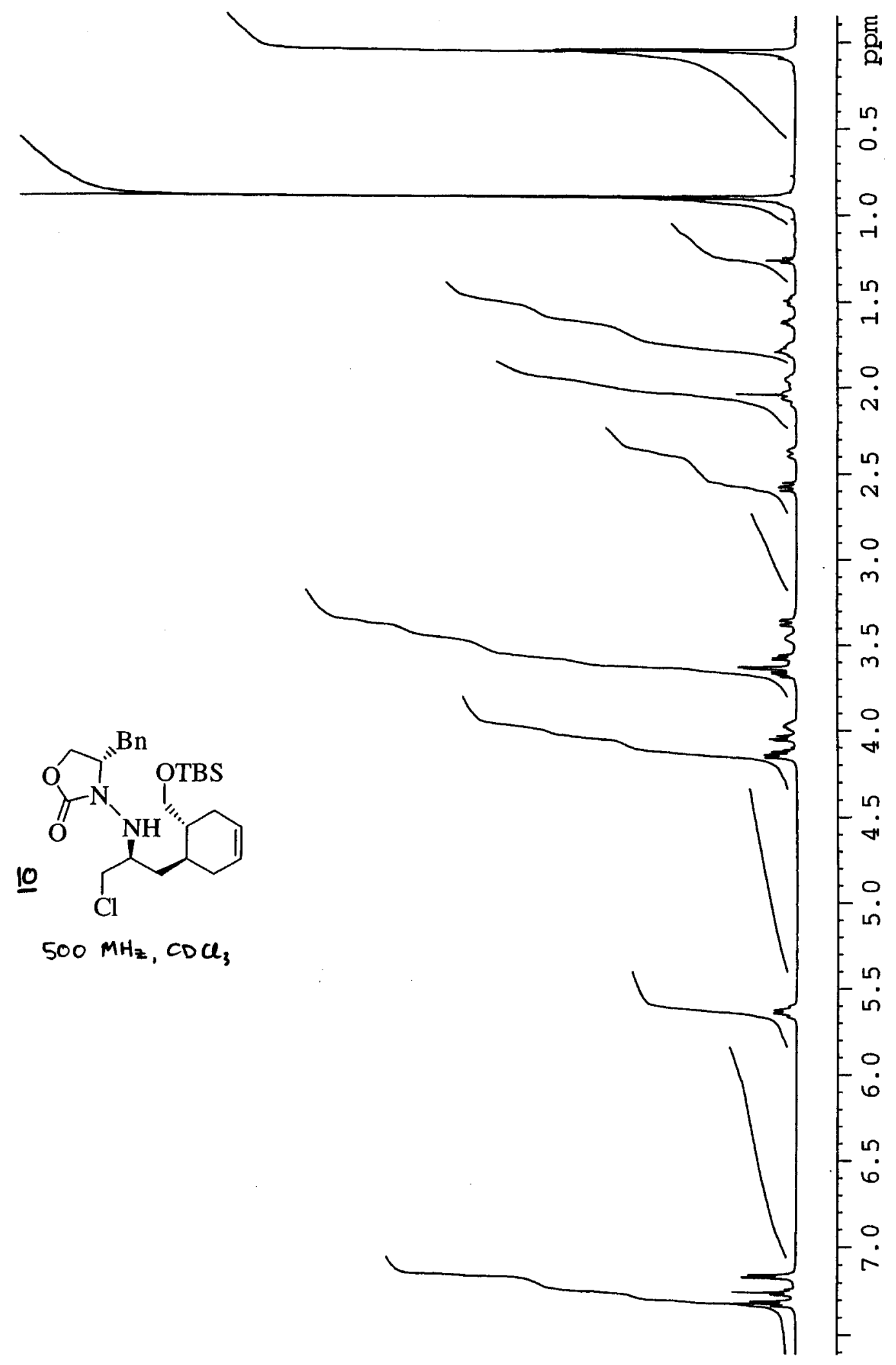




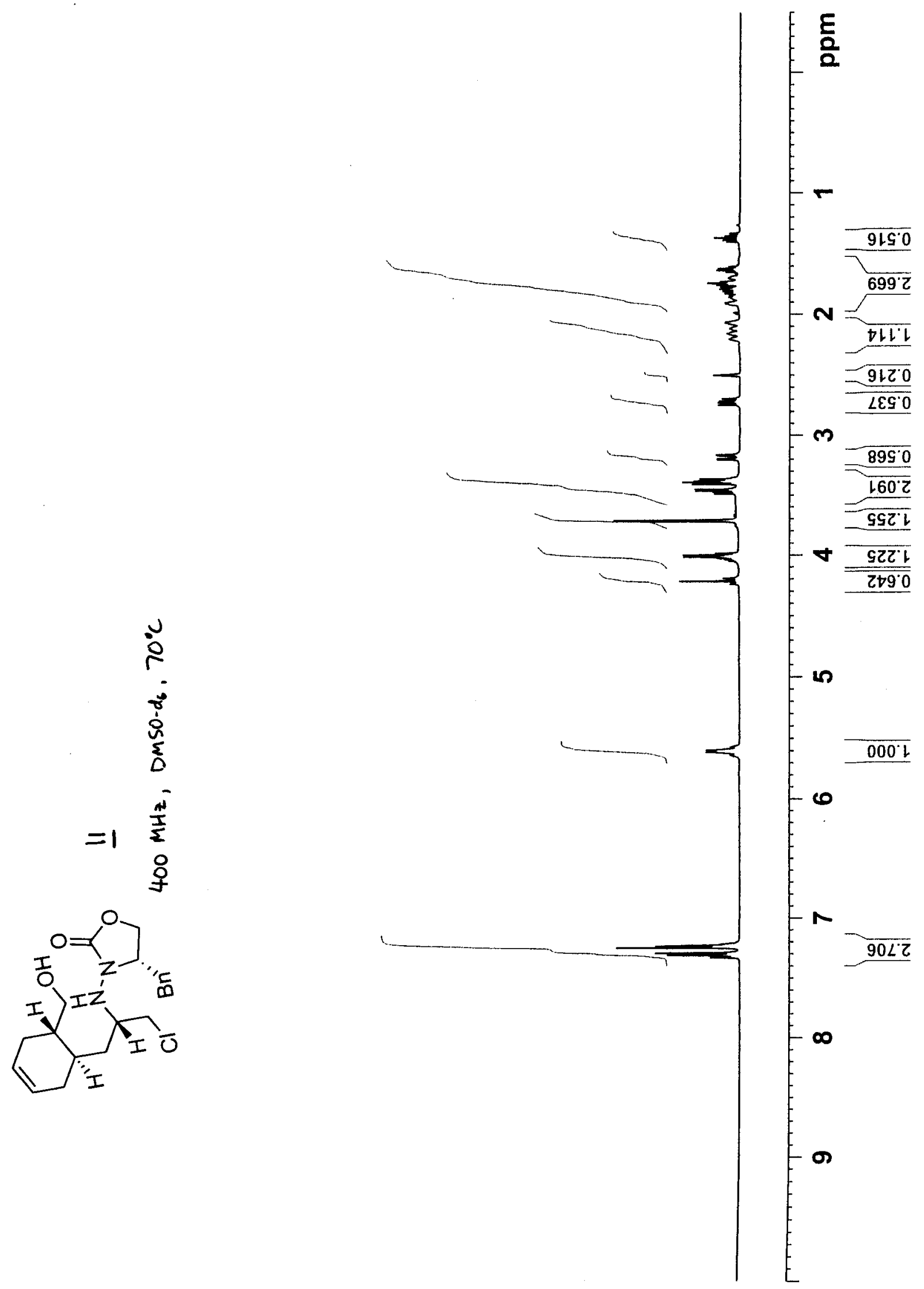




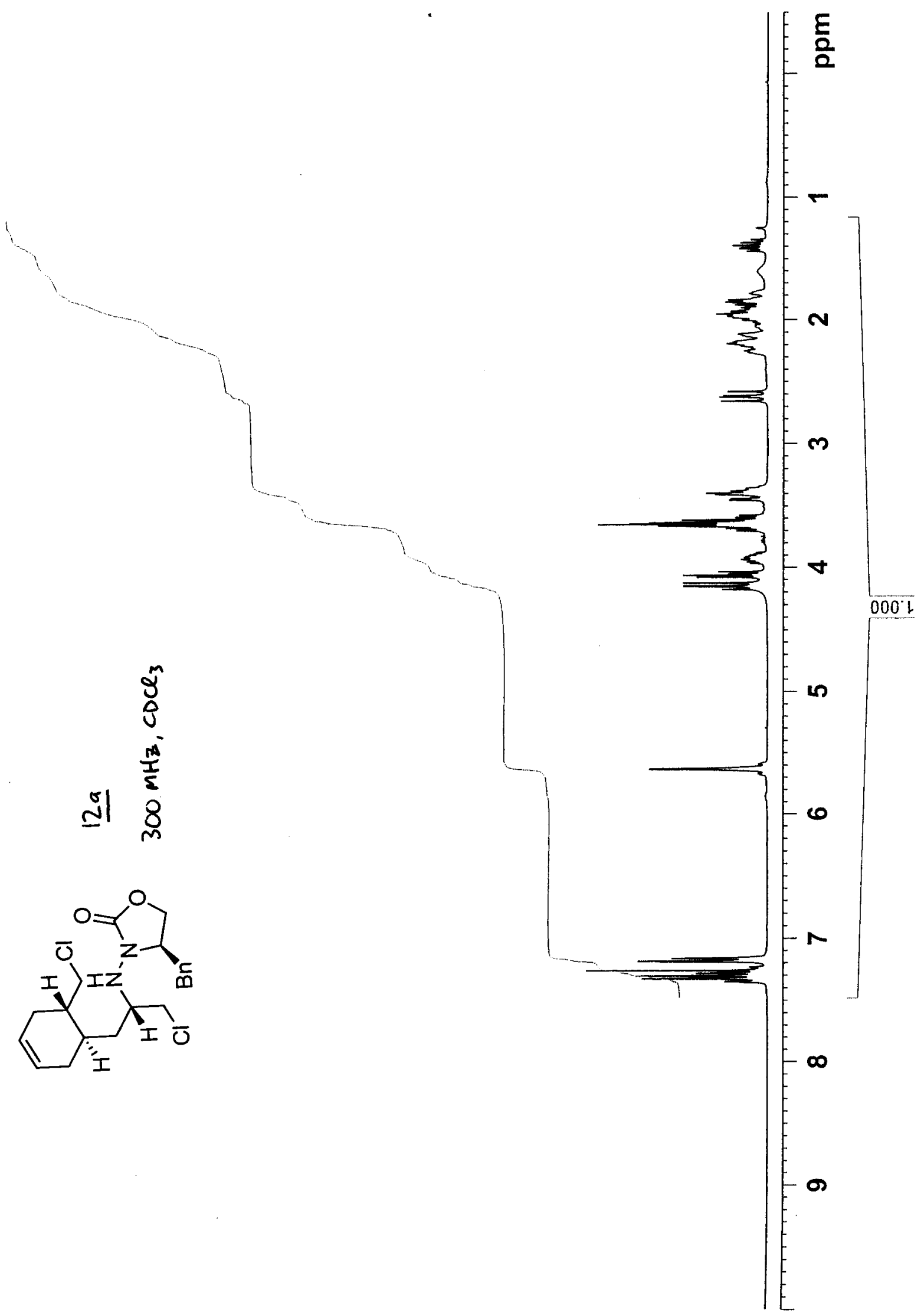




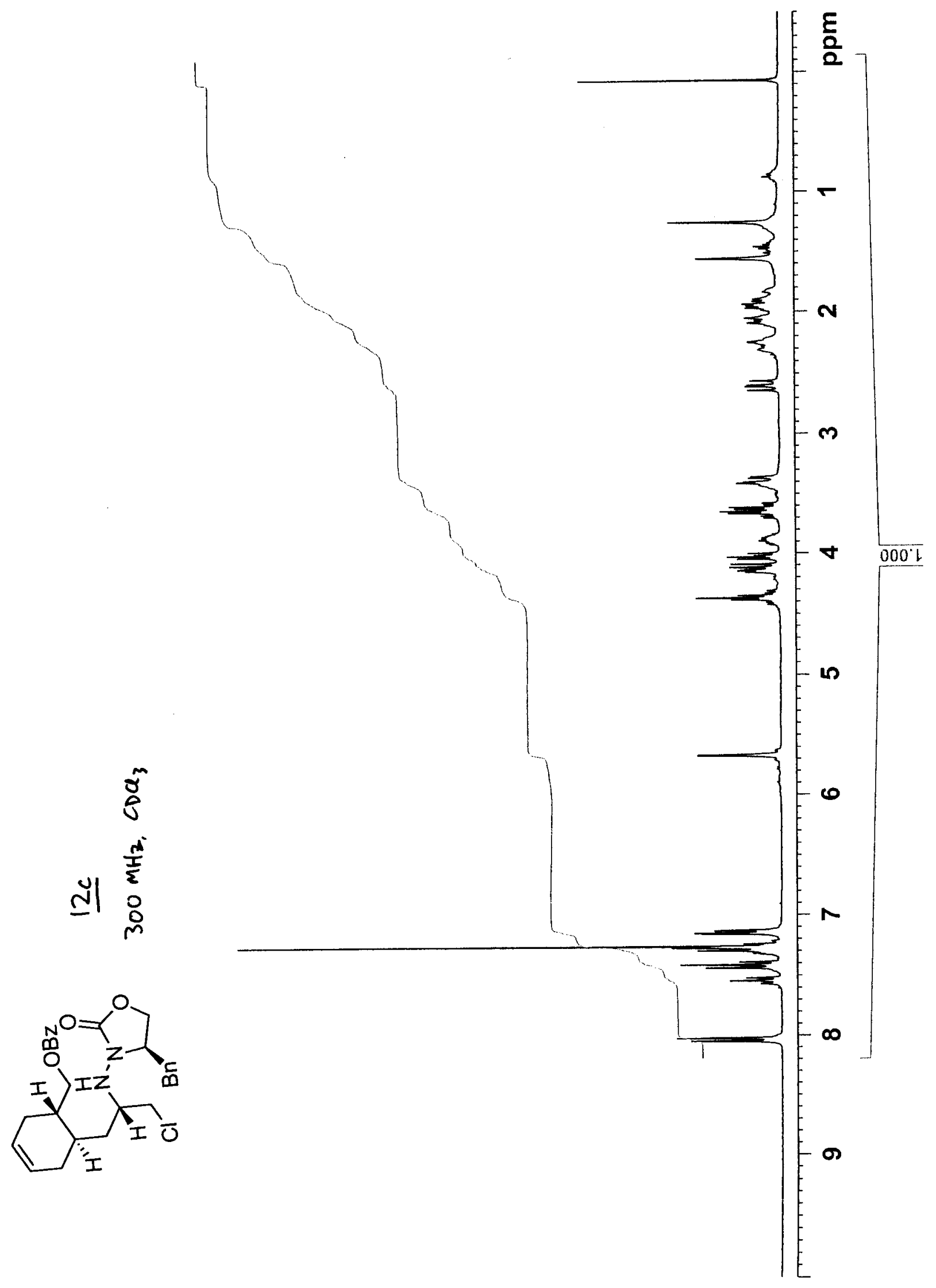




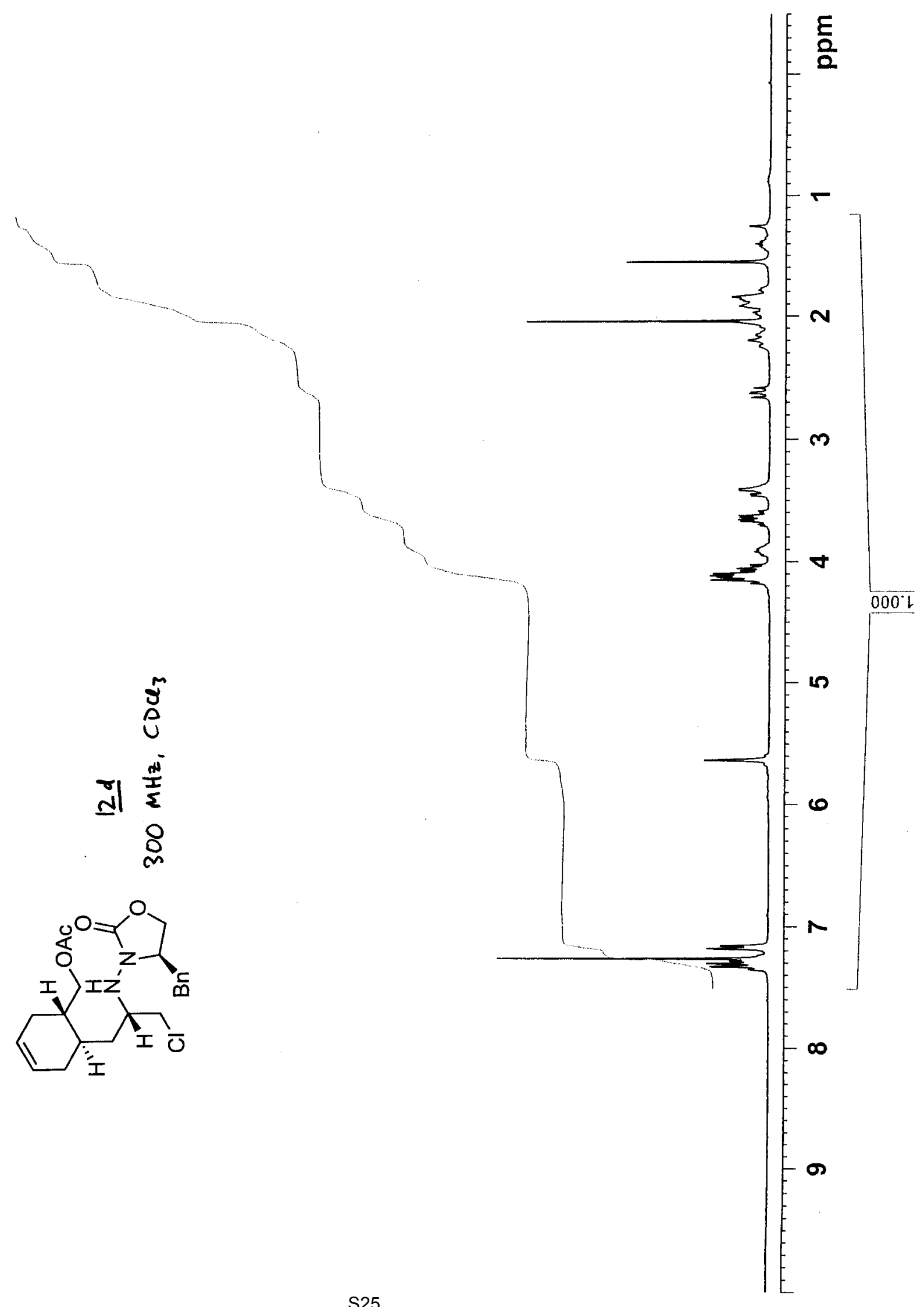




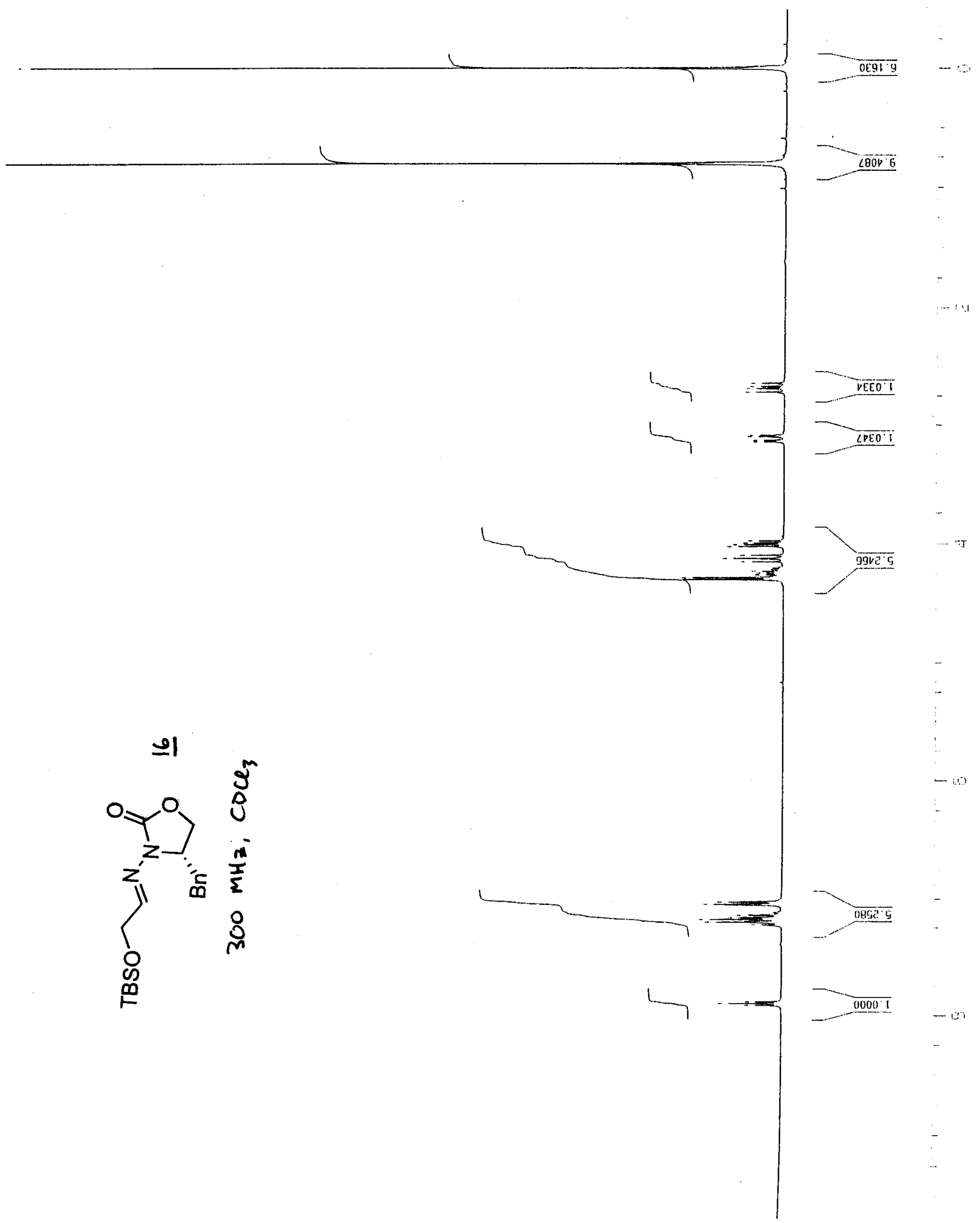




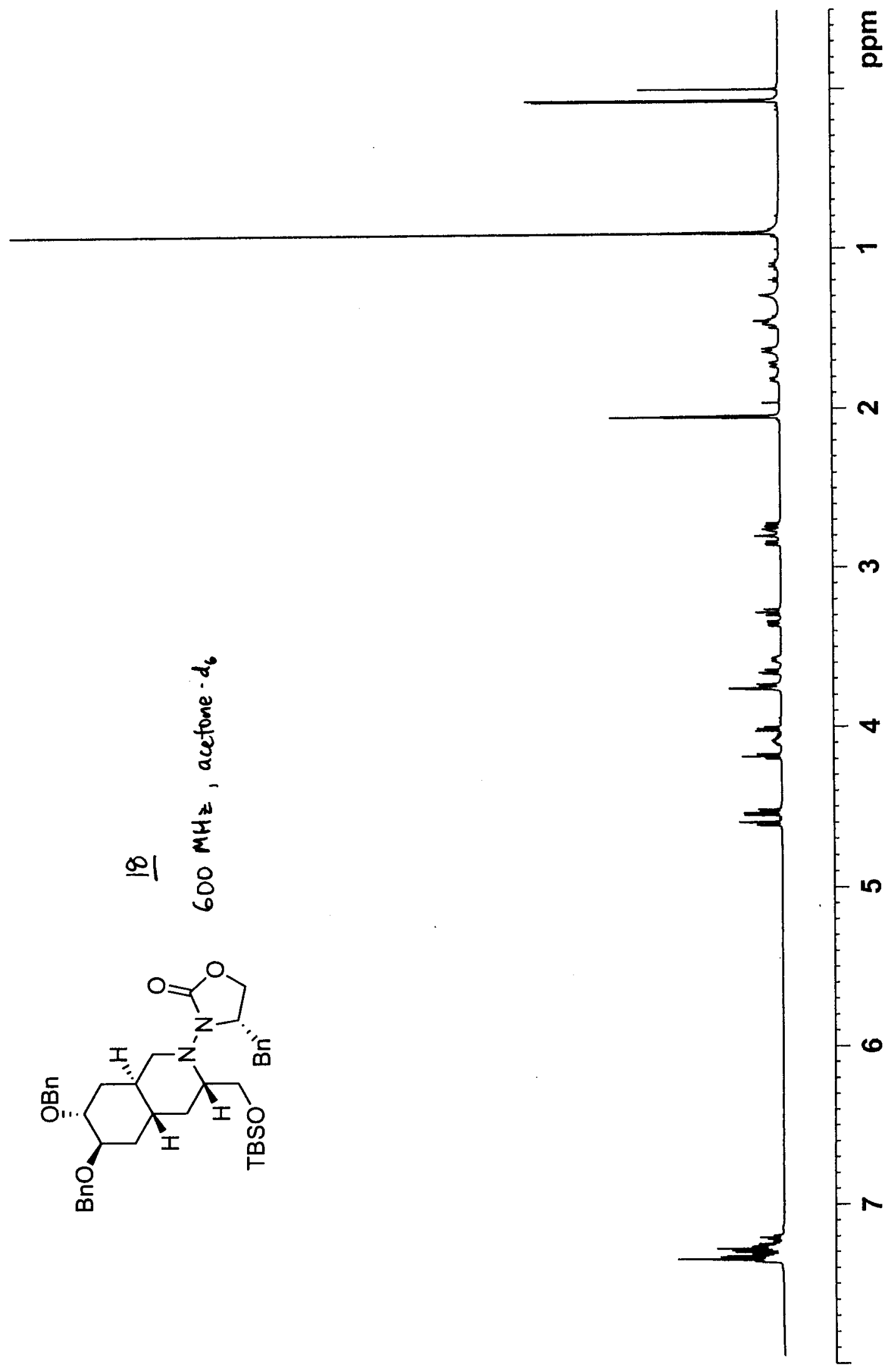




\section{Peak Assignments and NOE Data for Compound 18}

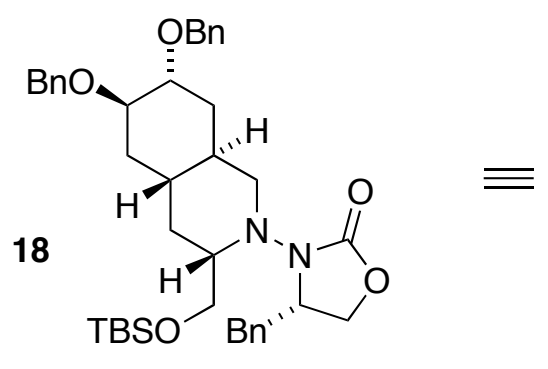

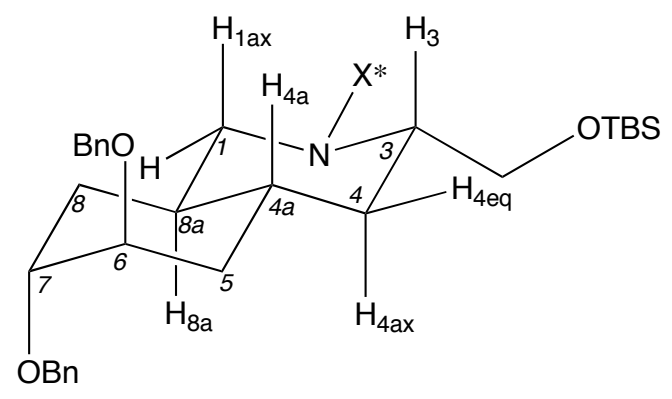

\begin{tabular}{|c|c|c|c|}
\hline \multicolumn{2}{|c|}{ Peak Assignments by Hydrogen Number } & \multicolumn{2}{|c|}{ Peak Assignments by Chemical Shift } \\
\hline Hydrogen & Chemical Shift & Chemical Shift & Hydrogen \\
\hline $\mathrm{H}_{1 \mathrm{ax}}$ & 3.28 & 1.10 & $\mathrm{H}_{4 \mathrm{ax}}$ \\
\hline $\mathrm{H}_{1 \mathrm{eq}}$ & 2.85 & $1.50-1.42$ & $\mathrm{H}_{4 \mathrm{a}}, \mathrm{H}_{5 \mathrm{eq}}, \mathrm{H}_{8 \mathrm{ax}}$ \\
\hline $\mathrm{H}_{3}$ & 3.58 & 1.63 & $\mathrm{H}_{4 \mathrm{eq}}$ \\
\hline $\mathrm{H}_{4 \mathrm{ax}}$ & 1.10 & $1.68-1.61$ & $\mathrm{H}_{8 \mathrm{a}}$ \\
\hline $\mathrm{H}_{4 \mathrm{eq}}$ & 1.63 & $1.74-1.70$ & $\mathrm{H}_{8 \mathrm{eq}}$ \\
\hline $\mathrm{H}_{4 \mathrm{a}}$ & $1.50-1.42$ & $1.85-1.79$ & $\mathrm{H}_{5 \mathrm{ax}}$ \\
\hline $\mathrm{H}_{5 \mathrm{ax}}$ & $1.85-1.79$ & 2.74 & $X *$ (oxazolidinone) \\
\hline $\mathrm{H}_{5 \mathrm{eq}}$ & $1.50-1.42$ & 2.85 & $\mathrm{H}_{1 \mathrm{eq}}$ \\
\hline $\mathrm{H}_{6}$ & $3.77-3.75$ & 3.28 & $\mathrm{H}_{1 \mathrm{ax}}$ \\
\hline $\mathrm{H}_{7}$ & $3.77-3.75$ & 3.35 & $X *$ (oxazolidinone) \\
\hline $\mathrm{H}_{8 \mathrm{ax}}$ & $1.50-1.42$ & 3.58 & $\mathrm{H}_{3}$ \\
\hline $\mathrm{H}_{8 \mathrm{eq}}$ & $1.74-1.70$ & 3.66 & $\mathrm{CH}_{2} \mathrm{OTBS}$ \\
\hline $\mathrm{H}_{8 \mathrm{a}}$ & $1.68-1.61$ & 3.74 & $\mathrm{CH}_{2} \mathrm{OTBS}$ \\
\hline $\mathrm{CH}_{2} \mathrm{OTBS}$ & $3.66,3.74$ & $3.77-3.75$ & $\mathrm{H}_{6}, \mathrm{H}_{7}$ \\
\hline $2 \times \mathrm{OBn}$ & $4.61,4.60,4.54,4.53$ & 4.01 & $X^{*}$ (oxazolidinone) \\
\hline \multirow[t]{6}{*}{ X* (oxazolidinone) } & $4.18,4.12-4.06$ & $4.12-4.06$ & $X *$ (oxazolidinone) \\
\hline & $4.01,3.35,2.74$ & 4.18 & X* (oxazolidinone) \\
\hline & & 4.53 & OBn \\
\hline & & 4.54 & $\mathrm{OBn}$ \\
\hline & & 4.60 & OBn \\
\hline & & 4.61 & OBn \\
\hline
\end{tabular}

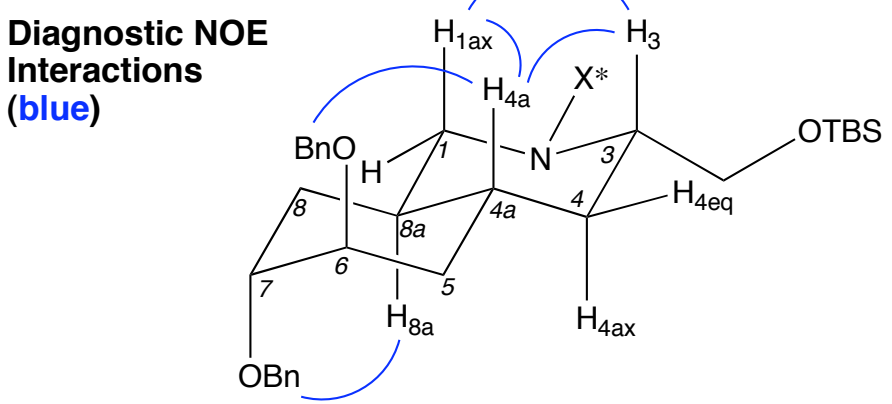


COSY spectrom of $18\left(600 \mathrm{MHz}\right.$, acetone $\left.\cdot d_{6}\right)$
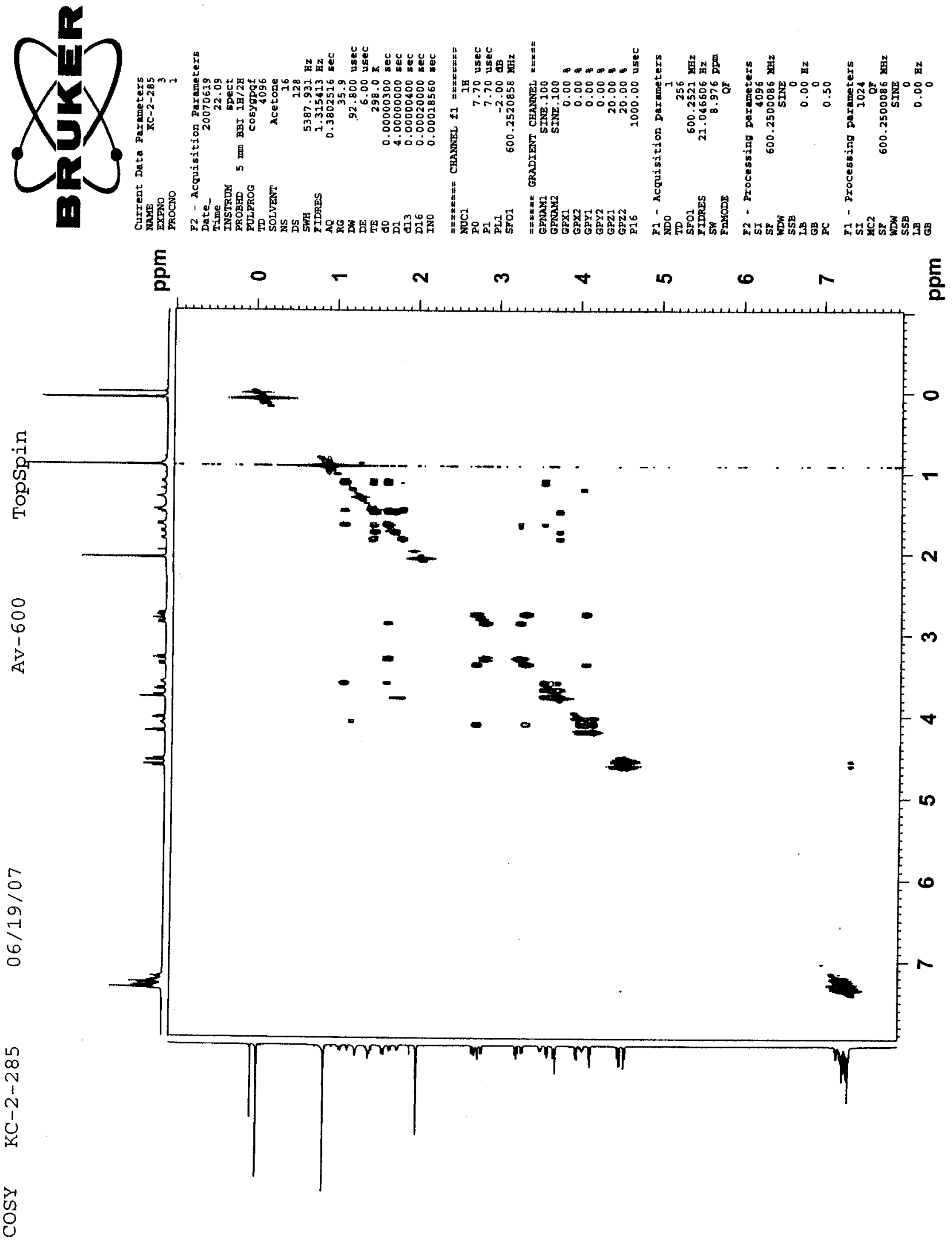
NOESY spectrum of $18\left(600 \mathrm{MHz}\right.$, acetone- $\left.d_{6}\right)$

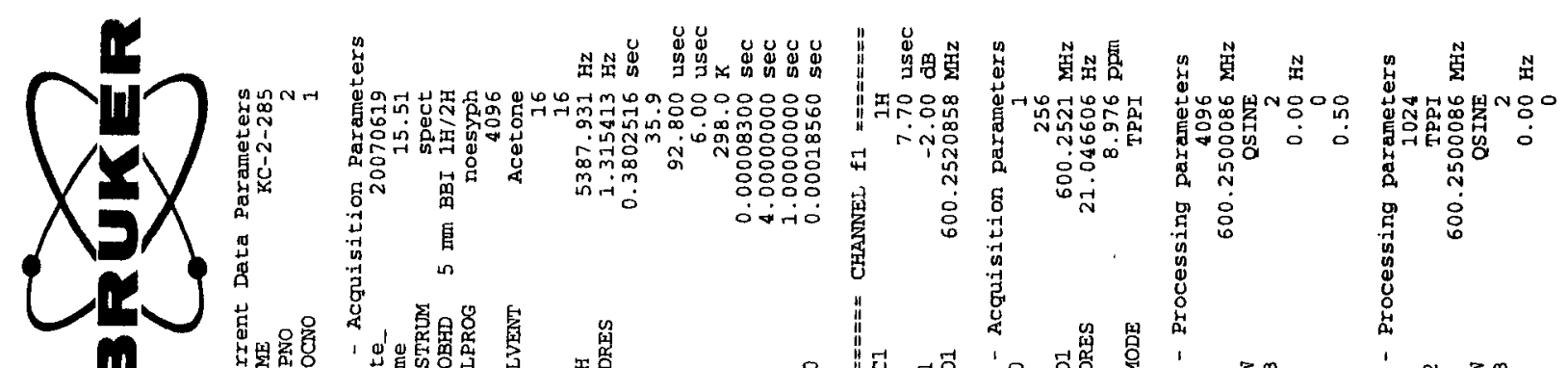

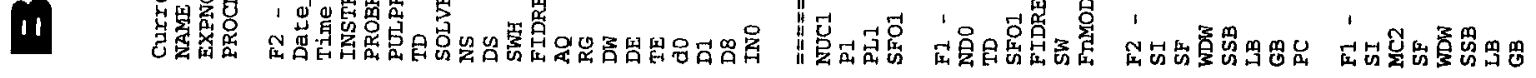

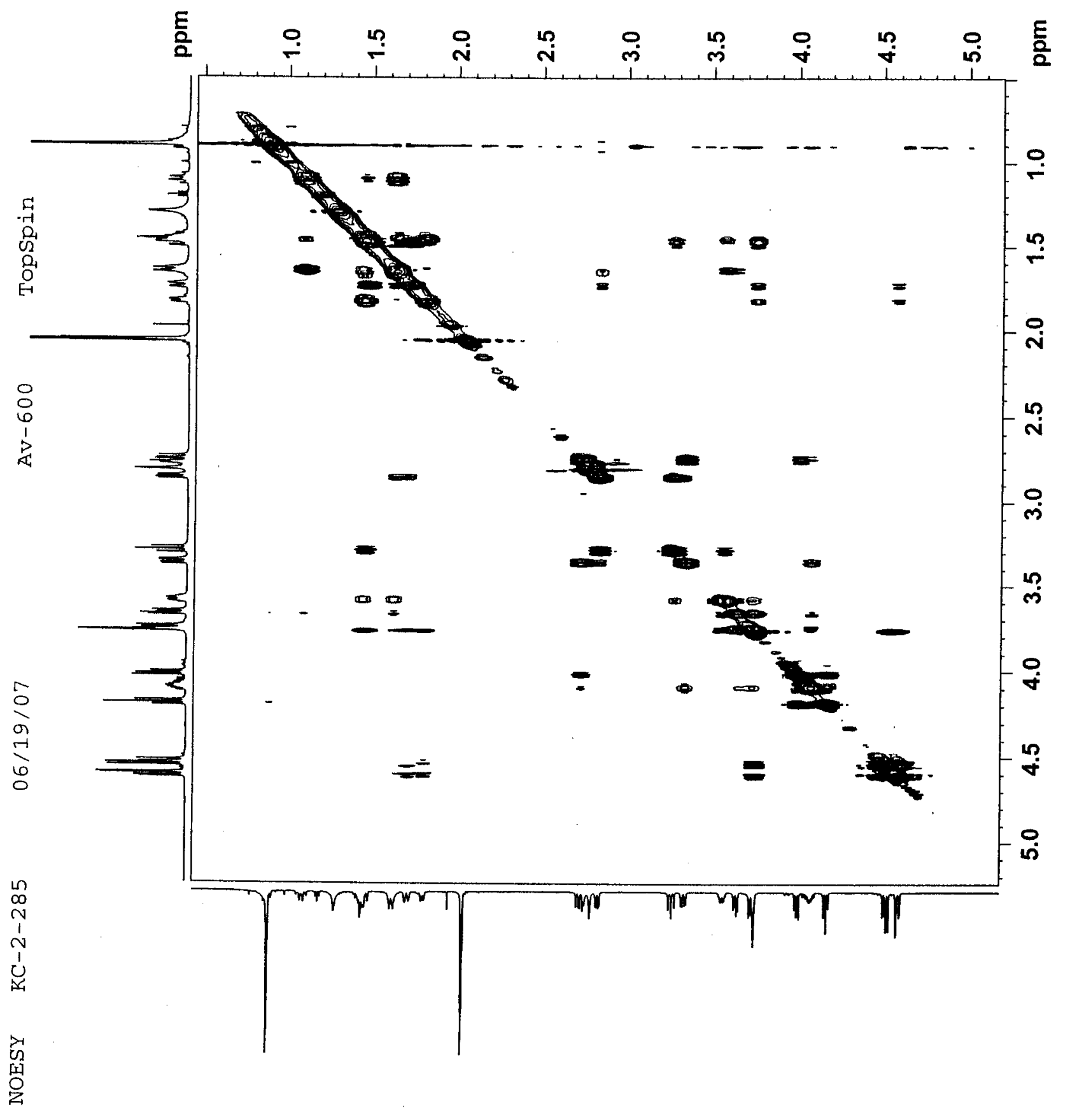




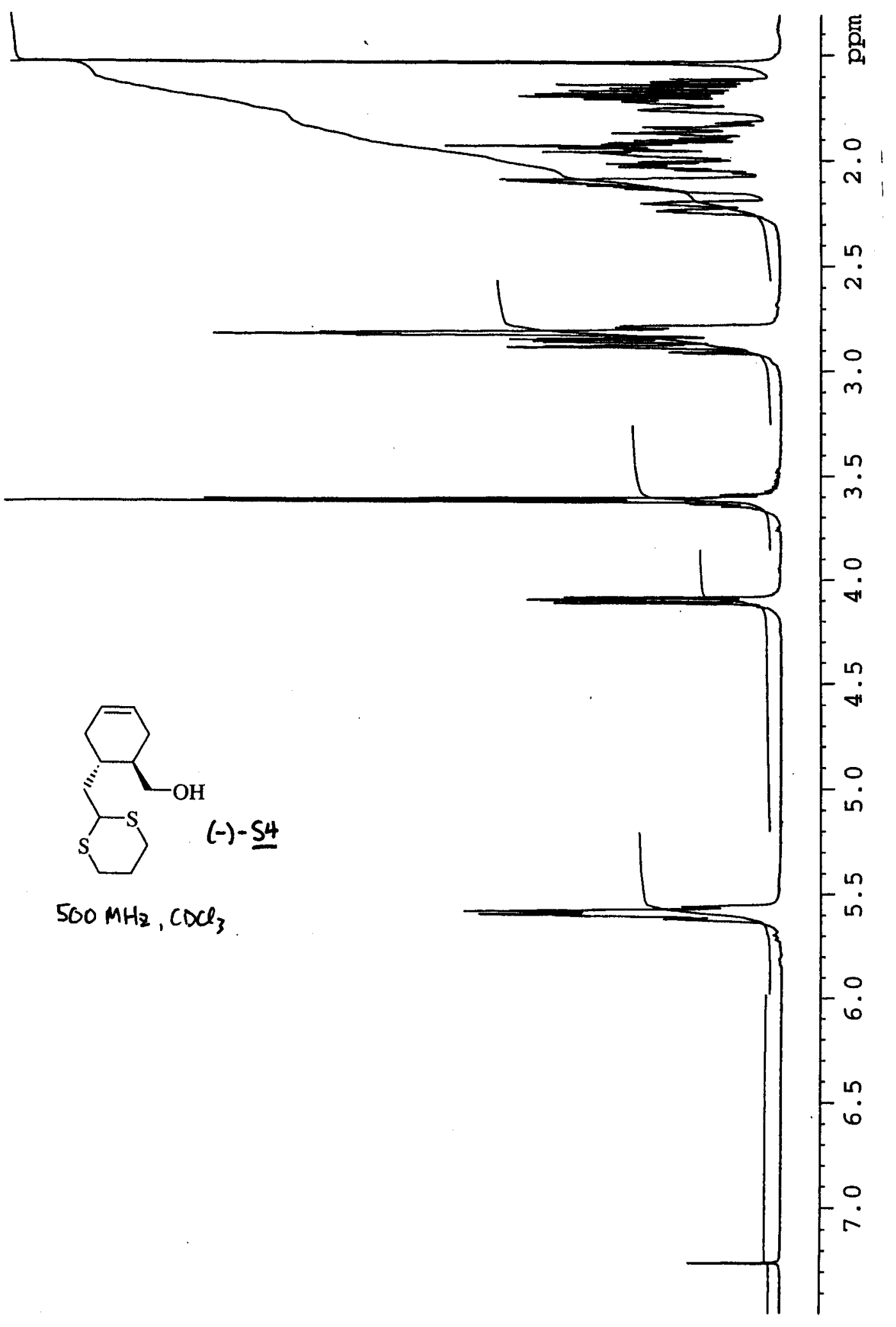




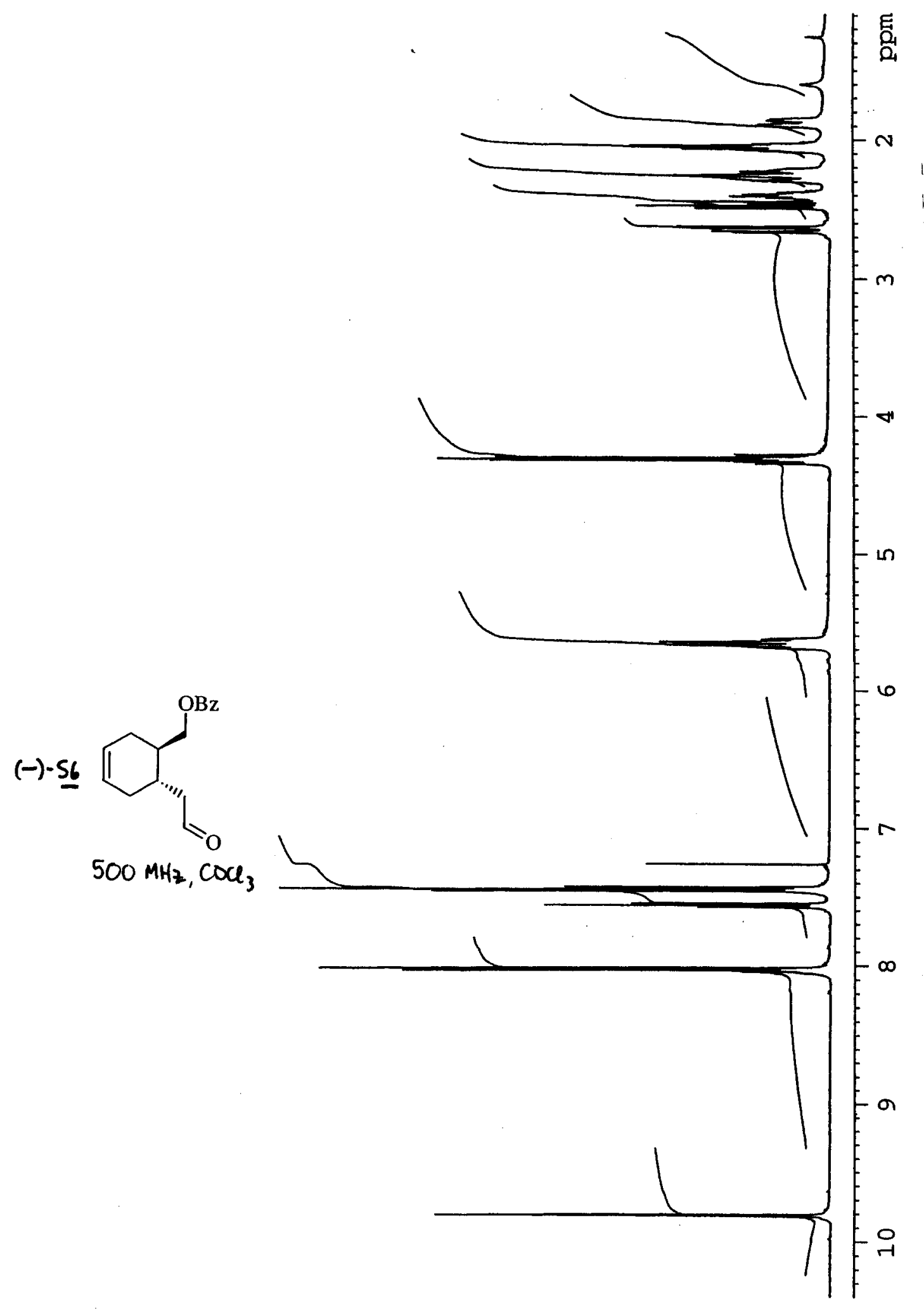




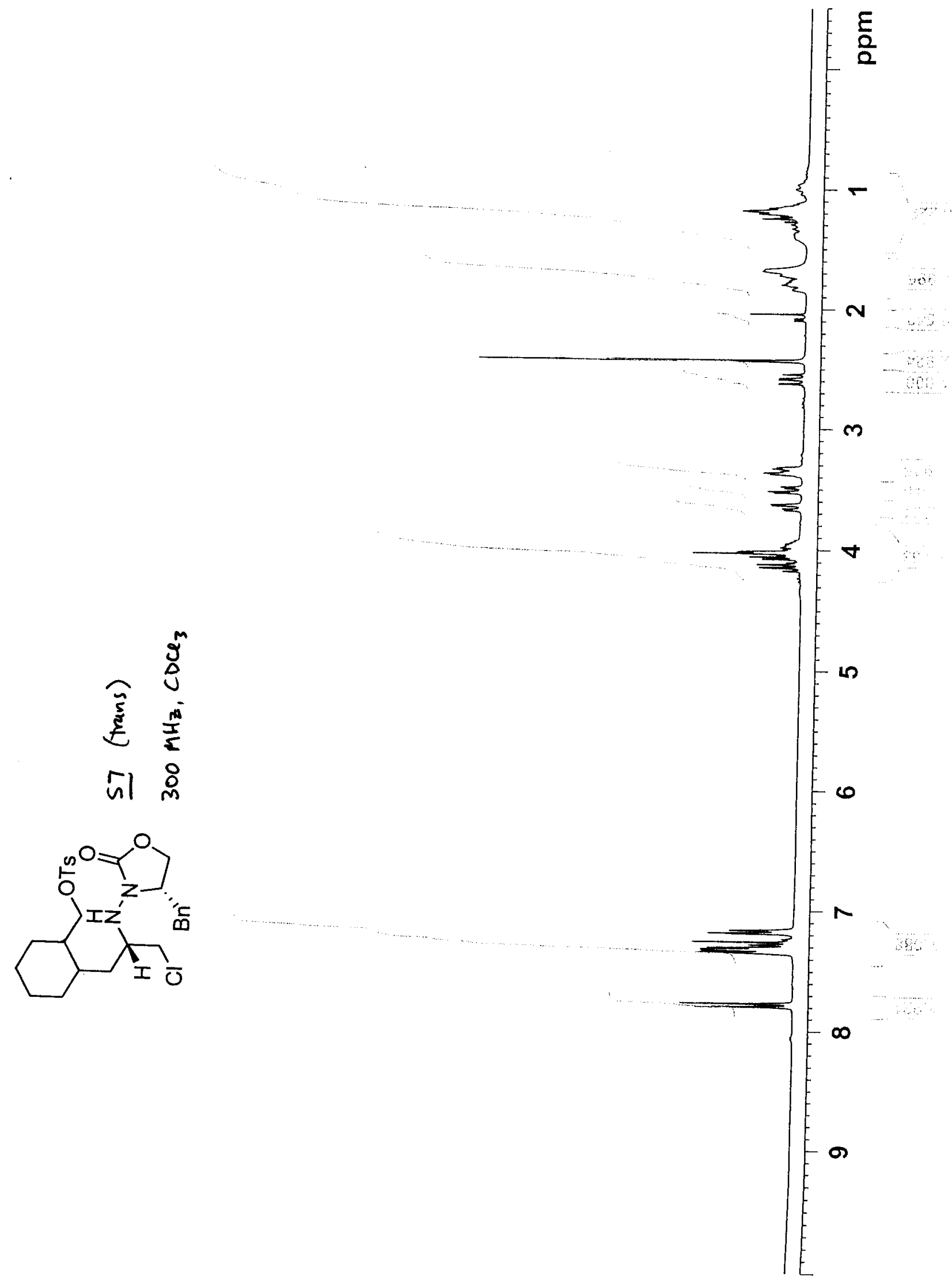




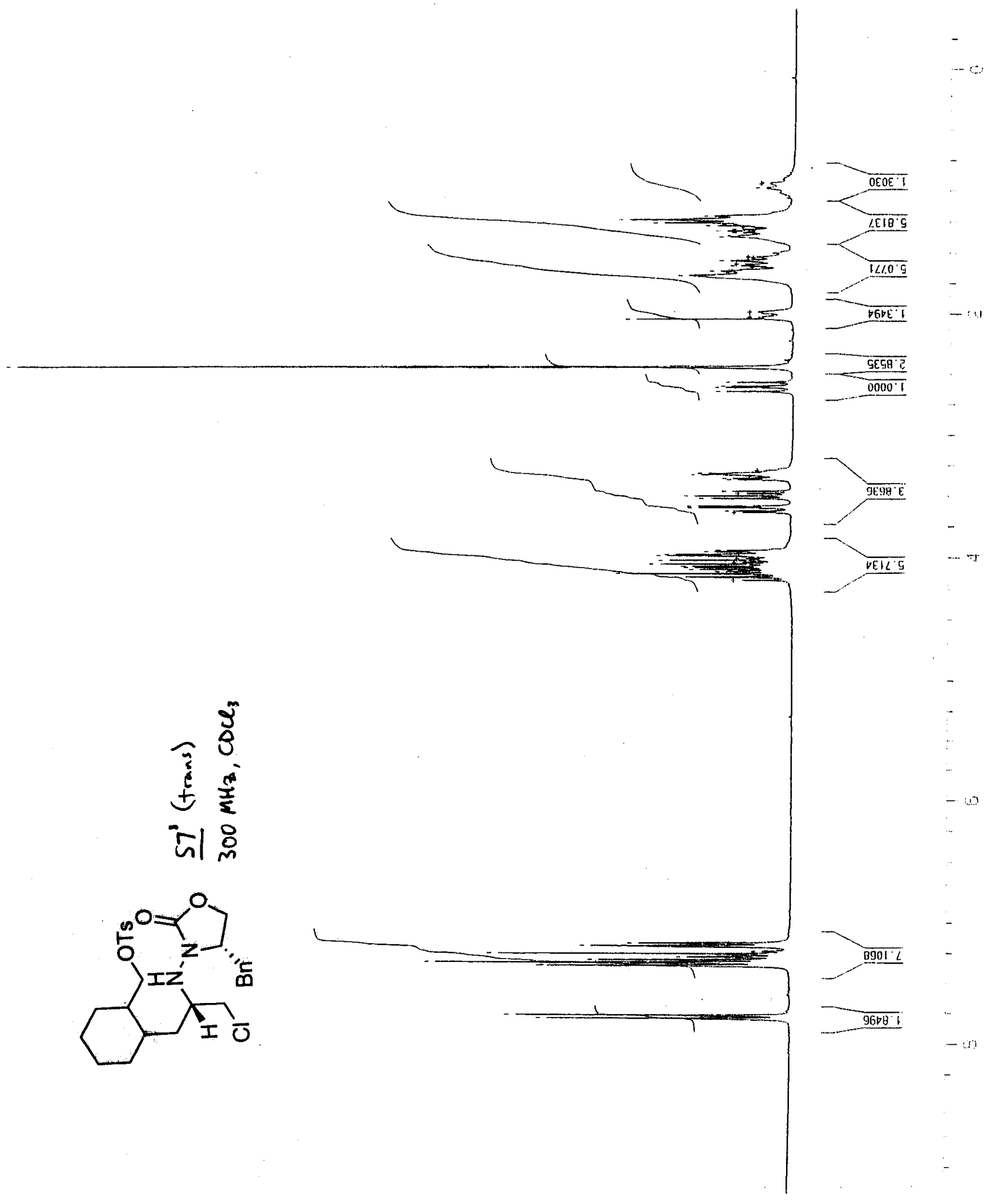




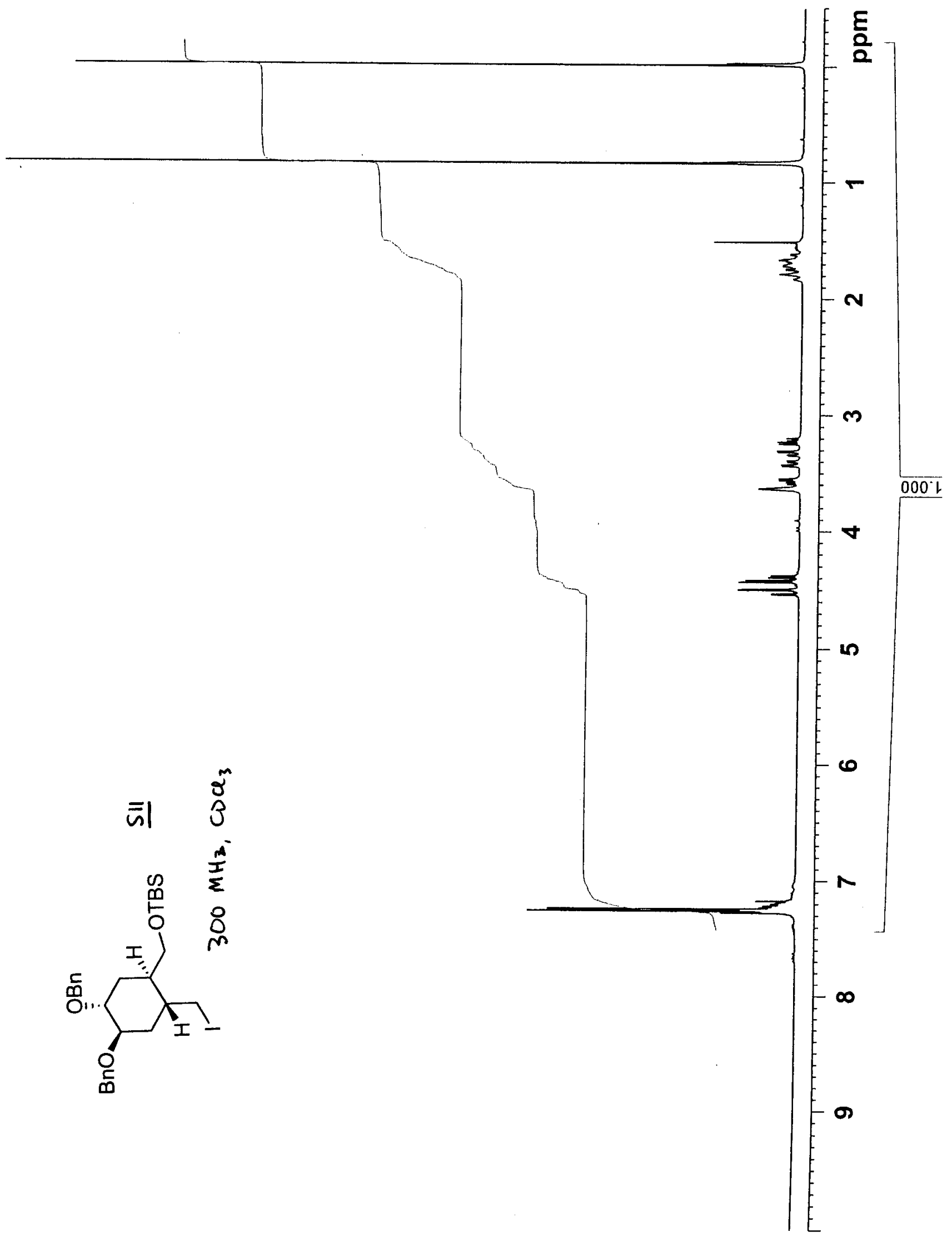


X-Ray Crystallographic Structure of Compound S9

(also named Fri06_1 and fri61 in the following pages)
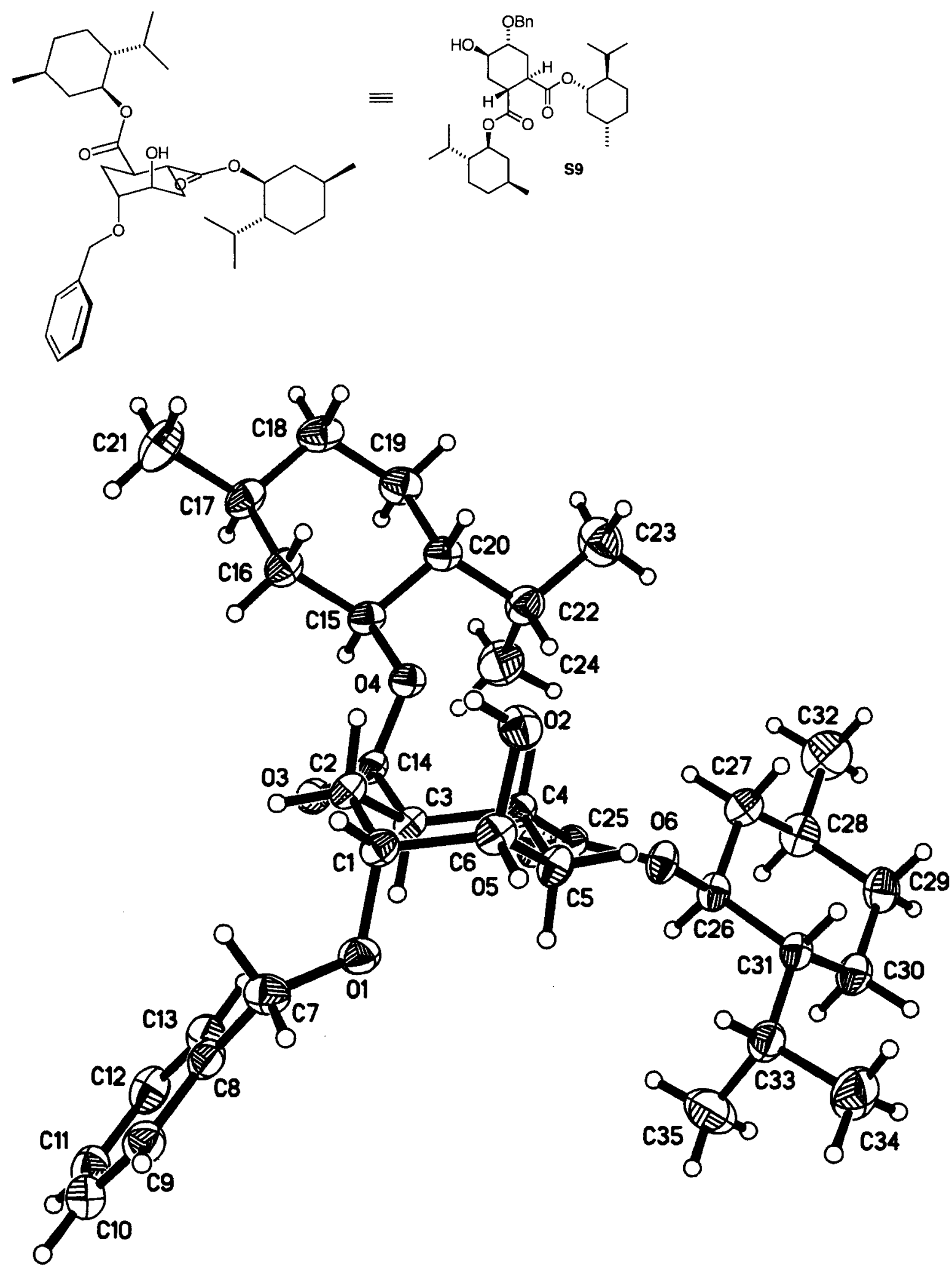
A colorless prism $(0.42 \times 0.20 \times 0.17 \mathrm{~mm})$ was isolated from the sample and mounted with grease on the tip of a glass capillary epoxied to a brass pin and placed on the diffractometer with the long crystal dimension (unit cell a-axis) approximately parallel to the diffractometer phi axis.

Data for Fri06 I were collected with a Nonius KappaCCD diffractometer (Mo K-alpha radiation, graphite monochromator) at 150 (2) K (cold N2 gas stream) using standard CCD techniques yielding 26655 data. Lorentz and polarization corrections were applied. A correction for absorption using the multi-scan technique was applied ( $\max =0.987$, Tmin $=0.969)$. Equivalent data were averaged yielding 4015 unique data ( $\mathrm{R}$-int $=0.084,3770 * \mathrm{~F}>$ $4 * \operatorname{Sig}(\mathrm{F})$, Friedel pairs averaged). Based on a preliminary examination of the crystal, the space group P2(1) was assigned (no exceptions to the systematic absences: H0O, H=odd, 0K0, $\mathrm{K}=$ odd, 00L, L=odd, were noted). The computer programs from the HKL package were used for data reduction.

The preliminary model of the structure was obtained using Xs, a direct methods program. Least-squares refining of the model vs. the data was performed with XL computer program. Illustrations were made with the XP program and tables were made with the XCIF program. All are in the SHELXTL v6.1 package. Thermal ellipsoids shown in the illustrations are at the 50\% level unless otherwise noted.

All non-hydrogen atoms were refined with anisotropic thermal parameters. All $\mathrm{H}$ atoms were constrained with the riding model using the XL program default values.

No further restraints or constraints were imposed on the refinement model. Hydrogen bonding geometries are in Table 8 . 
Table 1. Crystal data and structure refinement for fri61.

Identification code

Empirical formula

Formula weight

Temperature

Wavelength

Crystal system, space group

Unit cell dimensions

Volume

z, Calculated density

Absorption coefficient

$F(000)$

Crystal size

Theta range for data collection

Limiting indices

Reflections collected / unique

Completeness to theta $=27.5$

Absorption correction

Max. and min. transmission

Refinement method

Data / restraints / parameters

Goodness-of-fit on $\mathrm{F}^{\wedge} 2$

Final $R$ indices [ $I>2$ sigma (I)]

$\mathrm{R}$ indices (all data)

Absolute structure parameter

Largest diff. peak and hole fri61

C35 H54 O6

570.78

$150(2) \mathrm{K}$

$0.71073 \mathrm{~A}$

Monoclinic, P 2(1)

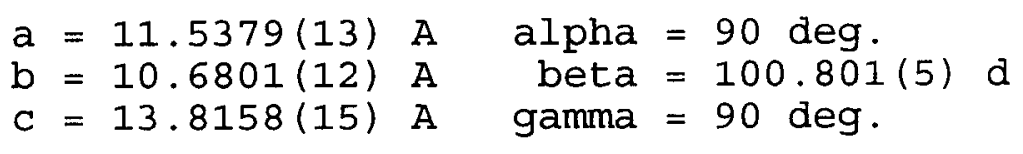

$1672.3(3) \mathrm{A}^{\wedge} 3$

2 , $1.134 \mathrm{Mg} / \mathrm{m}^{\wedge} 3$

$0.075 \mathrm{~mm}^{\wedge}-1$

624

$0.42 \times 0.20 \times 0.17 \mathrm{~mm}$

2.9 to $27.5 \mathrm{deg}$.

$-14<=\mathrm{h}<=14,-13<=\mathrm{k}<=13, \quad-17<=1<=17$

$26655 / 4015[R($ int $)=0.0838]$

$99 \div$

Semi-empirical from equivalents

0.9873 and 0.9690

Full-matrix least-squares on $\mathrm{F}^{\wedge} 2$

$4015 / 1 / 370$

1.078

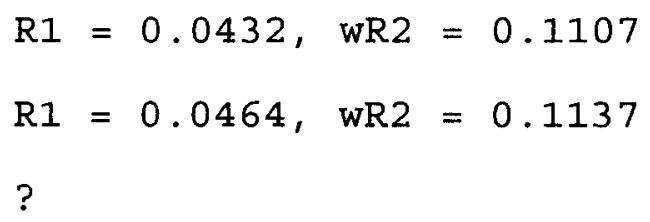

0.278 and -0.208 e. $A^{\wedge}-3$ 